\title{
Evolutionary pattern of karyotypes and meiosis in pholcid spiders (Araneae: Pholcidae): implications for reconstructing chromosome evolution of araneomorph spiders
}

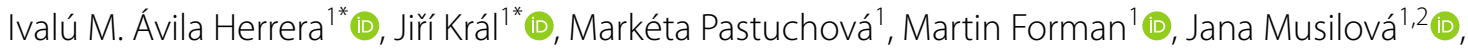

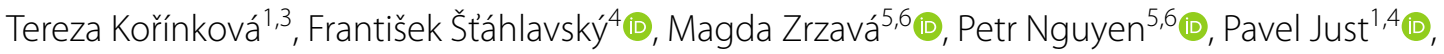 \\ Charles R. Haddad ${ }^{7}$, Matyáš Hiřman ${ }^{4}$, Martina Koubová ${ }^{10}$, David Sadílek ${ }^{1,4}$ (B) and Bernhard A. Huber ${ }^{8}$ (1)
}

\begin{abstract}
Background: Despite progress in genomic analysis of spiders, their chromosome evolution is not satisfactorily understood. Most information on spider chromosomes concerns the most diversified clade, entelegyne araneomorphs. Other clades are far less studied. Our study focused on haplogyne araneomorphs, which are remarkable for their unusual sex chromosome systems and for the co-evolution of sex chromosomes and nucleolus organizer regions (NORs); some haplogynes exhibit holokinetic chromosomes. To trace the karyotype evolution of haplogynes on the family level, we analysed the number and morphology of chromosomes, sex chromosomes, NORs, and meiosis in pholcids, which are among the most diverse haplogyne families. The evolution of spider NORs is largely unknown.

Results: Our study is based on an extensive set of species representing all major pholcid clades. Pholcids exhibit a low $2 \mathrm{n}$ and predominance of biarmed chromosomes, which are typical haplogyne features. Sex chromosomes and NOR patterns of pholcids are diversified. We revealed six sex chromosome systems in pholcids $\left(X 0, X Y, X_{1} X_{2} O, X_{1} X_{2} X_{3} O\right.$, $X_{1} X_{2} Y$, and $X_{1} X_{2} X_{3} X_{4} Y$ ). The number of NOR loci ranges from one to nine. In some clades, NORs are also found on seX chromosomes.

Conclusions: The evolution of cytogenetic characters was largely derived from character mapping on a recently published molecular phylogeny of the family. Based on an extensive set of species and mapping of their characters, numerous conclusions regarding the karyotype evolution of pholcids and spiders can be drawn. Our results suggest frequent autosome-autosome and autosome-sex chromosome rearrangements during pholcid evolution. Such events have previously been attributed to the reproductive isolation of species. The peculiar $X_{1} X_{2} Y$ system is probably ancestral for haplogynes. Chromosomes of the $X_{1} X_{2} Y$ system differ considerably in their pattern of evolution. In some pholcid clades, the $X_{1} X_{2} Y$ system has transformed into the $X_{1} X_{2} 0$ or $X Y$ systems, and subsequently into the $X 0$ system. The $X_{1} X_{2} X_{3} 0$ system of Smeringopus pallidus probably arose from the $X_{1} X_{2} 0$ system by an $X$ chromosome fission. The
\end{abstract}

*Correspondence: avilai@natur.cuni.cz; spider@natur.cuni.cz

${ }^{1}$ Laboratory of Arachnid Cytogenetics, Department of Genetics

and Microbiology, Faculty of Science, Charles University, Viničná 5, 128

44 Prague 2, Czech Republic

Full list of author information is available at the end of the article

(c) The Author(s) 2021, corrected publication 2021. Open Access This article is licensed under a Creative Commons Attribution 4.0 International License, which permits use, sharing, adaptation, distribution and reproduction in any medium or format, as long as you give appropriate credit to the original author(s) and the source, provide a link to the Creative Commons licence, and indicate if changes were made. The images or other third party material in this article are included in the article's Creative Commons licence, unless indicated otherwise in a credit line to the material. If material is not included in the article's Creative Commons licence and your intended use is not permitted by statutory regulation or exceeds the permitted use, you will need to obtain permission directly from the copyright holder. To view a copy of this licence, visit http://creativecommons.org/licenses/by/4.0/. The Creative Commons Public Domain Dedication waiver (http://creativecommons.org/publicdomain/zero/1.0/) applies to the data made available in this article, unless otherwise stated in a credit line to the data. 
$\mathrm{X}_{1} \mathrm{X}_{2} \mathrm{X}_{3} \mathrm{X}_{4} \mathrm{Y}$ system of Kambiwa probably evolved from the $\mathrm{X}_{1} \mathrm{X}_{2} \mathrm{Y}$ system by integration of a chromosome pair. Nucleolus organizer regions have frequently expanded on sex chromosomes, most probably by ectopic recombination. Our data suggest the involvement of sex chromosome-linked NORs in achiasmatic pairing.

Keywords: Achiasmatic pairing, Diffuse stage, Entelegyne, Haplogyne, Inactivation, rDNA, Rearrangement, Segregation, Y chromosome

\section{Background}

The structure of chromosomes changes during evolution, just like any other genomic character. Fine-scale changes of the genome can be easily determined by techniques of DNA sequencing and assembling of sequences. However, long assemblies have gaps containing unknown sequences, which makes it difficult to perform linkage mapping of entire chromosomes. An important tool to complete genome assembly and understand transmission of specific loci are cytogenetic data [1]. Cytogenetics also brings other data on the genome and its dynamics, which are not easy to get by sequencing (e.g. mapping of highly differentiated sex chromosomes) [2, 3] or cannot be obtained by this approach (e.g. chromosome behaviour during nuclear division, or large-scale heterochromatin pattern).

Cytogenetic information is particularly important in the analysis of complex genomes, for example in spiders. Genome evolution of spider ancestors included a polyploid event [4], which might have been the origin of the unusual and complex spider sex chromosome determination. The male sex chromosome complement of most spiders includes several chromosomes that do not recombine during meiosis and are presumably nonhomologous. Furthermore, it probably also contains a chromosome pair formed by the chromosomes X and Y, which recombine and show a very low level of differentiation (further cryptic sex chromosome pair, CSCP) [5]. Some mygalomorph spiders even exhibit two CSCPs [6]. The single CSCP could represent the ancestral sex chromosomes of spiders [7].

Despite recent progress in genomic analysis of spiders [8], their cytogenetics is not satisfactorily understood. Most data concern entelegyne araneomorphs. Although entelegynes exihibit an enormous species diversity (currently nearly 38,700 described species), their karyotypes are usually conservative, comprising a low number $\left(2 \mathrm{n} \mathrm{o}^{-}=10-49\right)$ of exclusively monoarmed (i.e. acrocentric and subtelocentric) chromosomes and containing two different $\mathrm{X}$ chromosomes $\left(\delta^{*} \mathrm{X}_{1} \mathrm{X}_{2} / q \mathrm{X}_{1} \mathrm{X}_{1} \mathrm{X}_{2} \mathrm{X}_{2}\right.$, the socalled $X_{1} X_{2} 0$ system) [9]. The origin of these sex chromosomes is unresolved. The cytogenetics of the other spider clades (mesotheles, mygalomorphs, haplogyne araneomorphs) is far less studied.
Recent phylogenomic analyses of spiders led to considerable changes in the taxonomic composition of haplogyne araneomorphs. Currently, haplogynes consist of the clade Synspermiata (18 families) and a clade formed by the families Hypochilidae and Filistatidae [10-12] (Fig. 1). These two clades currently include more than 6000 described species (based on the data of [13]). Although haplogynes have a much lower species diversity than entelegynes, their karyotypes are very diverse. The male diploid numbers of haplogynes vary from 5 to 152 [14]. According to chromosome structure, haplogynes show two principal patterns. A synapomorphy of the superfamily Dysderoidea is the holokinetic structure of chromosomes. Holokinetic chromosomes do not contain a localised centromere [14]. Karyotypes of all other studied clades are composed of standard (i.e. monokinetic) chromosomes, and are usually predominated by biarmed (i.e. metacentric and submetacentric) chromosomes [9]. Karyotypes of many haplogynes contain the peculiar $\mathrm{X}_{1} \mathrm{X}_{2} \mathrm{Y}$ system $\left(\mathrm{o}_{1} \mathrm{X}_{2} \mathrm{Y} /\right.$ \% $\left.\mathrm{X}_{1} \mathrm{X}_{1} \mathrm{X}_{2} \mathrm{X}_{2}\right)$, a sex chromosome constitution that has been found in seven families $[9,14$ 17]. Chromosomes of the $X_{1} X_{2} Y$ system usually exhibit a metacentric morphology. They pair without chiasmata and recombination (i.e. achiasmatically) during male meiosis. The phylogenetic distribution of the $\mathrm{X}_{1} \mathrm{X}_{2} \mathrm{Y}$ system suggests a considerable antiquity of this sex chromosome determination [9]. According to a recent hypothesis, this system could be ancestral for araneomorph spiders [16]. Despite this, the origin and subsequent evolutionary transformations of the $\mathrm{X}_{1} \mathrm{X}_{2} \mathrm{Y}$ system are not satisfactorily understood. Haplogynes also exhibit other unusual cytogenetic traits, namely a considerable decondensation of bivalents during a specific period of male prophase I (so-called diffuse stage) $[9,18]$ and the frequent occurrence of nucleolus organizer regions (NORs) on the sex chromosomes [9]. Nucleolus organizer regions are chromosome regions crucial for the formation of the nucleolus. They contain tandem copies of the genes coding 5.8S, $18 \mathrm{~S}$, and $28 \mathrm{~S}$ ribosomal RNA [19].

Although fundamental trends of haplogyne karyotype evolution have been determined [9], karyotype evolution within haplogyne families remains unknown. To fill this gap, we have focused on the cytogenetics of the family Pholcidae (Fig. 1), which is an ideal model group for such a study. First, pholcids are the most diversified haplogyne 


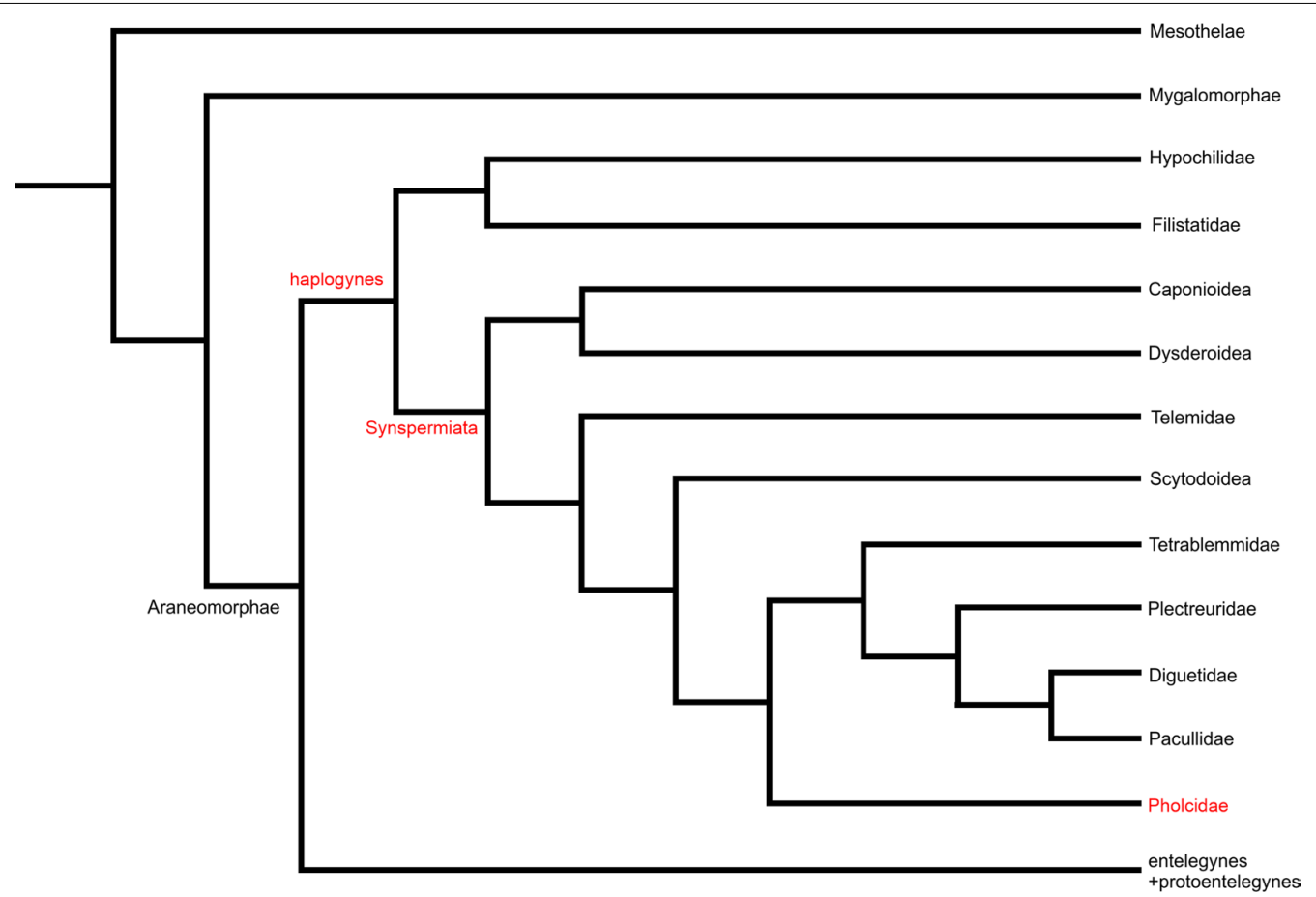

Fig. 1 Phylogeny of spiders, with specific consideration on haplogyne spiders. The phylogenetic tree was constructed following [10] except for Telemidae [1 1]. Caponioidea: Caponiidae, Trogloraptoridae; Dysderoidea: Dysderidae, Oonopidae, Orsolobidae, Segestriidae; Scytodoidea: Drymusidae, Ochyroceratidae, Periegopidae, Psilodercidae, Scytodidae, Sicariidae

family with a standard chromosome structure. They currently include 94 genera and 1812 species [13]. Second, pholcids are the spider family with the most comprehensive molecular phylogeny available (with 600 species representing $86 \%$ of the known genera [20, 21]). Third, they display a worldwide distribution, with the large majority of species in the tropics and subtropics. Numerous species are synanthropic and have been translocated by humans around the globe [22, 23].

Information on pholcid cytogenetics is very limited. There are only basic data available for 23 species representing nine genera (see database [24]). Here we present chromosome data of 47 species, which represent an extensive cross-section through all major pholcid clades. Since previous authors often failed in the proper determination of pholcid cytogenetic data, we have also revised previously published results. We further focused on the evolution of NORs, the evolution of which is largely unknown in spiders.

Our study represents one of the most comprehensive cytogenetic datasets of any spider family. Cytogenetic data of pholcids are diversified and have considerable potential to be used in the reconstruction of pholcid phylogeny. Based on our data, numerous conclusions and hypotheses regarding the karyotype evolution of pholcids, as well as haplogyne spiders, can be drawn. Finally, our results suggest promising subjects for future evolutionary studies on the karyotypes, sex chromosomes, and NORs of spiders.

\section{Results}

\section{Arteminae}

Our study included representatives of the genera Artema, Chisosa, Holocneminus, Physocyclus, and Wugigarra (Additional file 1: Table S1, Additional file 2: Table S2). The diploid number in artemine males ranged from $2 \mathrm{n}=13$ (Chisosa diluta) to $2 \mathrm{n}=33$ (Artema spp.), with most species having $2 \mathrm{n}=15$. In all species, the karyotypes consisted predominantly of biarmed chromosomes (Additional file 2: Table S2, Fig. 2, Additional file 3: Fig. S1).

Representatives of Artema differed from the other artemines by a much higher diploid number and a $\mathrm{X}_{1} \mathrm{X}_{2} \mathrm{Y}$ system. The male karyotype of Artema contained 15 chromosome pairs (this term is used in a specific context in our study, see "Methods", p. 31) decreasing gradually in size (Additional file 1: Table S1, Additional file 2: Table S2, Additional file 4: Fig. S2). Chromosome morphology was predominantly metacentric. The $\mathrm{X}_{1}$ chromosome was considerably longer than $\mathrm{X}_{2}$. The $\mathrm{Y}$ 


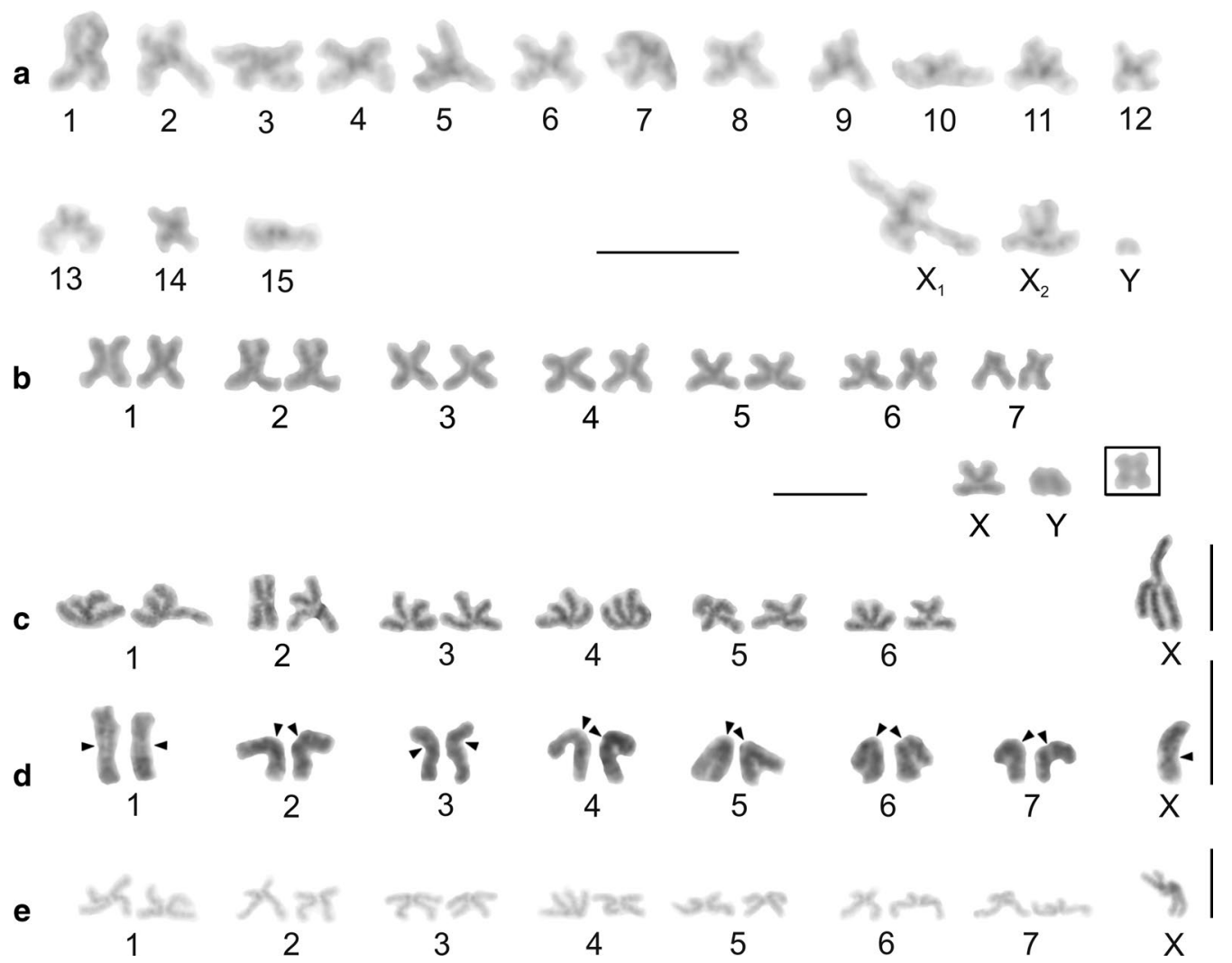

Fig. 2 Arteminae, male karyotypes, stained by Giemsa. Based on two sister metaphases II except for Artema nephilit (single metaphase II) and Holocneminus (spermatogonial metaphase). a Artema nephilit, haploid karyotype. Chromosomes metacentric except for five submetacentric (nos. 2, 8, 10-12), one subtelocentric (no. 13), and one acrocentric element (no. 15). The Y chromosome is from another metaphase II. Morphology of the Y chromosome is unresolved; b Wugigarra sp. Karyotype metacentric except for one subtelocentric pair (no. 7). Inset: magnified Y chromosome (from another metaphase II). Note metacentric morphology of this element; c Chisosa diluta. Chromosomes exclusively metacentric. The positively heteropycnotic X chromosome is the longest chromosome; $\mathbf{d}$ Holocneminus sp. Chromosomes metacentric except for one submetacentric (no. 4) and one subtelocentric pair (no. 3). Centromeres marked by arrowheads; e Physocyclus dugesi. Chromosomes metacentric except for two submetacentric (nos 2, 5) and two subtelocentric pairs (nos 6, 7). The positively heteropycnotic X chromosome is the longest chromosome of the karyotype. Bar $=10 \mu \mathrm{m}$

chromosome was the smallest element of the karyotype (Additional file 1: Table S1, Additional file 2: Table S2, Fig. 2a, Additional file 3: Fig. S1).

Karyotypes of the other artemines contained only six (C. diluta) or seven chromosome pairs (the other species). Chromosome pairs decreased gradually in size except for the prominent longest pair of $C$. diluta (Additional file 1: Table S1). The karyotype of Wugigarra $\left(2 \mathrm{n}^{\star}=16\right)$ was characterized by a $\mathrm{XY}$ system (Fig. 2b, Additional file 4: Fig. S2). The Y chromosome was the smallest element of the complement, a feature similar to Artema (Additional file 1: Table S1). The other taxa exhibited the X0 system (Fig. 2c-e, Additional file 5: Fig. S3). Their sex chromosome was the longest element of the karyotype except for Holocneminus sp. The size of the $\mathrm{X}$ chromosome varied considerably from $11.4 \%$ (Holocneminus sp.) to $21.2 \%$ of
TCL (C. diluta). The length of the sex chromosome was determined from mitotic metaphase (Holocneminus) or metaphase II (the other X0 species). The small size of the $\mathrm{X}$ chromosome in Holocneminus compared to the other species could then reflect the fact that this element is more condensed in mitotic metaphase than in metaphase II.

The nucleolus organizer region was present at an end of one chromosome pair in all of the studied species (Additional file 2: Table S2, Fig. 3, Additional file 6: Fig. S4), and in Physocyclus also on both termini of the $\mathrm{X}$ chromosome (Fig. 3d). The morphology of the NOR-bearing pair was metacentric in Holocneminus (Fig. 3c), metacentric/submetacentric in Physocyclus (Fig. 3d), submetacentric in Artema (Fig. 3a, Additional file 6: Fig. S4a, b), and subtelocentric in Wugigarra 

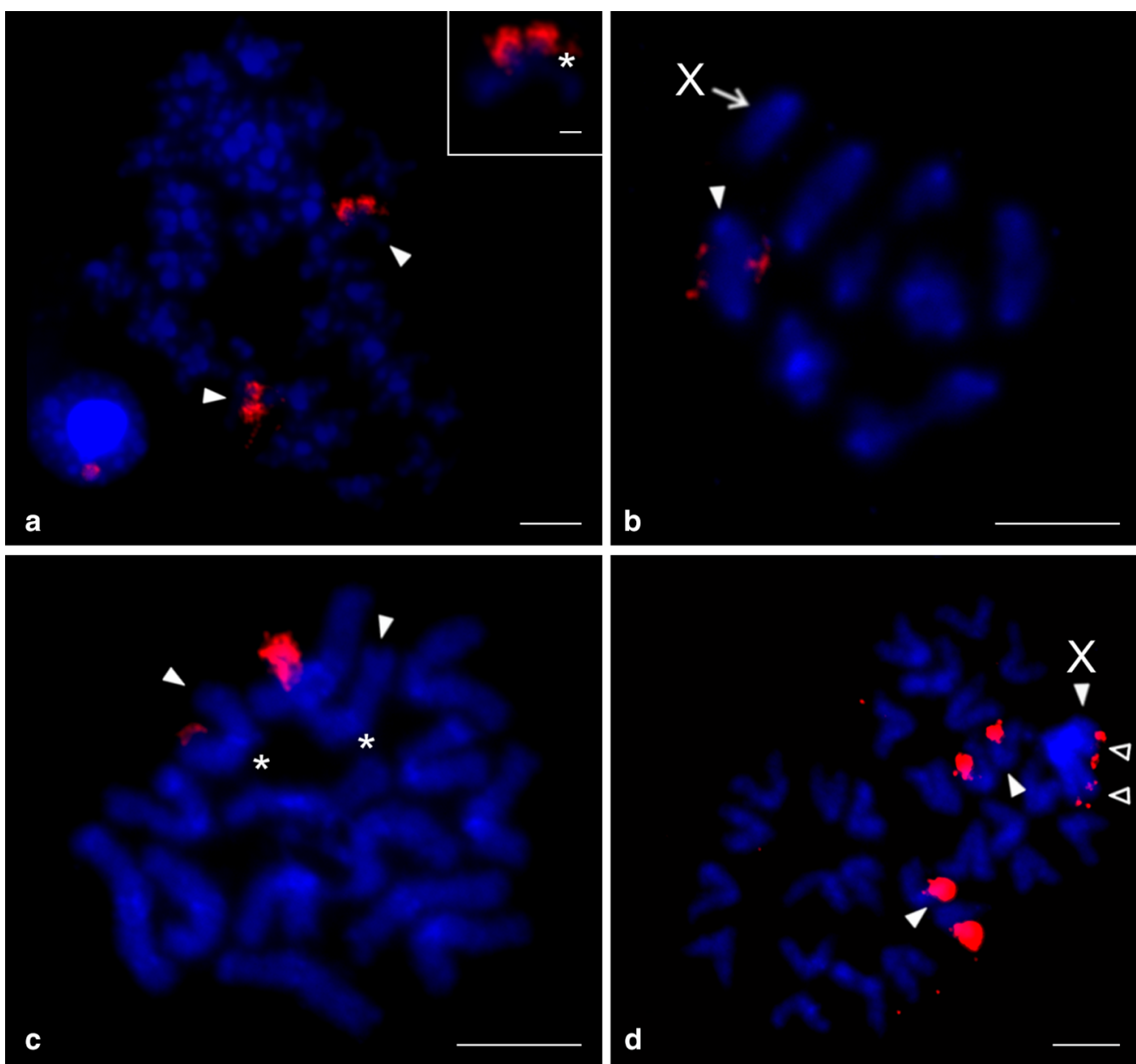

Fig. 3 Arteminae, males, detection of NORs (FISH). Arrowhead=NOR-bearing chromosome (a, $\mathbf{c}$, d) or bivalent (b), asterisk=centromere, open arrowhead $=$ sex chrosomosome-linked NOR, $X=X$ chromosome. $\mathbf{a}$ Artema nephilit $\left(X_{1} X_{2} Y\right)$, plate formed by fused sister metaphases II. Pair of chromosomes bears a terminal NOR. Inset: submetacentric NOR-bearing chromosome. NOR is placed at the end of short arm; $\mathbf{b}, \mathbf{c}$ Holocneminus sp. (X0). b Metaphase I, one bivalent contains NOR; c Mitotic metaphase. Chromosomes of one metacentric pair have terminal NOR; $\mathbf{d}$ Physocyclus dugesi (X0), two sister plates, late metaphase II. Chromosomes of one metacentric/submetacentric pair contain a terminal NOR. X chromosome is more condensed than the other chromosomes. It includes tiny NOR at both ends. Bar $=5 \mu \mathrm{m}$, inset $1 \mu \mathrm{m}$

(Additional file 6: Fig. S4c). Except for Holocneminus, the NOR was placed at the end of the short arm.

\section{Modisiminae}

We analysed representatives of Anopsicus, Modisimus, and Psilochorus (Additional file 7: Table S3). The studied modisimines exhibited the same male diploid number (17), sex chromosome system (SCS) (X0), and a metacentric sex chromosome (Additional file 1: Table S1, Additional file 7: Table S3, Fig. 4a-c, Additional file 8: Fig. S5, Additional file 9: Fig. S6). Chromosomes of modisimines were biarmed except for one pair of P. californiae (Additional file 1: Table S1, Additional file 7: Table S3). The sex chromosome was the longest chromosome of the karyotype (Additional file 1: Table S1).

The genus Psilochorus was represented by three species. Chromosome pairs of $P$. californiae decreased gradually in size. In contrast, the longest pair of $P$. pallidulus and the two longest pairs of $P$. simoni were prominent. Moreover, in the latter two species the last pair was much smaller than the penultimate pair (Additional file 1: Table S1). The karyotype of P. pallidulus was formed exclusively by metacentric chromosomes (Additional file 9: Fig. S6b). The karyotypes of the other two species were also formed by metacentrics except for two submetacentric pairs of P. simoni, and two submetacentric and one subtelocentric pair 


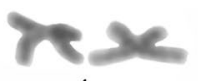

1

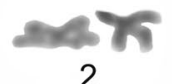

a
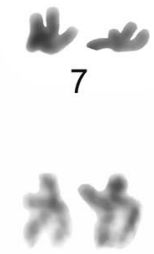

1

b

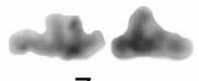

7

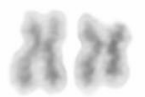

1

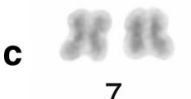

7

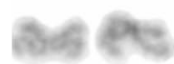

1

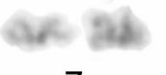

7

d

\section{3}

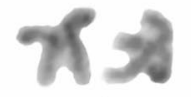

2

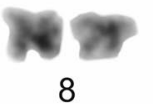

2
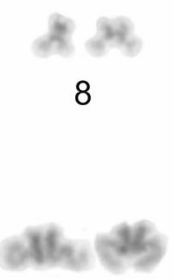

2
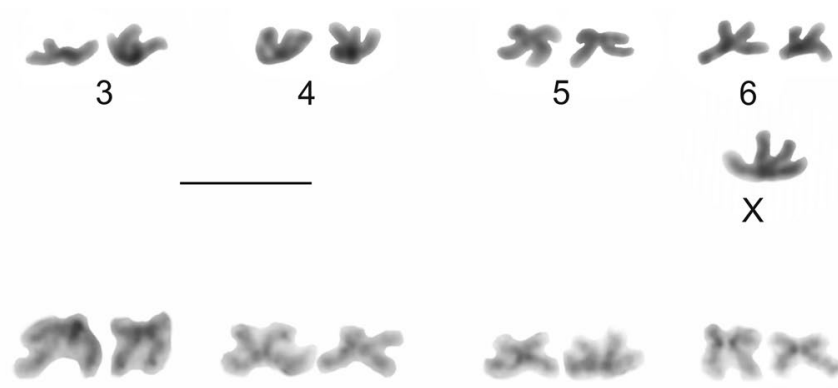

3

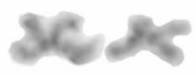

4
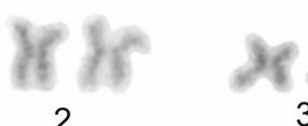

3

4

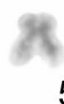

5

6

3

4
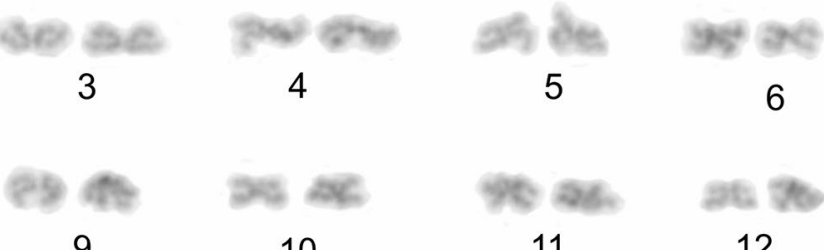

9

10
5
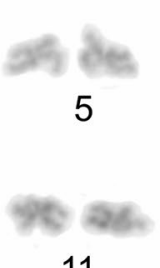

6

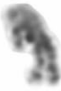

$\mathrm{X}$

Fig. 4 Modisiminae $(\mathbf{a}-\mathbf{c})$ and Ninetinae $(\mathbf{d})$, male karyotypes, stained by Giemsa. Based on two sister metaphases II. Karyotypes are predominated by metacentrics. Single sex chromosome of modisimines is the longest element of the karyotype. a Anopsicus sp., pair nos. 3 and 5 are submetacentric; b Modisimus cf. elongatus. Three pairs submetacentric (nos 1, 3, 6), X chromosome positively heteropycnotic; c Psilochorus simoni. Chromosome pairs form three size groups (pairs 1-2, 3-7, 8). Two pairs submetacentric (nos 1, 5). Sex chromosome negatively heteropycnotic; $\mathbf{d}$ Pholcophora americana, pair no. 2 submetacentric. Bar $=10 \mu \mathrm{m}$

of P. californiae (Fig. 4c, Additional file 9: Fig. S6a). Nucleolus organizer regions were detected in P. californiae and $P$. pallidulus. Their karyotype included a NOR-bearing metacentric pair. While the NOR of $P$. californiae exhibited a terminal location, placement of NOR in P. pallidulus was not determined. In both species, the sex chromosome bore NORs at both ends (Fig. 5).

\section{Ninetinae}

We studied representatives of Kambiwa and Pholcophora (Additional file 7: Table S3, Additional file 10: Fig. S7). The chromosomes of $K$. neotropica were metacentric except for two monoarmed elements (Additional file 11: Fig. S8a). Several lines of observations demonstrated a complicated sex chromosome system in Kambiwa. Sex chromosomes formed a peculiar cross-shaped element in 


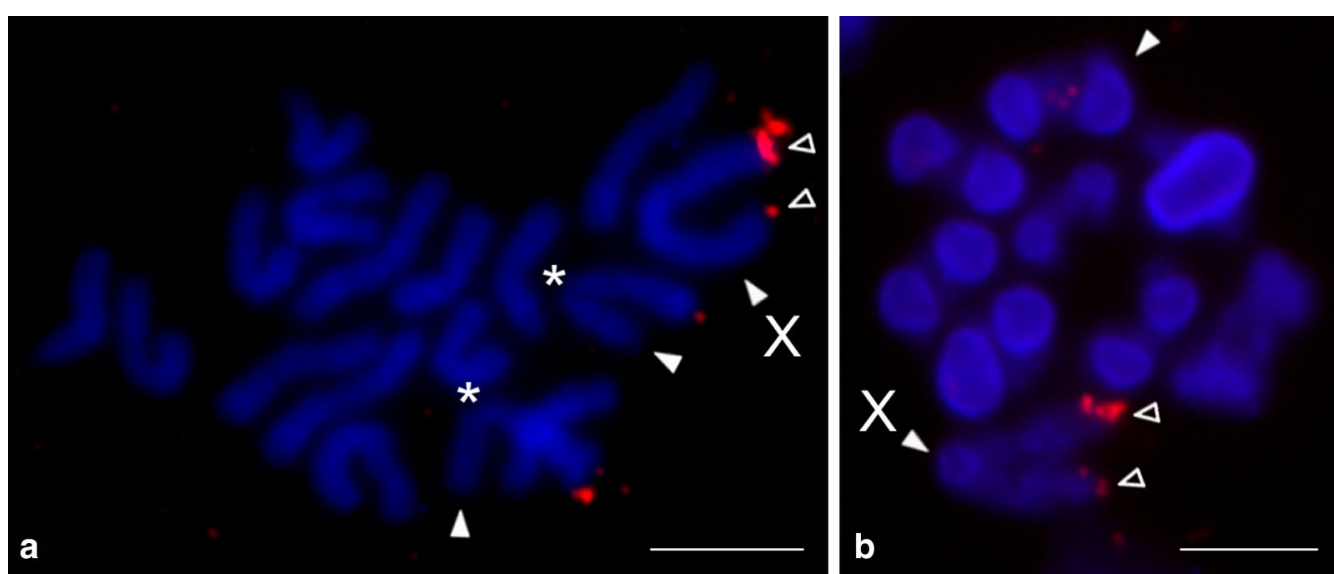

Fig. 5 Modisiminae, males, detection of NORs (FISH). Arrowhead = NOR bearing chromosome (a) or bivalent (b), asterisk=centromere, open arrowhead $=$ sex chromosome-linked NOR, $X=X$ chromosome. a Psilochorus californiae (X0), mitotic metaphase. Chromosomes of one metacentric pair contain a terminal NOR. X chromosome bears NOR at both ends; $\boldsymbol{b}$ P. pallidulus (X0), metaphase I. One bivalent includes a tiny NOR. X chromosome has two terminal NORs. Bar $=5 \mu \mathrm{m}$

male metaphase I (Additional file 10: Fig. S7a, b). Comparison of data on male $2 n(2 n \hat{\imath}=29$, Additional file 11: Fig. S8a) and male metaphase I (12 bivalents plus crossshaped element) (Additional file 10: Fig. S7a, b) suggested that the cross-shaped element is a multivalent comprising of five chromosomes. Analysis of male metaphases II demonstrated the presence of a $\mathrm{X}_{1} \mathrm{X}_{2} \mathrm{X}_{3} \mathrm{X}_{4} \mathrm{Y}$ system. There are two types of metaphase II plates, one with 13 chromosomes including the Y microchromosome, and another one with 16 chromosomes including four heterochromatic X (Fig. 6b, Additional file 11: Fig. S8b, c). Two $\mathrm{X}$ chromosomes differed from the remaining two by a specific condensation at metaphase II. These chromosomes were monoarmed (Figs. 6b). The morphology of the tiny $\mathrm{Y}$ chromosome was unresolved. Chromosomes of Pholcophora americana $\left(2 \mathrm{n} \mathrm{O}^{*}=29, \mathrm{X}_{1} \mathrm{X}_{2} \mathrm{Y}\right)$ decreased gradually in size except for the prominent longest pair (Additional file 1: Table S1). They were metacentric
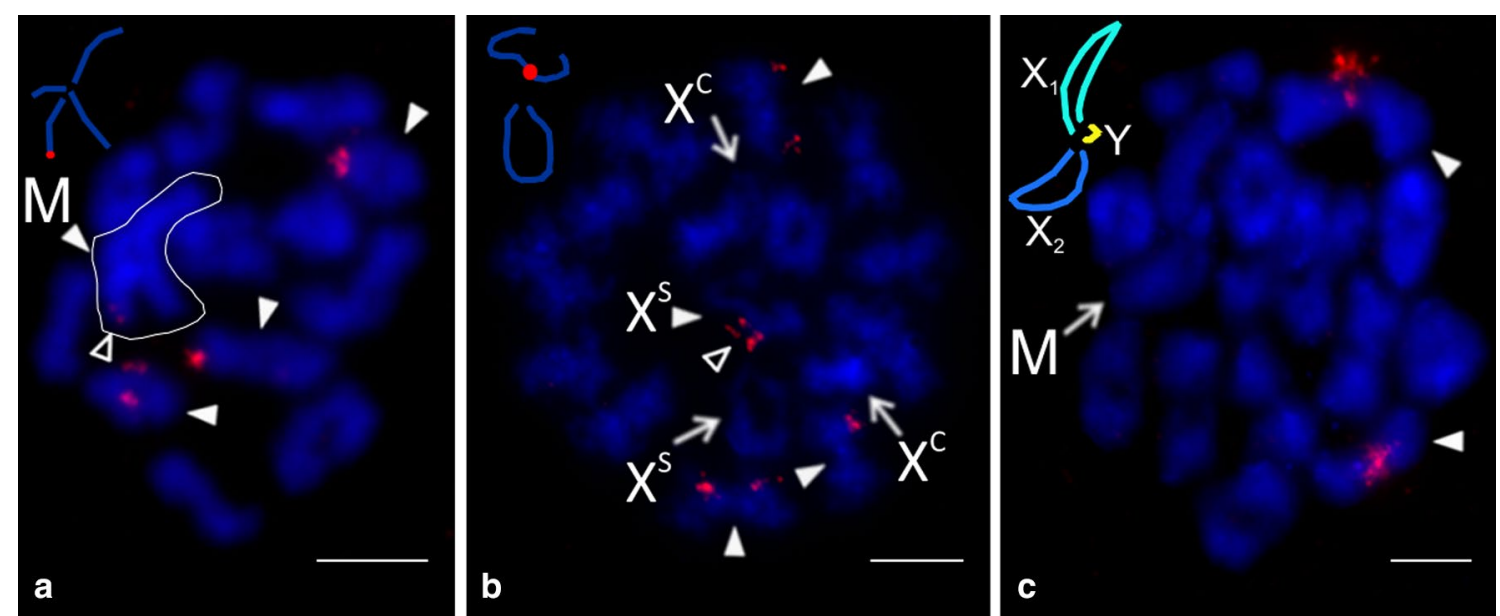

Fig. 6 Ninetinae, males, detection of NORs (FISH). Figures contain scheme of sex chromosomes (red=NOR). Arrowhead=chromosome (b), bivalent $(\mathbf{a}, \mathbf{c})$ or multivalent $(\mathbf{a})$ with NOR, $\mathrm{M}=$ sex chromosome multivalent, open arrowhead = sex chromosome-linked NOR, $X=X$ chromosome. a, $\mathbf{b}$ Kambiwa neotropica $\left(\mathrm{X}_{1} \mathrm{X}_{2} \mathrm{X}_{3} \mathrm{X}_{4} \mathrm{Y}\right)$ a Metaphase I, three bivalents contain terminal NOR. Moreover, sex chromosome multivalent (delimited by line) includes a tiny NOR at end of one "arm". Note scheme for morphology of multivalent. b Metaphase II $(n=16)$ containing four $X$ chromosomes. Two $X$ chromosomes are the most condensed elements of the plate $\left(X^{C}\right)$. Two other $X$ chromosomes have lower condensation and monoarmed morphology $\left(X^{S}\right)$. Note scheme for morphology of monoarmed $X$ chromosomes. One monoarmed $X$ contains a NOR at short arm. In addition, three other chromosomes bear a NOR; $\mathbf{c}$ Pholcophora americana $\left(X_{1} X_{2} Y\right)$, metaphase I. Two large bivalents contain terminal NOR. Note scheme for probable morphology of sex chromosome multivalent. Arms of each $X$ chromosome are associated with each other. Bar $=5 \mu m$ 
except for one submetacentric pair (Fig. 4d). The X chromosomes showed a similar length. While they were the longest elements of the karyotype, the Y chromosome was the smallest one (Additional file 1: Table S1).

Three chromosome pairs of Kambiwa contained a terminal NOR (Fig. 6a). Moreover, one monoarmed X chromosome bore a NOR on its short arm (Fig. 6b). Pholcophora exhibited two chromosome pairs bearing terminal NORs (Fig. 6c).

\section{Pholcinae}

We analysed pholcines representing twelve genera (Additional file 12: Table S4). Male $2 \mathrm{n}$ ranged from 9 (Micropholcus fauroti) to 25 (Nipisa deelemanae, three Pholcus species, Spermophora senoculata) (Additional file 12: Table S4). Chromosome morphology was determined in representatives of eight genera (Additional file 1: Table S1). Chromosome pairs of most pholcines decreased gradually in size. In several species, however, the longest pair(s) were prominent (first pair of Belisana sabah and Pholcus opilionoides; first three pairs of Leptopholcus guineensis; first five pairs in Pholcus sp.). In Aetana kinabalu the last chromosome pair was much smaller than the penultimate pair (Additional file 1: Table S1). Chromosome pairs were biarmed, except for two subtelocentric pairs in Quamtana filmeri, one subtelocentric pair in Pholcus kindia, and one acrocentric pair in A. kinabalu and Pholcus sp. (Additional file 12: Table S4).

Concerning sex chromosomes, most pholcines showed the $\mathrm{X}_{1} \mathrm{X}_{2} \mathrm{Y}$ system, which was found in nine genera (Aetana, Leptopholcus, Metagonia, Muruta, Nipisa, Pehrforsskalia, Pholcus, Quamtana, Spermophora) (Additional file 1: Table S1, Fig. 7a-d, Additional file 13: Fig. S9, Additional file 14: Fig. S10, Additional file 15: Fig. S11, Additional file 16: Fig. S12). The $\mathrm{X}_{1}$ chromosome was usually the largest element of the karyotype, the $\mathrm{X}_{2}$ chromosome a small to medium element, and the $\mathrm{Y}$ chromosome usually a microchromosome or small element (Additional file 1: Table S1). While the $X_{1}$ and $X_{2}$ chromosomes of Aetana and Spermophora were medium-sized elements of similar size, the $Y$ chromosome of these species was a microchromosome (Additional file 1: Table S1). Although there are no precise data on the morphology of the sex chromosomes in Metagonia, they probably also consist of $\mathrm{X}$ chromosomes of similar size and a tiny $\mathrm{Y}$ chromosome (Additional file 11: Fig. S8f, Additional file 14: Fig. S10e). Most other pholcines exhibiting the $\mathrm{X}_{1} \mathrm{X}_{2} \mathrm{Y}$ system showed a $\mathrm{X}_{2}$ size reduction (up to $2.7 \%$ of TCL in Pholcus pagbilao) and a considerable increase of the Y chromosome size (up to $11.7 \%$ of TCL in P. kindia). In some Pholcus and Quamtana species, the $\mathrm{X}_{2}$ chromosome was only slightly larger than the $\mathrm{Y}$ chromosome (Additional file 1: Table S1). In Leptopholcus and two Pholcus species (P. kindia, P. pagbilao), the $\mathrm{Y}$ chromosome was even longer than the $\mathrm{X}_{2}$ chromosome (Additional file 1: Table S1, Fig. 7a, b, Additional file 16: Fig. S12b). The $\mathrm{X}_{1}$ chromosome of pholcines was metacentric, except for Aetana, whose $\mathrm{X}_{1}$ chromosome was submetacentric (Additional file 12: Table S4, Additional file 16: Fig. S12a). In contrast, the morphology of the $\mathrm{X}_{2}$ was variable. It was metacentric in Aetana, Nipisa, Spermophora, and African Pholcus species (Fig. 7b, Additional file 11: Fig. S8i, Additional file 12: Table S4, Additional file 13: Fig. S9f, Additional file 16: Fig. S12a), submetacentric in Leptopholcus, Muruta, and Pholcus phalangioides (Additional file 12: Table S4, Fig. 7a, Additional file 15: Fig. S11b), subtelocentric in Quamtana filmeri and Pholcus sp. (Fig. 7c, d, Additional file 12: Table S4), and acrocentric in Quamtana hectori, Pholcus opilionoides and P. pagbilao (Additional file 12: Table S4, Additional file 16: Fig. S12b-d). The Y chromosome exhibited a metacentric morphology, except for Leptopholcus and P. pagbilao, whose Y chromosome was submetacentric (Fig. 7a, Additional file 12: Table S4, Additional file 16: Fig. S12b). The morphology of the Y chromosome of Aetana, Muruta, and Nipisa, as well as sex chromosomes of Metagonia and Pehrforsskalia, were not determined.

Some pholcines displayed the X0 system (Belisana, Cantikus, and Micropholcus). The X chromosome was metacentric (Additional file 12: Table S4, Figs. 7e, f, 8a, b, Additional file 17: Fig. S13). Its size was comparable to the size of short (Micropholcus) or medium-sized chromosome pairs (Belisana, Cantikus) (Additional file 1: Table S1).

Nucleolus organizer regions were detected in representatives of nine genera, namely Aetana, Belisana, Cantikus, Micropholcus, Muruta, Nipisa, Pholcus, Quamtana, and Spermophora (Additional file 12: Table S4). Pholcines showed a considerable diversity of NOR patterns. Their karyotypes contained from one (Cantikus, Micropholcus, Muruta, Q. filmeri) to five NOR-bearing chromosome pairs (Belisana) (Figs. 8b, 9, 10, Additional file 18: Fig. S14). In most species, we also determined the morphology of these pairs, including the NOR location. Pairs bearing NORs were biarmed; NORs were placed at chromosome ends (Additional file 12: Table S4). All pairs included one NOR locus except for one pair of Nipisa, P. pagbilao, and Spermophora, which had a locus at both ends (Figs. 9a, 10c, Additional file 18: Fig. S14b). In Spermophora, both loci of this pair were homozygous for the presence of a NOR (Fig. 9a). In the studied male of $P$. pagbilao, one of these loci was heterozygous for the presence of a NOR (Fig. 10c). Concerning the studied Nipisa male, comparison of the NOR pattern 


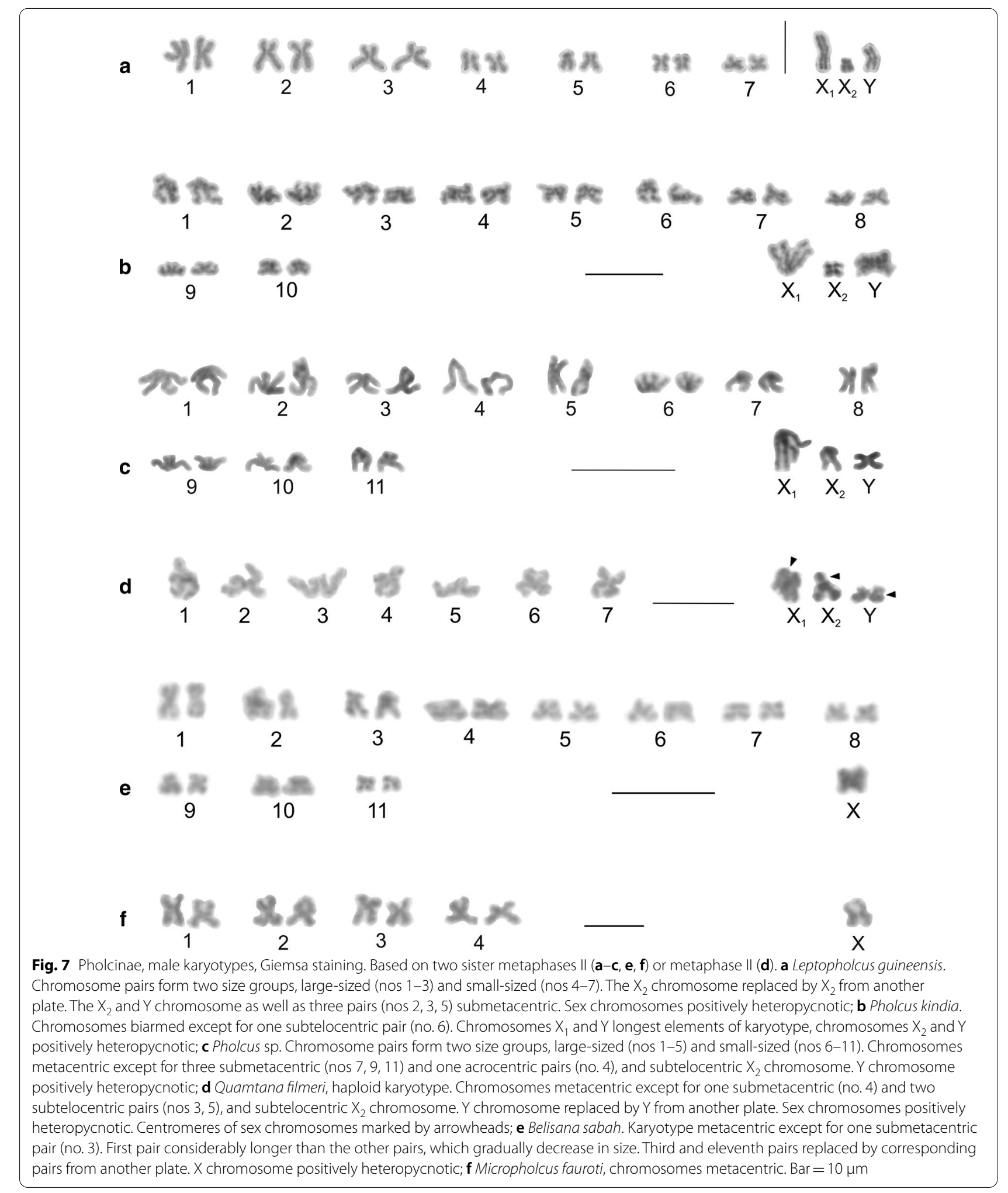



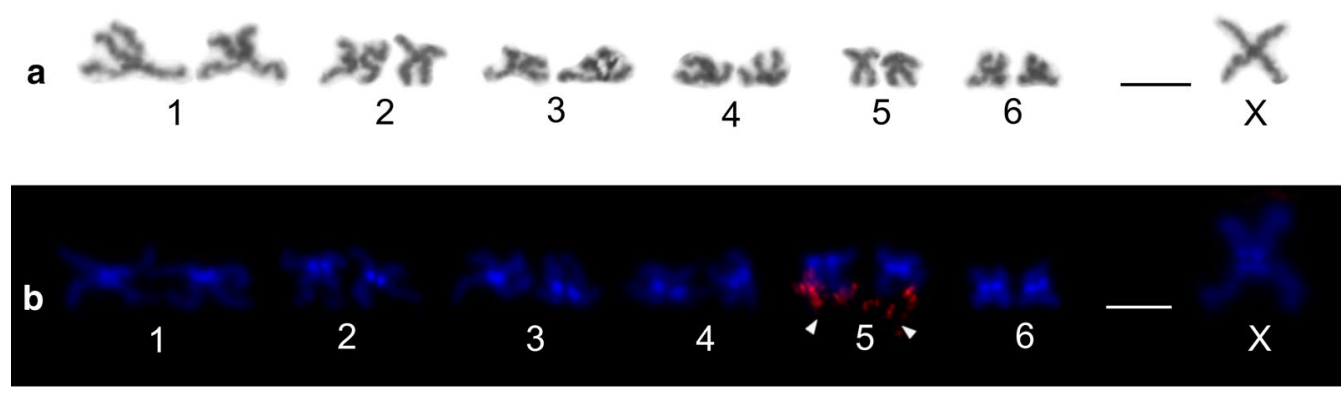

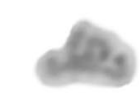

1

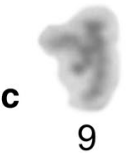

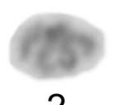

2

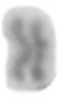

10

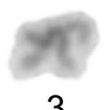

3

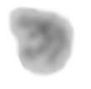

11

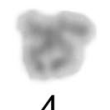

4

5

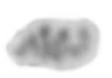

12

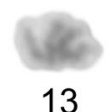

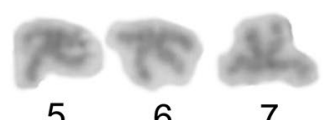

6
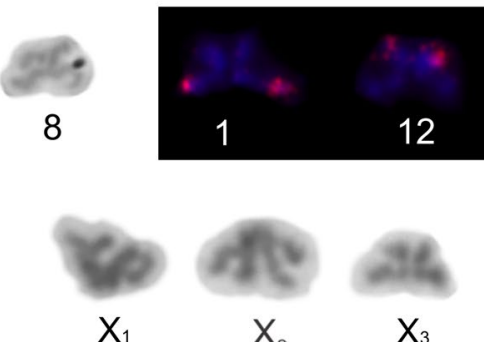

Fig. 8 Male karyotype of Cantikus sabah (Pholcinae) $(\mathbf{a}, \mathbf{b})$ and Smeringopus pallidus (Smeringopinae) (c). Based on metaphase II (c) or two sister metaphases II (a, b). Arrowhead = NOR. a Diploid karyotype, Giemsa stained. Note metacentric morphology of chromosomes. b Diploid karyotype, detection of NORs (FISH). Fifth pair bears a terminal NOR (arrowheads). In contrast to most other pholcids, centromeres are formed by a prominent AT-rich block; c Haploid karyotype, Giemsa stained. Chromosomes metacentric except for three submetacentrics (nos 5, 9, 12). Sex chromosomes show a slight positive heteropycnosis. Inset: detection of NORs (FISH); chromosomes of two pairs (nos. 1, 12) contain terminal NOR. Bar $=10 \mu m$

of the chromosome pairs in metaphase I (three NOR bearing pairs, Additional file 18: Fig. S14c) and mitotic metaphase (five chromosomes with NOR, including one chromosome with NOR at both ends, Additional file 18: Fig. S14b) implied that one chromosome pair consists of a chromosome with NOR at both ends plus a chromosome with one NOR or without NOR. It means that one or even both loci of this pair were heterozygous for the presence of a NOR.

Sex chromosome-linked NORs were revealed in Aetana, Muruta, Nipisa, Quamtana, Pholcus phalangioides $\left(\mathrm{X}_{1} \mathrm{X}_{2} \mathrm{Y}\right)$, and Belisana (X0) (Additional file 12: Table S4, Figs. 9b-f, 10a, b, Additional file 18: Fig. S14b-h). In Aetana, the $\mathrm{X}_{1}$ chromosome had a terminal NOR (Fig. 9b). In Muruta, Nipisa, Quamtana, and P. phalangioides, NORs were detected at both ends of the $X_{1}$ chromosomes (Additional file 12: Table S4, Figs. 9f, 10b, Additional file 18: Fig. S14b, d, f-h). Furthermore, the $\mathrm{X}_{2}$ chromosome of some pholcines involved NOR(s) too. While the $\mathrm{X}_{2}$ chromosome of Muruta and P. phalangioides exhibited NOR at one end only (Figs. 9f, 10b), the $\mathrm{X}_{2}$ chromosome of Nipisa was terminated by NOR at both ends (Additional file 18: Fig. S14b). In the latter pholcid, the $\mathrm{Y}$ chromosome also had a terminal NOR (Additional file 18: Fig. S14b). In Belisana, a NOR was detected at both ends of the $\mathrm{X}$ chromosome (Fig. 9c, d). In species exhibiting the $\mathrm{X}_{1} \mathrm{X}_{2} \mathrm{Y}$ system, the sex chromosome ends bearing a NOR took part in sex chromosome pairing during male meiosis (Figs. 9b, e, 10a, Additional file 18: Fig. S14c, e).

\section{Smeringopinae}

We analysed representatives of Crossopriza, Holocnemus, Hoplopholcus, Smeringopus, and Stygopholcus (Additional file 19: Table S5). The male $2 n$ ranged from 23 to 29 (Additional file 19: Table S5, Figs. 8c, 11, Additional file 20: Fig. S15, Additional file 21: Fig. S16). Chromosome pairs of most smeringopines decreased gradually in length. In some species the longest pair (Holocnemus, Smeringopus cylindrogaster) or two longest pairs (Crossopriza lyoni, Stygopholcus skotophilus) were prominent. Furthermore, the last pair of S. skotophilus was much smaller than the penultimate pair. Chromosome pairs were exclusively biarmed except for Holocnemus hispanicus, S. skotophilus, and some Hoplopholcus and Smeringopus species, whose karyotype included one monoarmed pair (Additional file 1: Table S1).

The male karyotype of Hoplopholcus and Smeringopus was composed of 28 chromosomes, including the $\mathrm{X}_{1} \mathrm{X}_{2} 0$ system (Additional file 19: Table S5, Fig. 11d, f, Additional file 20: Fig. S15, Additional file 22: Fig. S17a-e), except for S. pallidus $\left(2 \mathrm{n} \sigma^{\star}=29, \mathrm{X}_{1} \mathrm{X}_{2} \mathrm{X}_{3} 0\right)$ (Fig. 8c, Additional 

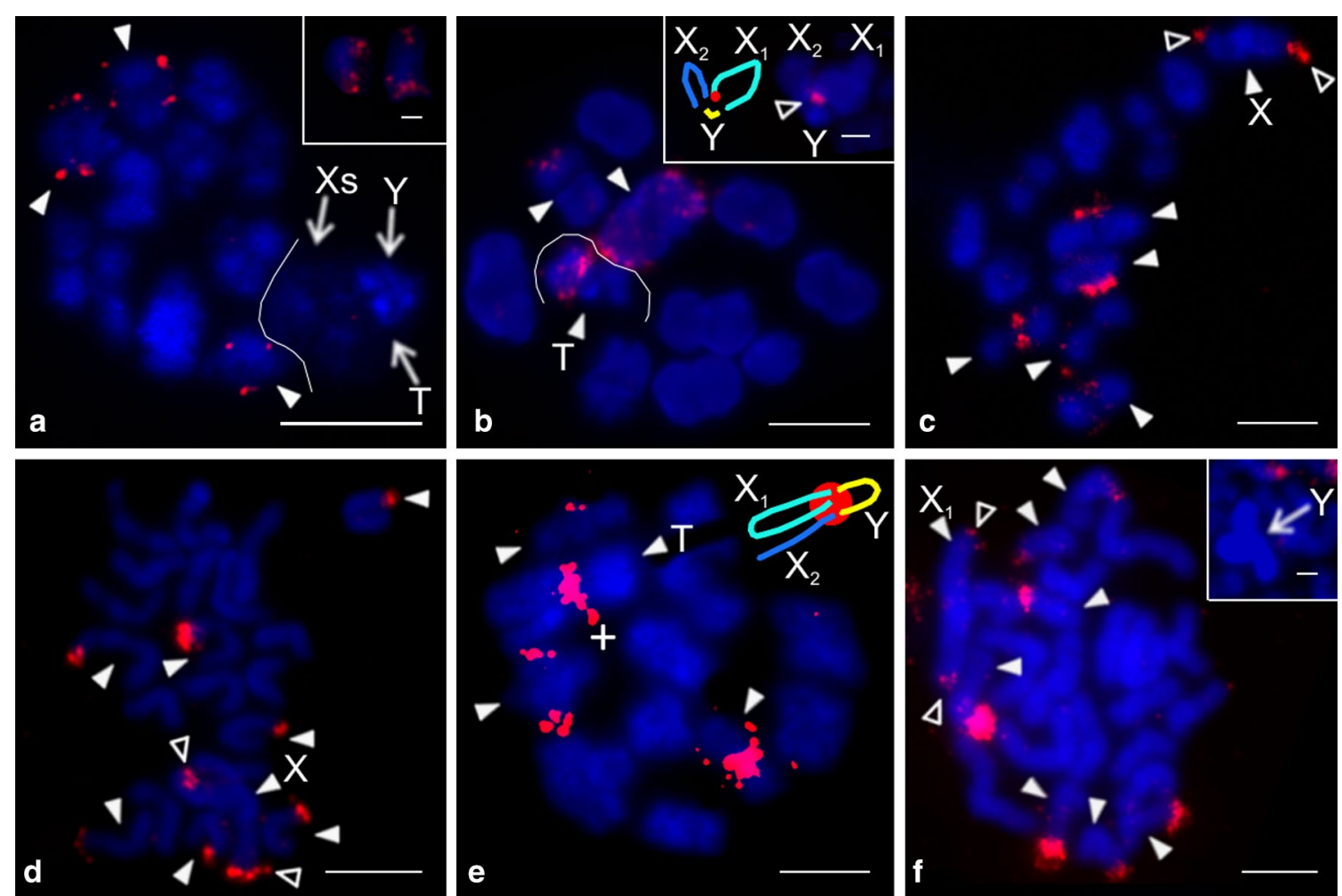

Fig. 9 Pholcinae, males, NORs (FISH) I. Figures $\mathbf{b}$, e contain scheme of sex chromosome trivalent $X_{1} X_{2} Y($ red circle at $\mathbf{c}=$ signal formed by several NORs). Arrowhead = NOR bearing chromosome $(\mathbf{c}, \mathbf{d}, \mathbf{f})$, bivalent $(\mathbf{a}-\mathbf{c}, \mathbf{e})$ or trivalent $(\mathbf{b}, \mathbf{e})$, open arrowhead = sex chromosome-linked NOR, $T=$ sex chromosome trivalent $(\mathbf{a}, \mathbf{b}, \mathbf{e}), X=X$ chromosome, $X_{1}=X_{1}$ chromosome, $X_{2}=X_{2}$ chromosome, $X s=X$ chromosomes, $Y=Y$ chromosome, $+=$ signal formed by several NORs. a Spermophora senoculata $\left(X_{1} X_{2} Y\right)$, diakinesis, note three NOR-bearing bivalents, sex chromosome trivalent separated by line (X chromosomes considerably decondensed). Inset: mitotic metaphase, chromosome pair bearing two terminal NOR loci; b Aetana kinabalu $\left(\mathrm{X}_{1} \mathrm{X}_{2} \mathrm{Y}\right)$, metaphase I, the largest bivalent and one medium-sized bivalent bear a terminal NOR. The sex chromosome trivalent (separated by line) also includes NOR signal. Position of NOR is, however, unclear. Inset: diplotene, sex chromosome trivalent and its scheme, note NOR at the end of $X_{1}$ chromosome; $\mathbf{c}$, $\mathbf{d}$ Belisana sabah (X0), $\mathbf{c}$ Metaphase I. Five bivalents contain NOR, sex chromosome with NOR at both ends. d Incomplete mitotic metaphase (21 chromosomes), NORs terminal, $X$ chromosome with NOR at both ends; $\mathbf{e}, \mathbf{f}$ Pholcus phalangioides $\left(\mathrm{X}_{1} \mathrm{X}_{2} \mathrm{Y}\right.$ ). e Metaphase I, three bivalents contain NOR. The sex chromosome trivalent contains the signal in region of chromosome pairing (see scheme). $\mathbf{f}$ Mitotic metaphase, NORs terminal. $X_{1}$ chromosome bears NOR at both ends. Number of the other NOR-bearing chromosomes (seven) suggests that the $X_{2}$ chromosome contains a NOR too. Inset: another mitotic metaphase, $Y$ chromosome considerably condensed, without NOR. Bar $=5 \mu m$; insets $1 \mu \mathrm{m}$

file 22: Fig. S17f) and S. cylindrogaster $\left(2 \mathrm{n}^{\star}=26, \mathrm{X}_{1} \mathrm{X}_{2} 0\right)$ (Fig. 11e). The metacentric $X_{1}$ chromosome was the largest element of the karyotype, except for three Smeringopus species. The $\mathrm{X}_{2}$ chromosome was usually a medium-sized element (Additional file 1: Table S1). It had a variable morphology, being metacentric in $S$. ndumo, S. pallidus and S. similis, submetacentric in $S$. atomarius, subtelocentric in Hoplopholcus and S. peregrinus, and acrocentric in S. cylindrogaster and Smeringopus sp. (Additional file 1: Table S1). The metacentric $\mathrm{X}_{3}$ chromosome of $S$. pallidus was the smallest chromosome of the karyotype (Additonal file 1: Table S1).

Representatives of the genera Crossopriza, Holocnemus, and Stygopholcus exhibited the X0 system, including a metacentric X chromosome (Additional file 19:
Table S5, Fig. 11a-c, g, Additional file 19: Fig. S16, Additional file 23: Fig. S18), which was the largest element of the set except for Crossopriza sp. and Holocnemus caudatus (Additional file 1: Table S1).

Smeringopines exhibited a considerable diversity of the NOR number (Additional file 19: Table S5). Their karyotypes contained one (H. hispanicus, Hoplopholcus species, S. skotophilus) (Fig. 12a, c, d, i), two (Holocnemus pluchei, S. atomarius, S. pallidus) (Figs. 8c, 12b, e, f) or four (Smeringopus sp.) (Fig. 12g, h) chromosome pairs bearing a NOR. In most species, the morphology of these pairs and the NOR location was also determined. Chromosome pairs bearing NORs were biarmed; NORs were terminal (Additional file 19: Table S5). Smeringopus sp. exhibited an odd number of chromosomes bearing a 

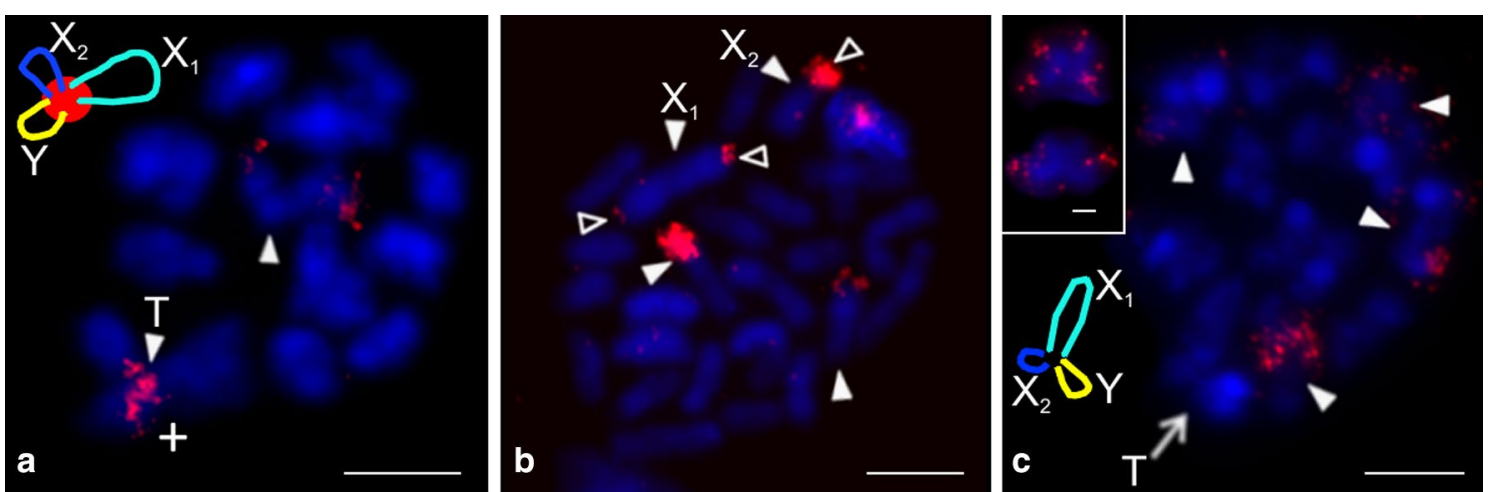

Fig. 10 Pholcinae, males, NORs (FISH) II. Figures $\mathbf{a}$, c contain scheme of sex chromosome trivalent $X_{1} X_{2} Y$ (red circle = signal formed by several NORs). Arrowhead $=$ NOR bearing chromosome $(\mathbf{b})$, bivalent $(\mathbf{a}, \mathbf{c})$ or trivalent $(\mathbf{a})$, open arrowhead = sex chromosome-linked NOR, $T=$ sex chromosome trivalent $X_{1}=X_{1}$ chromosome, $X_{2}=X_{2}$ chromosome, $Y=Y$ chromosome, $+=$ signal formed by several NORs. $\mathbf{a}, \mathbf{b}$ Muruta tambunan $\left(X_{1} X_{2} Y\right)$. a Metaphase I, note a bivalent bearing a terminal NOR and sex chromosome trivalent with signal in region of chromosome pairing (see scheme). b Mitotic metaphase, the large $X_{1}$ chromosome bears two terminal NORs, the $X_{2}$ chromosome contains one terminal NOR. Chromosomes of a chromosome pair exhibit another terminal NOR; c Pholcus pagbilao $\left(X_{1} X_{2} Y\right)$, metaphase I. Plate contains four NOR bearing bivalents and sex chromosome trivalent without signal (see scheme). Inset: metaphase II, NOR bearing pair, upper chromosome contains two terminal loci. Bar $=5 \mu \mathrm{m}$; inset $1 \mu \mathrm{m}$

NOR, which indicates heterozygosity of one pair for the presence of a NOR (Fig. 12h). The karyotype of H. hispanicus (X0) also contained a sex chromosome-linked NOR, which was placed at the end of the $\mathrm{X}$ chromosome (Fig. 12a).

\section{Chromosome behaviour in the male germline}

Males exhibited standard meiosis except for prophase I. Following pachytene, bivalents appeared globular and almost decondensed (so-called diffuse stage). In contrast, the sex chromosomes formed a highly spiralised and positively heteropycnotic body (i.e. stained more intensively than the other chromosomes) (Fig. 13a). In some pholcids, bivalents became considerably condensed during the late diffuse stage, whereas they retained a globular shape (e.g., Smeringopus). Despite condensation of bivalents, chiasmata were not discernible or only partially expressed (Fig. 13b). Following the diffuse stage, bivalents of some species uncoiled and exhibited a morphology typical for late prophase I (i.e., diplotene and diakinesis). Alternatively, the standard morphology of the bivalents emerged gradually during their condensation (Additional file 11: Fig. S8g). In some species, diplotene was reduced or even missing. In that case, bivalents showed a diakinetic morphology after the diffuse stage (Figs. 13c, 14b, c). In some pholcids, the bivalents (Metagonia, Additional file 11: Fig. S8e, f) or their middle part (Artema nephilit, Fig. 13c) retained a low condensation during late prophase I.

Male meiosis of most pholcids was remarkable for a very low chiasma frequency. The absolute majority of bivalents contained only a single chiasma (Additional file 2: Table S2, Additional file 7: Table S3, Additional file 12: Table S4, Additional file 19: Table S5, Fig. 14b-i, Additional file 11: Fig. S8d-f, h). An increased frequency of chiasmata (chiasma frequency $\geq 1.1$ per bivalent) was found only in artemines with a low diploid number, in Modisimus cf. elongatus (Modisiminae), Pholcus sp., and Quamtana filmeri (Pholcinae) (Additional file 2: Table S2, Additional file 7: Table S3, Additional file 12: Table S4, Fig. 13d-f). In Physocyclus dugesi (Arteminae) and $M$. cf. elongatus (Modisiminae), we also found rare bivalents containing three chiasmata (Fig. 13d). In some species, centromeric regions formed a knob or flexure on the bivalents during late prophase I, which allowed us to determine the relative position of the chiasmata and centromere (Fig. 13d, e). Chiasmata of most analysed pholcids were predominantly distal and intercalar. In Modisimus and Physocyclus, pericentric chiasmata were frequent or even predominant (Fig. 13d, e). A large bivalent displayed positive heteropycnosis in late prophase I of Pehrforsskalia and Pholcophora (Fig. 14b, Additional file 11: Fig. S8g).

The sex chromosomes exhibited a specific behaviour in the male germline, which was often already initiated in the spermatogonia. During spermatogonial mitosis, the sex chromosomes differed often by condensation and pycnosis from the other chromosomes. In some species, they were more condensed and positively heteropycnotic. This behaviour was frequent in modisimines and pholcines (Additional file 24: Fig. S19a, b). In some Pholcus species, the Y chromosome exhibited a more intensive 


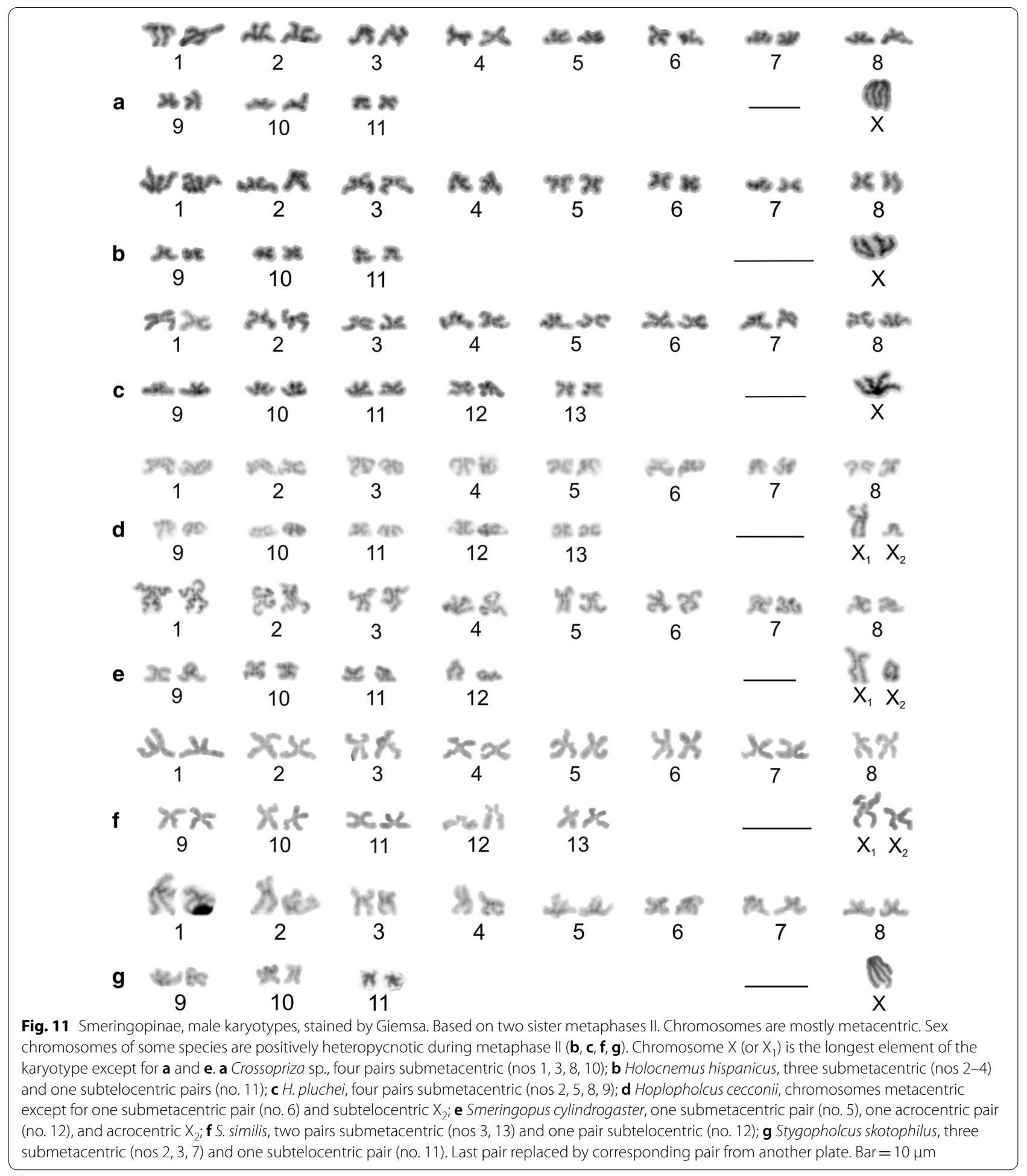

heteropycnosis than the $\mathrm{X}$ chromosomes. This $\mathrm{Y}$ chromosome pattern continued frequently at premeiotic interphase and meiosis (Fig. 14c-e, Additional file 11: Fig. S8i, Additional file 24: Fig. S19f, Additional file 25: Fig. S20a). In Artema, X chromosome condensation was delayed in comparison with the other chromosomes during spermatogonial metaphase (Additional file 24: Fig. S19c).

The sex chromosomes of some pholcids exhibited a specific location in the mitotic plate. In species with the 

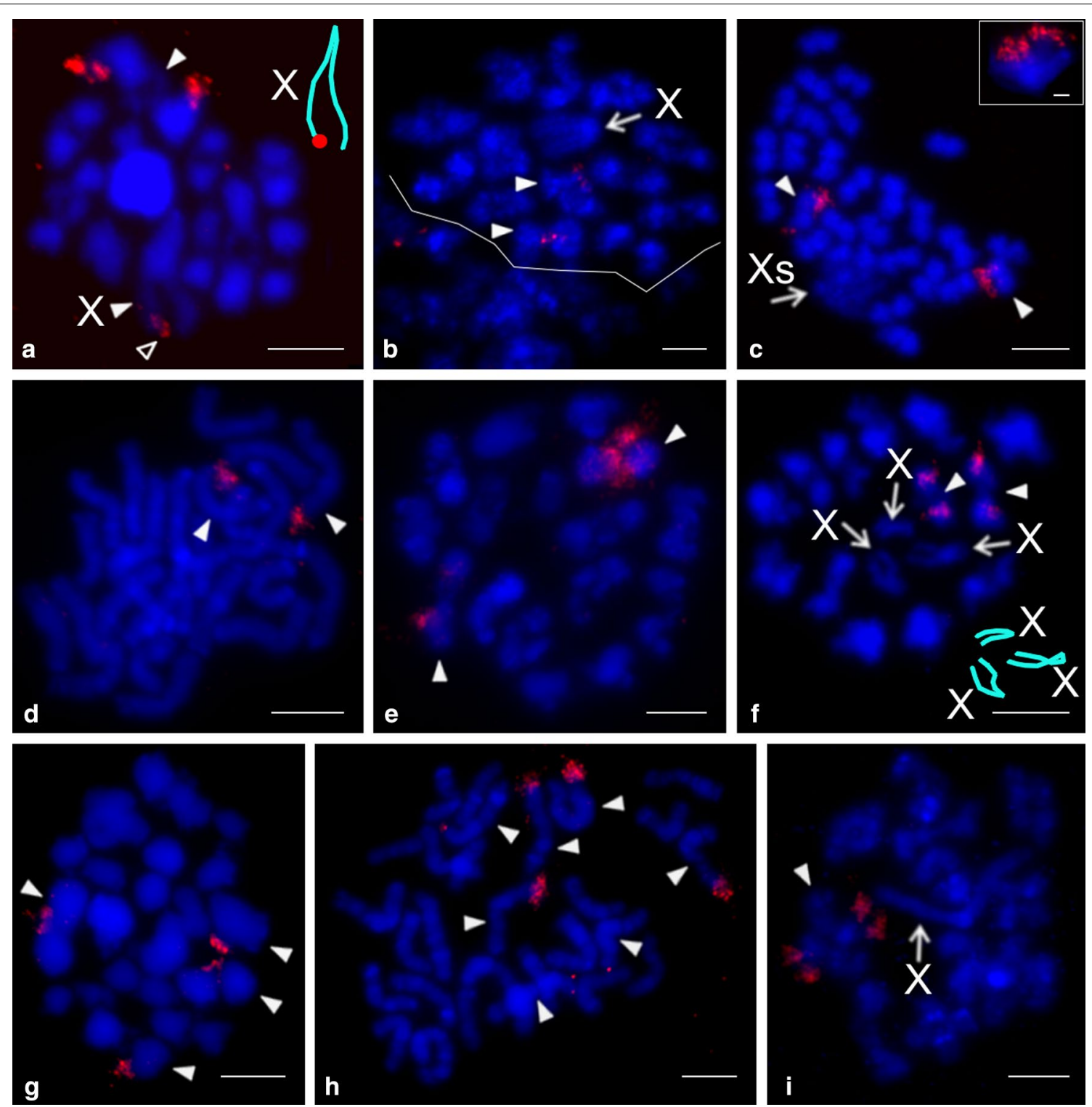

Fig. 12 Smeringopinae, males, detection of NORs (FISH). Arrowhead =NOR-bearing chromosome $(\mathbf{a}, \mathbf{c}, \mathbf{d}, \mathbf{h})$ or bivalent $(\mathbf{a}, \mathbf{b}, \mathbf{e}-\mathbf{g}, \mathbf{i})$, open arrowhead $=$ sex chromosome-linked NOR, $X=X$ chromosome, $X s=X$ chromosomes. a Holocnemus hispanicus (X0), metaphase I, the largest bivalent and one end of the $X$ chromosome bear a terminal NOR (see scheme of $X$ chromosome, red=NOR); $\mathbf{b}$ H. pluchei $(X 0)$, metaphase I (separated by a line from a diffuse stage), two bivalents containing a terminal chiasma have a NOR in chiasma region; $\mathbf{c}$ Hoplopholcus forskali $\left(X_{1} X_{2} 0\right)$, plate formed by two fused sister prometaphases II. Sex chromosomes form a cluster having a very low condensation. Chromosomes of one pair bear NOR. Inset: NOR-bearing chromosome from another metaphase II. This chromosome exhibits metacentric morphology and terminal location of NOR; $\mathbf{d}$ H. labyrinthi $\left(\mathrm{X}_{1} \mathrm{X}_{2} \mathrm{O}\right)$, mitotic metaphase. Chromosomes of one pair bear terminal NOR; e Smeringopus atomarius $\left(\mathrm{X}_{1} \mathrm{X}_{2} \mathrm{O}\right)$, metaphase I, note two bivalents with NOR; $\mathbf{f} S$. pallidus $\left(X_{1} X_{2} X_{3} 0\right)$, two bivalents with NOR. Note scheme of sex chromosome association; $\mathbf{g}$, $\mathbf{h}$ Smeringopus sp. $\left(X_{1} X_{2} 0\right)$, g metaphase I, four bivalents include NOR. $\mathbf{h}$ Mitotic metaphase, seven chromosomes show a terminal NOR; i Stygopholcus skotophilus (X0), metaphase I, one large bivalent bears a terminal NOR. Bar $=5 \mu \mathrm{m}$, inset $1 \mu \mathrm{m}$

$\mathrm{X}_{1} \mathrm{X}_{2} \mathrm{Y}$ system, they were often associated in the middle of the metaphase plate (Artema, Nipisa, Leptopholcus, Pholcus). While chromosomes $\mathrm{X}_{1}$ and $\mathrm{X}_{2}$ were often aligned in parallel (Additional file 24: Fig. S19b, c), chromosome $\mathrm{Y}$ was sometimes released from the association
(Additional file 24: Fig. S19b). Sex chromosomes were usually not discernible at spermatogonial mitoses of pholcids with the $\mathrm{X}_{1} \mathrm{X}_{2} 0$ system, due to the absence of heteropycnosis. In some species, however, it was possible to detect the prominent $\mathrm{X}_{1}$ chromosome. It was usually 
a
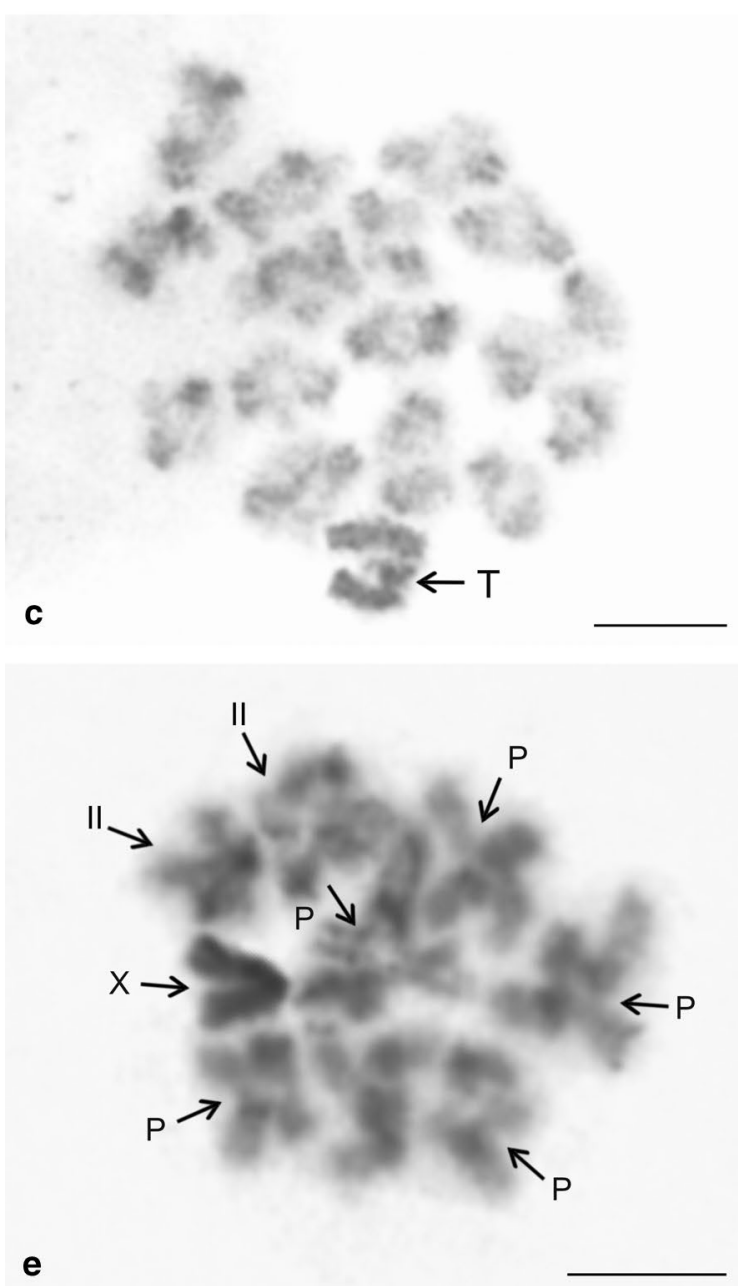

b

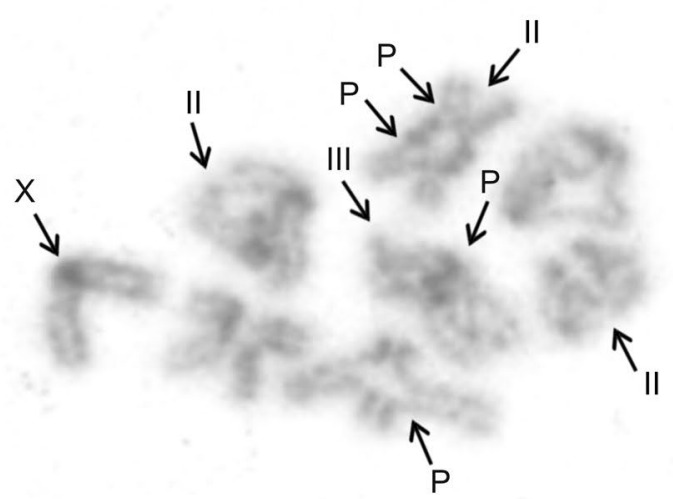

d

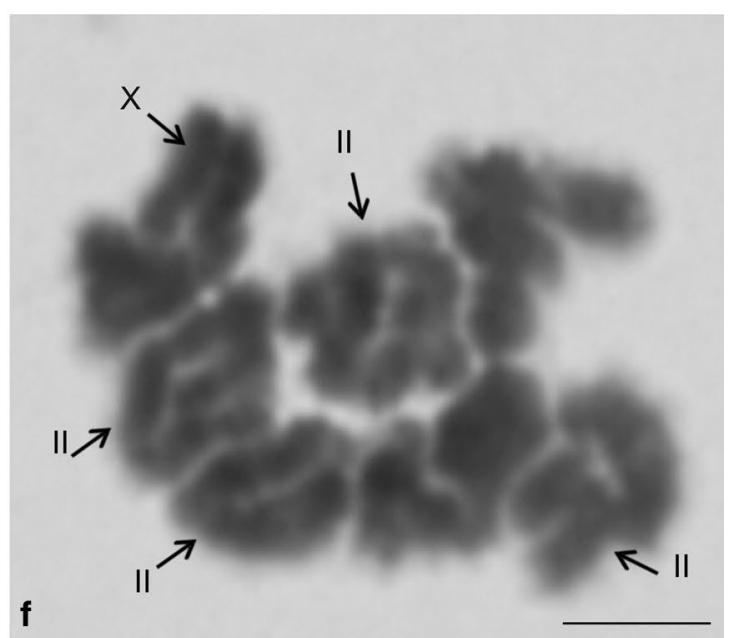

Fig. 13 Meiosis of pholcid males, diffuse stage and pattern of chiasmata. $P=$ pericentric chiasma, $T=$ sex chromosome trivalent, $X=X$ chromosome, $I I=$ bivalent with two chiasmata, $I I=$ bivalent with three chiasmata. a Artema nephilit $\left(X_{1} X_{2} Y\right)$, early diffuse stage. Bivalents considerably decondensed, sex chromosomes form positively heteropycnotic body; b Metagonia sp. $\left(X_{1} X_{2} Y\right.$ ), late diffuse stage, bivalents condensed but without well differentiated chiasmata. Sex chromosomes form a highly condensed body; $\mathbf{c}$ Artema nephilit $\left(\mathrm{X}_{1} \mathrm{X}_{2} \mathrm{Y}\right)$, diakinesis. Bivalents retain low condensation, except for terminal regions; $\mathbf{d}$ Physocyclus dugesi $(\mathrm{X} 0)$, diplotene. Some bivalents have a pericentric chiasma. Centromere regions are dark, more condensed; e Modisimus cf. elongatus (X0), diplotene. Sex chromosome is positively heteropycnotic. Most bivalents have pericentric chiasma. Centromeric regions are dark, more condensed; $\mathbf{f}$ Chisosa diluta (X0), metaphase I. Most bivalents have two chiasmata. Bar $=10 \mu \mathrm{m}$ 


\begin{abstract}
(See figure on next page.)
Fig. 14 Meiosis of pholcid males, sex chromosome pairing. Figures $\mathbf{b}-\mathbf{e}$ contain scheme of sex chromosome trivalent $X_{1} X_{2} Y$. $H=$ positively heteropycnotic bivalent, $X=X$ chromosome, $X_{1}=X_{1}$ chromosome, $X_{2}=X_{2}$ chromosome, $Y=Y$ chromosome. $\mathbf{a}$, $\mathbf{b}$ Pholcophora americana $\left(X_{1} X_{2} Y\right)$. $X$ chromosomes associated by ends of both arms with tiny $Y$ chromosome. a Premeiotic interphase. $\mathbf{b}$ Diakinesis. Note positive heteropycnosis of a large bivalent; $\mathbf{c}$ Pholcus kindia $\left(\mathrm{X}_{1} \mathrm{X}_{2} \mathrm{Y}\right)$, diakinesis. Sex chromosomes pair by ends of both arms. Condensation of the $\mathrm{X}$ chromosomes is much lower than that of the $Y$ chromosome; $\mathbf{d}$, e P. opilionoides $\left(X_{1} X_{2} Y\right)$, late prophase $\mathrm{I}, \mathrm{Y}$ chromosome shows a considerable condensation. $\mathbf{d}$ Early diplotene. Both ends of $X$ chromosomes involved in pairing. $\mathbf{e}$ Late diplotene. Both ends of $X_{1}$ and $Y$ chromosome take part in pairing. By contrast, only long arm of the $X_{2}$ chromosome is involved in pairing (c-centromeric knob of $X_{2}$ chromosome); $\mathbf{f}$ Hoplopholcus forskali $\left(X_{1} X_{2} 0\right)$, metaphase I, $X$ chromosomes pair by ends of both arms in the middle of the plate; $\mathbf{g}$ Cantikus sabah (X0), diplotene. Note parallel association of the $\mathrm{X}$ chromosome arms; $\mathbf{h}, \mathbf{i}$ Kambiwa neotropica $\left(\mathrm{X}_{1} \mathrm{X}_{2} \mathrm{X}_{3} \mathrm{X}_{4} \mathrm{Y}\right)$, metaphase I formed by 12 bivalents and a sex chromosome multivalent (in the middle of the plate). $\mathbf{h}$ positively heteropycnotic, cross-shaped sex chromosome multivalent is composed of two thick (1) and two thin "arms" (2). i Multivalent disintegrated into two rod-shaped structures ( $r$ ). Each of them consists of one thick and one thin "arm" (see scheme). Bar $=10 \mu \mathrm{m}$
\end{abstract}

placed in the middle of the plate (Additional file 24: Fig. S19d). We did not find any preferential location of the sex chromosome in spermatogonia of pholcids with the X0 system.

Meiotic pairing of sex chromosomes was already established in the premeiotic interphase. It was initiated by a parallel attachment of the $\mathrm{X}$ chromosomes (Additional file 24: Fig. S19e). Following completion of the pairing, sex chromosomes formed a body on the periphery of the nucleus. They retained their peripheral position during early prophase I (leptotene-pachytene) and the diffuse stage. In species with the $\mathrm{X}_{1} \mathrm{X}_{2} \mathrm{Y}$ and $\mathrm{X} 0$ systems, this position was often retained during late prophase I (Figs. 13c-f, 14b, Additional file 11: Fig. S8d-h, Additional file 25: Fig. S20a). In contrast, in species with multiple X systems, as well as in Kambiwa (complex SCS), the sex chromosomes were preferentially located in the middle of the plate in late prophase and metaphase I (Fig. 14f, h, i).

Chromosomes of the $\mathrm{X}_{1} \mathrm{X}_{2} \mathrm{Y}, \mathrm{XY}$, and multiple $\mathrm{X}$ systems paired without chiasmata in male meiosis. Biarmed sex chromosomes paired by the ends of both arms (Fig. 14c, f, Additional file 11: Fig. S8d, Additional file 15: Fig. S11e, Additional file 22: Fig. S17a, Additional file 26: Fig. S21a); the arms of each chromosome were often associated (Figs. 6c, 12f, 13c, 14b, Additional file 11: Fig. S8d). This pattern was also exhibited by the tiny $\mathrm{Y}$ chromosome of Aetana and Spermophora (Additional file 11: Fig. S8d). It was impossible to determine the pairing mode of the $\mathrm{Y}$ chromosome in other pholcids with a tiny size of this element. In some smeringopines with the $\mathrm{X}_{1} \mathrm{X}_{2} 0$ system and subtelocentric $\mathrm{X}_{2}$ chromosome, only the long arm of this element was involved in pairing in some plates during late prophase and metaphase I. Similarly, in some pholcines with a $\mathrm{X}_{1} \mathrm{X}_{2} \mathrm{Y}$ system and nonmetacentric $\mathrm{X}_{2}$ (Quamtana, Pholcus phalangioides), only the long arm of the $\mathrm{X}_{2}$ took part in pairing during this period (Additional file 15: Fig. S11c, g). In these species, all plates exhibited the same pattern of sex chromosome pairing. In P. opilionoides, pairing of the short arm of the
$\mathrm{X}_{2}$ chromosome was completed during early diplotene. Only the long arm of this chromosome was involved in pairing during late diplotene (Fig. 14d, e). In species with the $\mathrm{X} 0$ system, the arms of the $\mathrm{X}$ chromosome were usually associated with each other during late prophase and metaphase I (Figs. 5b, 12a, b, 13e, 14g, Additional file 5: Fig. S3c, e, Additional file 17: S13c, Additional file 23: Fig. 18a, c, e).

Our data suggest a complex sex chromosome pairing in Kambiwa $\left(\mathrm{X}_{1} \mathrm{X}_{2} \mathrm{X}_{3} \mathrm{X}_{4} \mathrm{Y}\right)$. During late prophase and metaphase I, sex chromosomes formed a cross-shaped multivalent consisting of four "arms" (Fig. 14h, Additional file 10: Fig. S7b). Two "arms" were thick and two were thin, which was most obvious in diakinesis (Additional file 10: Fig. S7a). The tiny Y chromosome was not discernible. It was only detected at spermatogonial mitosis and metaphase II (Additional file 11: Fig. S8a, c). In some plates, the multivalent disintegrated into two parts associated with each other. Each part was formed by one thick and one thin "arm" connected at one end (Fig. 14i).

Segregation of the sex chromosomes was usually delayed in anaphase I (Additional file 25: Fig. S20b). In some species, the sex chromosomes lagged even during telophase I. In species with two or three X chromosomes, the $\mathrm{X}$ chromosomes were arranged in parallel on the periphery of the plate during anaphase I. In contrast to the other chromosomes, chromatids of each X chromosome were associated during this period. Moreover, the arms of each X chromosome were also aligned (Additional file 25: Fig. S20b). The single $X$ chromosome of the X0 system exhibited the same pattern of chromatid and arm association. The association of chromatids and arms of particular X chromosomes was completed during prophase or metaphase II (Additional file 25: Fig. S20d). Association of the $\mathrm{X}$ chromosomes continued until the end of meiosis (Additional file 15: Fig. S11d, Additional file 22: Fig. S17b). The sex chromosome of Cantikus (X0) was remarkable for a precocious centromere division at metaphase II (Additional file 25: Fig. S20f). In contrast, the $\mathrm{X}$ chromosomes of Aetana $\left(\mathrm{X}_{1} \mathrm{X}_{2} \mathrm{Y}\right)$ exhibited slightly 


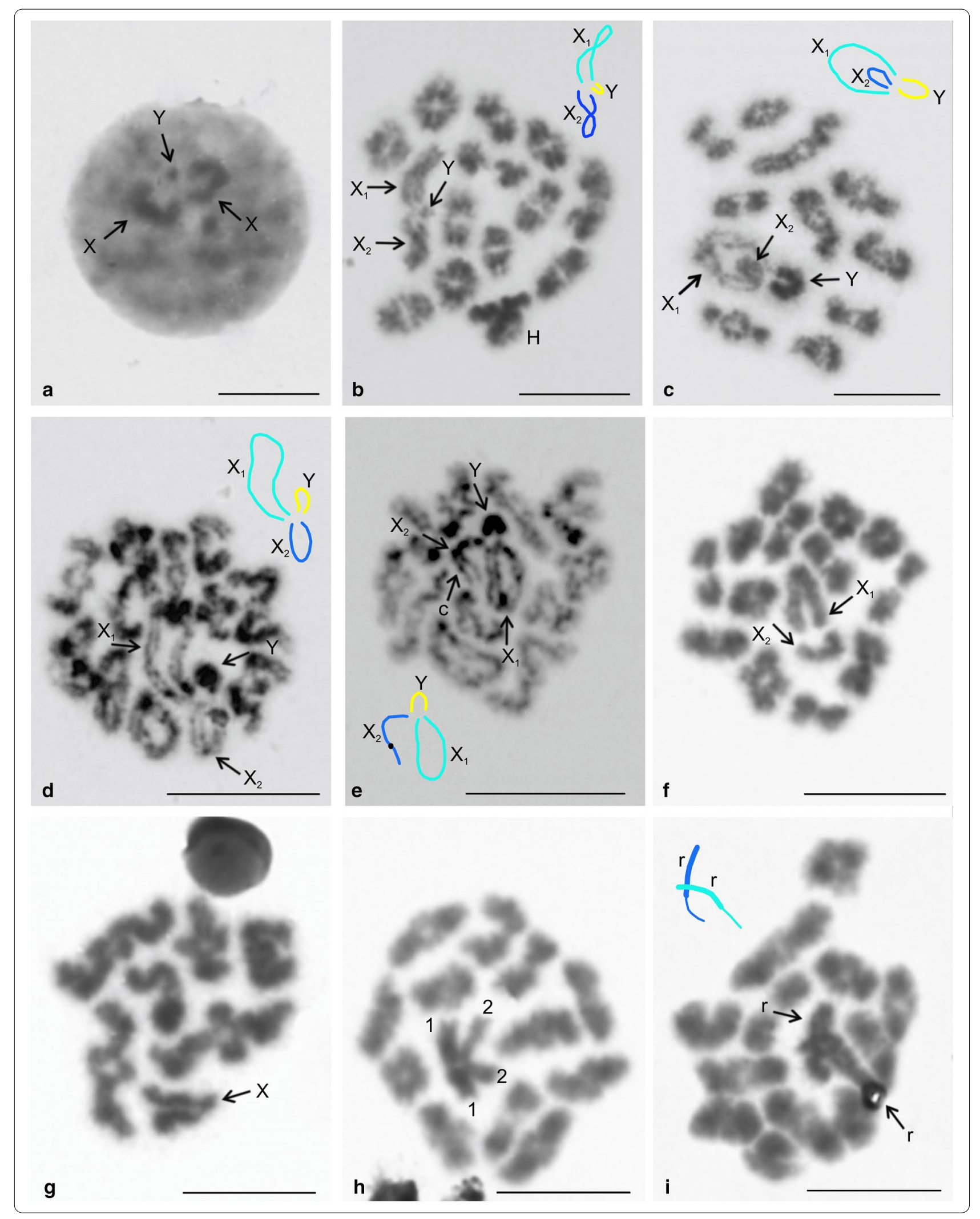


delayed centromeric division during this period (Additional file 25: Fig. S20g). In anaphase II, the segregation of the $\mathrm{X}$ chromosome(s) was slightly delayed in some pholcids, namely in Physocyclus (Arteminae), Psilochorus (Modisiminae), Holocnemus, and Hoplopholcus (Smeringopinae) (Additional file 25: Fig. S20i). Remarkably, the Y chromosome was placed preferentially in the middle of the plate from anaphase I until the end of meiosis in both $\mathrm{X}_{1} \mathrm{X}_{2} \mathrm{Y}$ and $\mathrm{XY}$ systems (Additional file 25: Fig. S20h, Additional file 26: Fig. S21b).

The sex chromosomes of each species showed a specific pattern of condensation and pycnosis during male meiosis. They became condensed and positively heteropycnotic during the premeiotic interphase (Fig. 14a, Additional file 24: Fig. S19e, f), forming a sex chromosome body (SCB). Sex chromosome condensation decreased considerably during the onset of prophase I, which was frequently accompanied by the disintegration of the SCB and loss of positive heteropycnosis. The following period of increase of condensation and pycnosis of the sex chromosomes culminated during the pachytene and diffuse stage, which was often accompanied by restoration of the SCB (Fig. 13a, b). Condensation of the sex chromosomes was often considerably reduced during the transition from the diffuse stage to late prophase I, which was accompanied by disintegration of the SCB. In some taxa, recondensation of the $\mathrm{X}$ chromosomes was delayed during late prophase I in comparison to the bivalents and Y chromosome. This was the case in Pholcophora (Ninetinae), Leptopholcus, Metagonia, Spermophora, and Pholcus (except for P. opilionoides and P. phalangioides) (Pholcinae) $\left(\mathrm{X}_{1} \mathrm{X}_{2} \mathrm{Y}\right)$, where the $\mathrm{X}$ chromosomes continued low condensation until diakinesis (Figs. 9a, 14c, Additional file 11: Fig. S8e, f, Additional file 25: Fig. S20a). On the other hand, the sex chromosome(s) of some pholcids (e.g. Holocneminus, Cantikus, Pehrforsskalia) displayed no or only a slight transient decrease of condensation during the transition from diffuse stage to diplotene (Fig. 14g, Additional file 11: Fig. S8g, Additional file 25: Fig. S20c). The sex chromosomes of Cantikus formed a highly condensed SCB until early metaphase I.

The sex chromosomes were considerably condensed and positively heteropycnotic during interkinesis (i.e. interphase between the first and second meiotic division). In Holocneminus, this pattern persisted until prometaphase II (Additional file 25: Fig. S20e). In most other species, the sex chromosome condensation decreased considerably during the onset of prophase II. This event was often accompanied by the loss of positive heteropycnosis. The sex chromosomes of some species (e.g. Hoplopholcus forskali) were almost decondensed during prophase II. They recondensed suddenly during late prophase or prometaphase II (Additional file 25: Fig. S20d).
The sex chromosomes of some pholcids also showed positive heteropycnosis during metaphase and anaphase II (e.g. Additional file 25: Fig. S20h, i).

Besides the germline cells, pholcid testes also frequently contained endopolyploid nuclei, whose size exceeded that of the diploid nuclei considerably. In some species, the sex chromosomes were positively heteropycnotic and formed a cluster in these nuclei (Additional file 27: Fig. S22).

\section{Discussion}

\section{Diploid numbers and morphology of chromosome pairs Evolution of chromosome numbers}

Male diploid numbers of pholcids range from 9 (three Micropholcus species) [25, this study] to 33 (Artema spp.) [this study]. The diploid number of the Micropholcus species is the lowest one found in araneomorph spiders with monocentric chromosomes so far [25]. Particular pholcid subfamilies differ by their range of numbers of chromosomal pairs (NCPs). Reported data suggest a low diversity of NCPs in modisimines (7-8 pairs) [25-27, this study], ninetines (12-13 pairs) [this study], and smeringopines (11-13 pairs) [9, 28-32, this study]. The other two subfamilies show a much higher range of NCPs: artemines from 6 to 15 pairs [30,32, 33, this study] and pholcines from 4 to 11 pairs [25, this study]. A high range of NCPs in artemines could reflect the possible non-monophyly of this group (see Fig. 15). In pholcines, there are also reports on 12 chromosome pairs in two species, but these data are dubious. The number of chromosome pairs in the species analysed by Sharma and Parida [34] can not be verified from the published information. In the species studied by Wang et al. [35], the presented data do not allow to determine unequivocally the NCPs and SCS.

Remarkably, the karyotype of an early-diverging pholcid, Artema (2n ${ }^{\star}=33, \mathrm{X}_{1} \mathrm{X}_{2} \mathrm{Y}$, chromosomes biarmed) is very similar to karyotypes of the haplogynes Filistata insidiatrix (Filistatidae; $2 \mathrm{n} \mathrm{O}^{-}=33, \mathrm{X}_{1} \mathrm{X}_{2} \mathrm{Y}$ ) [9], Paculla sp. (Pacullidae; 2n ${ }^{\star}=33, \mathrm{X}_{1} \mathrm{X}_{2} \mathrm{Y}$ ) [14], and Hypochilus pococki (Hypochilidae; 2n $\mathrm{O}^{-}=29, \mathrm{X}_{1} \mathrm{X}_{2} \mathrm{Y}$ ) [9]. Whereas Artema, Filistata, and Paculla exhibit the same diploid number and biarmed morphology of chromosome pairs, the karyotype of Hypochilus is slightly different, showing lower NCPs and a higher ratio of monoarmed chromosome pairs. The karyotype of Hypochilus can be derived from the pattern found in Artema, Filistata, and Paculla by two chromosome fusions and several pericentric inversions. This pattern of karyotypes suggests that the ancestral pholcid karyotype was close to those found in Artema, Filistata and Paculla $\left(2 \mathrm{n}^{*}=33, \mathrm{X}_{1} \mathrm{X}_{2} \mathrm{Y}\right.$, chromosomes biarmed).

Recent studies on spider phylogenomics suggest two primary lineages of haplogynes, one consisting of 


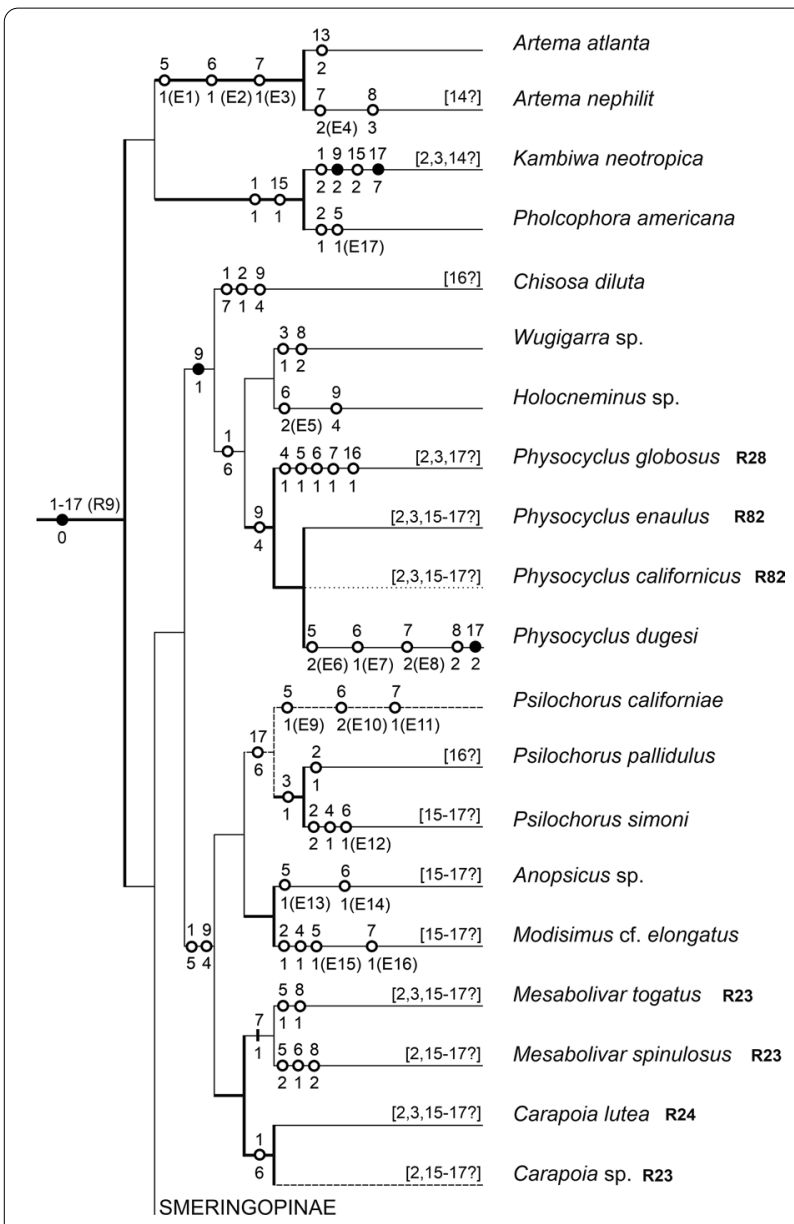

Fig. 15 Arteminae, Modisiminae, and Ninetinae: karyotype characters mapped on a cladogram. Based on the most recent molecular phylogeny of Pholcidae [20, 21]. Standard continuous lines: low bootstrapping branch support, i.e. < 70\%; thick continuous lines: modest to full support of hypotheses, $\geq 70 \%$ [21]; discontinuous lines: taxa not included in molecular phylogeny - phylogenetic position is based on cytogenetic data (dashed lines) or unresolved (dotted lines). Number above mark: number of character; number below mark: character state. Full marks: characters without homoplasy; empty marks: homoplasic characters. In some species, data on some characters are missing (information in square brackets). Selected events are marked $\mathrm{E}$ and numbered (see Additional file 1:Table S1 for chromosomes formed by these events). Chromosomes formed by the other events are identifiable unequivocally in Additional file 1: Table S1. Concerning chromosome pairs, only well-defined events changing chromosome morphology substantially are considered. Reconstruction of karyotype evolution is based on our data, except for taxa marked by the formula RX (see reference $X$ for information). See "Methods" (pp. 32-33) for coding of characters

filistatids and hypochilids, and another one comprising synspermiate haplogynes, including pholcids and pacullids [10, 36, 37] (Fig. 1). Considering the placement of Artema, Filistata, Paculla, and Hypochilus in this phylogeny, the karyotype structure found in the three first genera ( $2 \mathrm{n} \mathrm{o}^{\hat{*}}=33, \mathrm{X}_{1} \mathrm{X}_{2} \mathrm{Y}$, biarmed chromosomes) could be also the ancestral haplogyne karyotype. However, this conclusion conflicts with the hypothesis supposing that the ancestral karyotype of haplogynes was very close to that of entelegynes (20 chromosome pairs $\left.+X_{1} X_{2} 0\right)$ [9]. This hypothesis is supported by the occurrence of very similar karyotypes in the haplogyne family Drymusidae (17 biarmed pairs $+\mathrm{X}_{1} \mathrm{X}_{2} \mathrm{Y}$ ) and the family Austrochilidae (18 biarmed pairs $+\mathrm{XY}$ ) [9], which is an early-diverging lineage of a clade formed by protoentelegynes and entelegynes [37]. The observed karyotype pattern of austrochilids and drymusids could reflect an early separation of drymusids within haplogynes, specifically before derivation of the clade including filistatids, hypochilids, pacullids, and pholcids. To determine the correct phylogenetic position of drymusids, more detailed phylogenomic analysis of early-diverging araneomorph clades is needed.

A fundamental trend of spider karyotype evolution is the reduction of chromosome numbers, which took place independently in many clades [38]. Our analysis suggests the same pattern in pholcids. Available data indicate the reduction to 13 chromosome pairs in the last common ancestor of smeringopines and pholcines, and separately in ancestral ninetines, or alternatively in the last common ancestor of ninetines, smeringopines and pholcines (see "Phylogenetic implications", p. 29). Furthermore, a comparison of cytogenetic and molecular data suggests a further reduction to 11 pairs in ancestral pholcines, to 8 pairs in the common ancestor of modisimines, and to 7 pairs in the common ancestor of the artemine genera Holocneminus, Physocyclus, and Wugigarra (Fig. 15). The longest one or two chromosome pairs of pholcids are often prominent, which could reflect the formation of these pairs by fusion. In Leptopholcus $\left(2 \mathrm{n}^{-}=17\right.$, $\mathrm{X}_{1} \mathrm{X}_{2} \mathrm{Y}$ ), the first three pairs are considerably longer than the remaining ones. This set could be derived by three fusions from a karyotype containing ten chromosome pairs. Reductions of chromosome numbers also took place during the evolution of pholcid genera. However, the frequency of these events on genus level is low (Additional file 1: Table S1).

Variation of NCPs has also been suggested on intraspecific level in pholcids, namely in Crossopriza lyoni [30]. This widespread synanthropic species has been karyotyped by several authors. Recent studies consistently report 11 biarmed chromosome pairs in this species; analysed populations came from Brazil, India, and Vietnam [30, 32-34, 39, this study]. This number of pairs is probably an ancestral feature of the clade formed by Crossopriza, Holocnemus caudatus, H. hispanicus, and Stygopholcus (Fig. 16). In contrast, initial cytogenetic studies on C. lyoni suggested a higher NCPs in Indian populations, namely 12 [29] or 13 [28]. While 


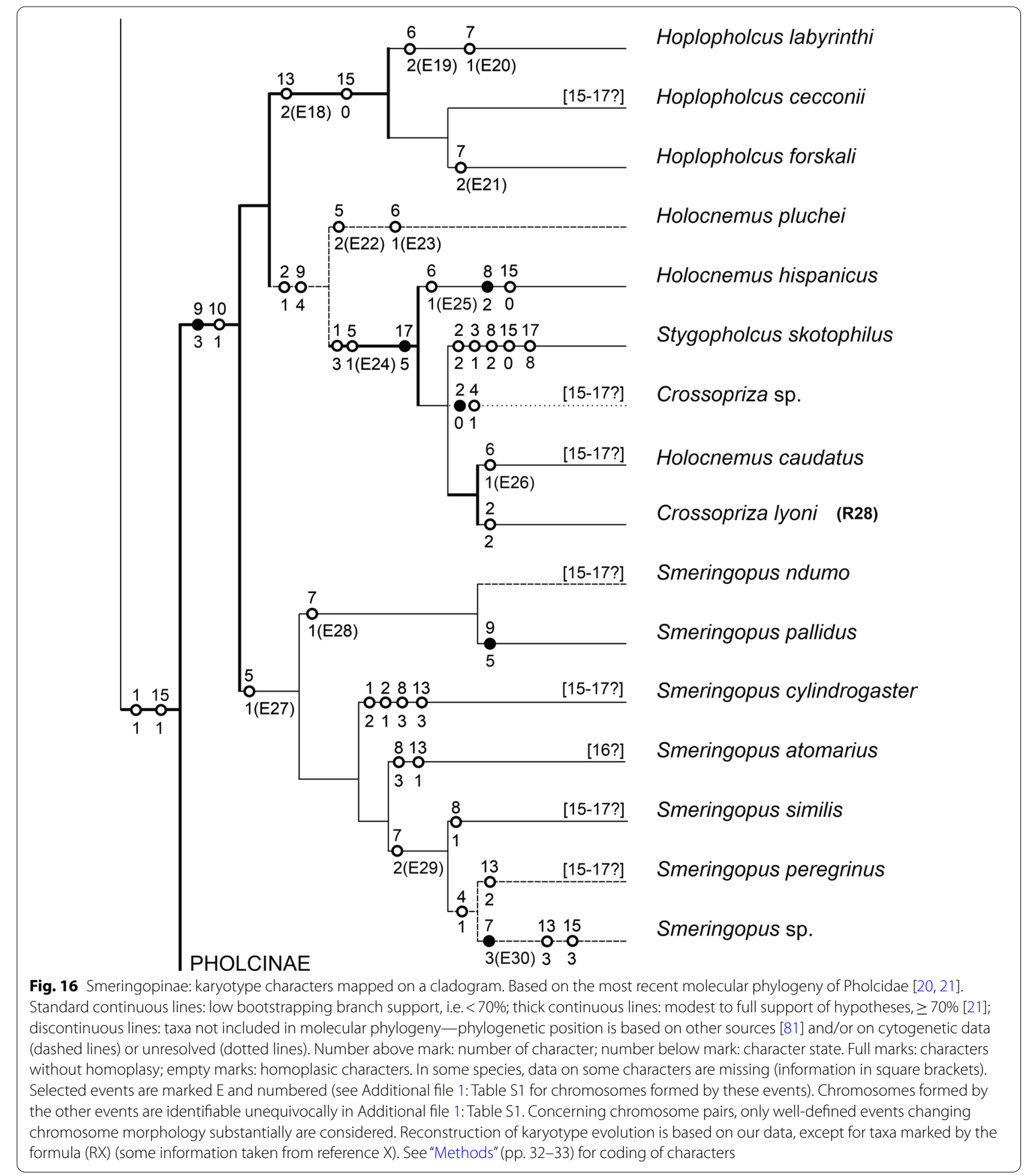

the first study does not contain information on chromosome morphology, the karyotype with 13 pairs was exclusively biarmed. This pattern could be derived from a set with 11 biarmed pairs by fissions of two pairs and subsequent inversions of four newly formed pairs. However, our results indicate a very low frequency of chromosome fissions during pholcid evolution and a low intraspecific variability of pholcid karyotypes. Therefore, 
differentiation of the karyotype of $C$. lyoni by multiple fissions is very unlikely. Diversity of NCPs in C. lyoni is most probably an artifact, possibly caused by species misidentifications. Reported data on NCPs also differ in Micropholcus fauroti. While Araujo et al. [26] reported eight metacentric pairs in a Brazilian population, we found only four metacentric pairs in another Brazilian population and African populations (Cape Verde, South Africa). Notably, we frequently found in our preparations of Micropholcus clusters formed by the fusion of chromosome plates. This artefact, along with the low number of observed plates, could have led Araujo et al. [26] to report double NCPs in this species. Four chromosome pairs were also reported in other karyotyped Micropholcus species [25].

\section{Evolution of chromosome pairs}

Similarly to the majority of other haplogynes with monocentric chromosomes [9], karyotypes of most pholcids are predominated by metacentric chromosomes. A different pattern has been reported only in two Physocyclus species [30, this study] and Pholcus manueli [35]. The karyotype of the latter species is suggested to be composed exclusively of monoarmed chromosomes. However, this idea is based only on the pattern of constitutive heterochromatin, i.e. a marker that could also be localised in regions other than the centromere. Therefore, this hypothesis should be tested by determination of the centromere position during mitotic metaphase or metaphase II. During metaphase II, chromatids of spider chromosomes are separated, except for the centromere.

At first glance, chromosome pairs of pholcids seem to be conservative elements, due to their predominantly metacentric morphology. However, comparison of closely related species revealed a dynamic nature of these pairs. Closely related taxa having the same number of pairs often differ by the morphology of one or several pairs, whose metacentric morphology is changed to submetacentric or even monoarmed (Additional file 1: Table S1). This pattern suggests the operation of pericentric inversions or some translocation variants. In some cases, the size of the pair is retained even after the change of its morphology to non-metacentric (e.g., acrocentric pair of Artema nephilit), which points to the operation of pericentric inversions. An enormous increase of the first pair (Crossopriza lyoni, Holocnemus caudatus) or considerable reduction of the last pair (some Psilochorus species, Stygopholcus, Wugigarra [this study], Mesabolivar spinulosus [25]), which are not accompanied by changes of NCPs, probably reflect an origin of these pairs by nonreciprocal or unequal reciprocal translocations. In the last three taxa, the last pair exhibits a subtelocentric morphology, which probably reflects translocation of most of an arm of an original biarmed pair to another chromosome. In Aetana, a considerable reduction of the last pair is accompanied by a change in morphology to acrocentric. This pattern could reflect centric fission of a biarmed chromosome pair, followed by integration of one product into another chromosome.

Diploid numbers of pholcids could be decreased by centric fusions of monoarmed pairs. Beside this, the number of pairs could be reduced by nested fusions. In contrast to centric fusions, this process involves biarmed chromosomes.

In conclusion, our data suggest the frequent involvement of fusions, inversions, and translocations of chromosome pairs in the karyotype evolution of pholcids. These kinds of rearrangements can be very effective in the formation of interspecific reproductive barriers [40, $41]$, and may thus have played a role in speciation processes in pholcids.

\section{Sex chromosomes $X_{1} X_{2} Y$ system}

Ancestral composition and origin of the $X_{1} X_{2} Y$ system Pholcids exhibit a considerable diversity of sex chromosomes. We found six SCS in these spiders, namely $\mathrm{X} 0, \mathrm{X}_{1} \mathrm{X}_{2} 0, \mathrm{X}_{1} \mathrm{X}_{2} \mathrm{X}_{3} \mathrm{O}, \mathrm{XY}, \mathrm{X}_{1} \mathrm{X}_{2} \mathrm{Y}$, and $\mathrm{X}_{1} \mathrm{X}_{2} \mathrm{X}_{3} \mathrm{X}_{4} \mathrm{Y}$. The $\mathrm{X}_{1} \mathrm{X}_{2} \mathrm{Y}$ system has been reported in seven haplogyne families, namely Sicariidae $[9,42,43]$, Filistatidae $[9,14-16]$, Drymusidae, Hypochilidae, Pholcidae [9], Pacullidae [14], and Plectreuridae [15]. Specific morphology and the meiotic pairing of chromosomes $\mathrm{X}_{1}, \mathrm{X}_{2}$, and $\mathrm{Y}$ suggest that the $\mathrm{X}_{1} \mathrm{X}_{2} \mathrm{Y}$ system arose once in haplogynes [9]. Together with molecular and paleontological data, this points to an ancient origin of the haplogyne $\mathrm{X}_{1} \mathrm{X}_{2} \mathrm{Y}$ system. Plectreurids have been found in Jurassic strata [44]. A recent molecular phylogeny suggests the origin of spiders possessing an $\mathrm{X}_{1} \mathrm{X}_{2} \mathrm{Y}$ system during the early Mesozoic [37]. The phylogenetic distribution of the $\mathrm{X}_{1} \mathrm{X}_{2} \mathrm{Y}$ system using recent phylogenomic trees $[10,36,37]$ suggests that this sex chromosome determination is ancestral for haplogynes, including pholcids. It could have arisen even earlier, namely before the separation of entelegyne and haplogyne spiders [16] or before the separation of mygalomorphs and araneomorphs in ancient opisthothele spiders. If so, the supposed ancestral sex chromosome system $\mathrm{X}_{1} \mathrm{X}_{2} 0$ of mygalomorphs [6] and entelegynes [9] arose from the $\mathrm{X}_{1} \mathrm{X}_{2} \mathrm{Y}$ system by the loss of the $\mathrm{Y}$ chromosome. The ancestral $\mathrm{X}_{1} \mathrm{X}_{2} \mathrm{Y}$ system probably consisted of two large metacentric $\mathrm{X}$ chromosomes of similar size and a metacentric $\mathrm{Y}$ microchromosome [9]. Species with this pattern have been found in almost all $\mathrm{X}_{1} \mathrm{X}_{2} \mathrm{Y}$ families $[9,16$, 18 , this study]. The $X_{1} X_{2} Y$ system was supposed to arise by rearrangements between autosomes and sex chromosomes [43]. In this case, it would be an ancient neo-sex 
chromosome system. According to our hypothesis, chromosomes $\mathrm{X}_{1}, \mathrm{X}_{2}$, and $\mathrm{Y}$ are derived from CSCP. It was suggested that multiple $\mathrm{X}$ chromosomes of spiders arose by nondisjunctions of the $\mathrm{X}$ chromosome of the CSCP [7]. The $Y$ chromosome could originate in a similar way, namely by a nondisjunction of the $\mathrm{Y}$ chromosome of the CSCP and subsequent degeneration of the newly formed element. Nondisjunctions could be a major mechanism of formation of multiple $\mathrm{X}$ chromosomes in spiders [7, 18, 45]. This unusual origin of sex chromosomes is supported by the inactivation of multiple $\mathrm{X}$ chromosomes during meiosis of spider females. This unique behaviour probably evolved to avoid the negative effects of duplicated X chromosomes on female meiosis $[5,7]$.

According to our data, chromosomes of the $\mathrm{X}_{1} \mathrm{X}_{2} \mathrm{Y}$ system are dynamic elements. Although they exhibit a conservative pairing during male meiosis, they underwent frequent rearrangements during pholcid evolution. Remarkably, chromosomes $\mathrm{X}_{1}, \mathrm{X}_{2}$, and $\mathrm{Y}$ differ by their pattern of morphological evolution and evolutionary plasticity. See Additional file 28: Appendix S1 for evolution of particular sex chromosomes of the $\mathrm{X}_{1} \mathrm{X}_{2} \mathrm{Y}$ system.

Conversion of the $X_{1} X_{2} Y$ system into other sex chromosome systems In some haplogynes, including pholcids, the $\mathrm{X}_{1} \mathrm{X}_{2} \mathrm{Y}$ system has been transformed into other SCS $\left[9,15\right.$, this study]. According to [9], the $X_{1} X_{2} Y$ system underwent conversion to the $\mathrm{X} 0$ system, namely by $\mathrm{X}$ chromosome fusion (formation of the XY system) and subsequent loss of the $\mathrm{Y}$ chromosome. Our results suggest that patterns of conversion of the $\mathrm{X}_{1} \mathrm{X}_{2} \mathrm{Y}$ to the $\mathrm{X} 0$ system are more diversified. In some pholcids, loss of the $\mathrm{Y}$ chromosome preceded fusion of the $\mathrm{X}$ chromosomes, which resulted in the formation of the $\mathrm{X}_{1} \mathrm{X}_{2} 0$ and, subsequently, of the $\mathrm{X} 0$ system. We suppose that this scenario could be more frequent during the evolution of the haplogyne $\mathrm{X}_{1} \mathrm{X}_{2} \mathrm{Y}$ system, due to specific features of its $\mathrm{Y}$ chromosome, which could facilitate degeneration and extinction of this element (tiny size, absence of recombinations between the $\mathrm{X}$ and $\mathrm{Y}$ chromosome).

\section{$X Y$ system}

In Wugigarra the $\mathrm{X}_{1} \mathrm{X}_{2} \mathrm{Y}$ system was transformed into the XY system by $\mathrm{X}$ chromosome fusion [this study]. The $\mathrm{XY}$ system of another haplogyne, Diguetia (Diguetidae), is suggested to arise by the same process [9]. The sex chromosomes of Wugigarra and Diguetia retained their metacentric morphology, except for the acrocentric X chromosome of $D$. canities, which probably originated from metacentric $X$ by pericentric inversion [9]. The $Y$ chromosomes of these haplogynes are small elements. While sex chromosomes of Wugigarra retain their achiasmatic pairing by both ends, only one $\mathrm{X}$ chromosome end is involved in achiasmatic pairing in Diguetia [9]. Notably, the XY system has also been suggested in other pholcids, namely Smeringopus ndumo (reported as S. pallidus) [9] and Holocnemus pluchei [31]. Our data showed that these reports are erroneous, based on revision of original preparations (S. ndumo) and laboratory stock studied by previous authors (H. pluchei). An XY system was also discovered in several other spiders $[6,9,46,47]$. Its origin is, however, different. It is suggested to have arisen from the $\mathrm{X} 0$ determination by a fusion of the $\mathrm{X}$ chromosome and an autosome(s), resulting in the formation of neo-X and neo-Y chromosomes. Another possible origin of the system might be by a fusion of the $\mathrm{X}$ chromosome and X chromosome of the CSCP.

\section{Multiple $X$ chromosome systems}

$X_{1} X_{2} O$ system Although the $\mathrm{X}_{1} \mathrm{X}_{2} 0$ system is the most frequent sex chromosome determination in entelegyne araneomorphs $[18,45]$, it is rare in haplogyne araneomorphs $\left[9,15\right.$, this study]. Provided that the $\mathrm{X}_{1} \mathrm{X}_{2} \mathrm{Y}$ system is ancestral for araneomorphs [16], the $\mathrm{X}_{1} \mathrm{X}_{2} 0$ system has originated in the same way in both entelegynes and haplogynes, namely from the $\mathrm{X}_{1} \mathrm{X}_{2} \mathrm{Y}$ system by the loss of the $\mathrm{Y}$ chromosome. The $\mathrm{X}_{1} \mathrm{X}_{2} \mathrm{Y}$ system of the pholcids Aetana and Artema, including a minute $\mathrm{Y}$ chromosome [this study], could represent an evolutionary transition between the $\mathrm{X}_{1} \mathrm{X}_{2} \mathrm{Y}$ and $\mathrm{X}_{1} \mathrm{X}_{2} 0$ systems. In haplogynes, the $\mathrm{X}_{1} \mathrm{X}_{2} 0$ system has originated several times, namely in filistatids [15], plectreurids [9], and pholcids [this study].

In pholcids, we found the $\mathrm{X}_{1} \mathrm{X}_{2} 0$ system in two smeringopine genera, Hoplopholcus and Smeringopus. Mapping of the distribution of the $\mathrm{X}_{1} \mathrm{X}_{2} \mathrm{O}$ system in smeringopines suggests an origin of this system in their ancestor. Remarkably, we never found the $\mathrm{X}_{1} \mathrm{X}_{2} 0$ system in pholcids in which it was reported previously (see database of Araujo et al. [24]). Instead, these species exhibit the $\mathrm{X}_{1} \mathrm{X}_{2} \mathrm{Y}$ system, which had probably been mistaken for the $\mathrm{X}_{1} \mathrm{X}_{2} 0$ system, due to the small size of the $\mathrm{Y}$ chromosome. The $\mathrm{X}_{1} \mathrm{X}_{2} \mathrm{Y}$ system was revealed in pholcids less than two decades ago [9].

Remarkably, the $\mathrm{X}_{1}$ and $\mathrm{X}_{2}$ chromosomes of the $\mathrm{X}_{1} \mathrm{X}_{2} 0$ system show a similar evolution to the $\mathrm{X}$ chromosomes of the $\mathrm{X}_{1} \mathrm{X}_{2} \mathrm{Y}$ system in pholcids (see Additional file 28: Appendix S1 for evolution of chromosomes of the $\mathrm{X}_{1} \mathrm{X}_{2} \mathrm{Y}$ system). While the $X_{1}$ is a conservative element retaining large size and metacentric morphology, $\mathrm{X}_{2}$ underwent reduction and frequent changes of morphology. The size of the $\mathrm{X}_{1}$ chromosome is more variable $(7.9-15.1 \%$ of TCL) than that of the $\mathrm{X}_{2}$ element (5.1-7.4\% of TCL), which could reflect insertions of fragments derived from CPs into the $X_{1}$ chromosome. In each species, the $X_{2}$ chromosome is reduced in comparison with the $\mathrm{X}_{1}$ chromosome (including representatives with an ancestral 
state, i.e. metacentric morphology of the $\mathrm{X}_{2}$ chromosome). Therefore, the $\mathrm{X}_{2}$ chromosome was probably already reduced in an ancestor of smeringopines (having a $\mathrm{X}_{1} \mathrm{X}_{2} \mathrm{O}$ or $\mathrm{X}_{1} \mathrm{X}_{2} \mathrm{Y}$ system). The original metacentric $\mathrm{X}_{2}$ chromosome has been transformed several times to a monoarmed one, namely in the ancestor of Hoplopholcus and in several Smeringopus species. The morphology of the $\mathrm{X}_{2}$ chromosome has most probably changed by a pericentric inversion or translocation.

$X_{1} X_{2} X_{3} O$ system A sex chromosome system comprising three $\mathrm{X}$ chromosomes is relatively frequent in entelegyne [48] and mygalomorph spiders [6]. Among haplogynes, Smeringopus pallidus is the first reported species with this sex chromosome determination [this study]. It was obviously derived from the $\mathrm{X}_{1} \mathrm{X}_{2} 0$ system found in the other Smeringopus species. However, the origin of the third $\mathrm{X}$ chromosome of S. pallidus is unresolved. The behaviour of the three $\mathrm{X}$ chromosomes of this species during male meiosis (pairing, pattern of condensation and heteropycnosis) is the same as in $\mathrm{X}_{1} \mathrm{X}_{2} 0$ species, which contradicts the possible origin of the extra $\mathrm{X}$ chromosome from autosomes. Another possibility is nondisjunction of an $\mathrm{X}$ chromosome, which is supposed to be a mechanism of $\mathrm{X}_{1} \mathrm{X}_{2} \mathrm{X}_{3} \mathrm{O}$ formation in entelegynes [7, 45, 49]. This hypothesis is supported by the same meiotic behaviour of sex chromosomes in $\mathrm{X}_{1} \mathrm{X}_{2} \mathrm{O}$ and $\mathrm{X}_{1} \mathrm{X}_{2} \mathrm{X}_{3} 0$ species. The sex chromosomes of $S$. pallidus (two large metacentric $\mathrm{X}$ chromosomes of similar size + one small metacentric $\mathrm{X}$ chromosome) can be derived from the ancestral pattern of the $\mathrm{X}_{1} \mathrm{X}_{2} 0$ system in Smeringopus (two metacentric $\mathrm{X}$ chromosomes, $\mathrm{X}_{2}$ considerably smaller than $\mathrm{X}_{1}$ ) by nondisjunction of the $\mathrm{X}_{1}$ chromosome. Formation of an additional large $\mathrm{X}$ chromosome would substantially increase the sum of the relative lengths of the $\mathrm{X}$ chromosomes. However, the values do not differ substantially between S. pallidus (16.9\% of TCL) and the karyotypically most similar S. ndumo (18\% of TCL), and fall within the range of values found in other Smeringopus species (14.2$19.8 \%$ of TCL). Therefore, the $\mathrm{X}_{3}$ chromosome of S. pallidus more likely arose by a fission of the ancestral metacentric $\mathrm{X}_{1}$ chromosome into two acrocentric chromosomes, followed by their pericentric inversion. This hypothesis is supported (1) by a reduction of the $X_{1}$ chromosome of $S$. pallidus in comparison with other Smeringopus species, and (2) by the sum of $X_{1}$ and $X_{3}$ sizes in S. pallidus, which is similar to the $\mathrm{X}_{1}$ size in the karyotypically most similar $S$. ndumo. To resolve the origin of the $\mathrm{X}_{1} \mathrm{X}_{2} \mathrm{X}_{3} 0$ system in S. pallidus, sequencing of its $\mathrm{X}$ chromosomes needs to be carried out.

\section{XO system}

Phylogenetic distribution and origins of the pholcid XO system The X0 system is common in pholcids. It has been reported in some artemines, pholcines, smeringopines, and all modisimines karyotyped so far [24, this study]. Character mapping suggests at least five origins of the X0 system in pholcids, namely in the (1) artemines with low diploid numbers (see "Phylogenetic implications", p. 29), (2) ancestor of modisimines (Additional file 29: Fig. S23), (3) common ancestor of smeringopines Holocnemus, Crossopriza, and Stygopholcus (Additional file 30: Fig. S24), (4) pholcine Belisana, and (5) common ancestor of the pholcines Cantikus and Micropholcus (Additional file 31: Fig. S25). The X0 system was also reported in Pholcus manueli [35]. However, chromosome plates obtained in P. manueli (male mitoses) do not allow us to determine the SCS of this species unequivocally. Similar to $P$. phalangioides $\left(\mathrm{X}_{1} \mathrm{X}_{2} \mathrm{Y}\right)$ [9], the karyotype of $P$. manueli is composed of 25 chromosomes and contains an odd peculiar chromosome formed exclusively by constitutive heterochromatin [35]. The odd heterochromatic element of $P$. phalangioides is a Y chromosome [9]. Therefore, $P$. manueli exhibits with all probability the $\mathrm{X}_{1} \mathrm{X}_{2} \mathrm{Y}$ system.

The $\mathrm{X} 0$ system is common in spiders. Phylogenetic distribution of the $\mathrm{X} 0$ system in spiders suggests multiple origins of this system $[6,18,45]$. X0 systems of entelegynes and mygalomorphs arose by chromosome fusions from multiple $\mathrm{X}$ chromosome systems $[6,18,45]$. In haplogynes, the $\mathrm{X} 0$ system has been found in nine families (see database [24]). The X0 system of haplogynes with monocentric chromosomes arose from $\mathrm{XY}$ or $\mathrm{X}_{1} \mathrm{X}_{2} 0$ systems. The $\mathrm{X} 0$ system of smeringopine pholcids arose from the $\mathrm{X}_{1} \mathrm{X}_{2} 0$ system by $\mathrm{X}$ chromosome fusion [this study]. In contrast, the X0 system of a clade formed by Chisosa, Holocneminus, Physocyclus, and Wugigarra probably arose from the XY system (Additional file 29: Fig. S23). Formation of the X0 system in the other pholcids is unresolved. Our discovery of both X0 [this study] and ancestral $\mathrm{X}_{1} \mathrm{X}_{2} \mathrm{Y}$ systems [J. Král and $\mathrm{O}$. Košulič, unpublished] in the same genus, the pholcine Belisana, indicate a relatively fast formation of the $\mathrm{X} 0$ system during the evolution of this clade. An increase in the size of the $\mathrm{Y}$ chromosome during the evolution of pholcines (see Additional file 28: Appendix S1 for evolution of the Y chromosome) did not prevent this element from being subjected to reduction and loss in the common ancestor of Cantikus and Micropholcus (X0).

Evolution of the XO system in pholcids Despite its multiple origins, the single $\mathrm{X}$ chromosome of pholcids exhibits an extremely conservative morphology. Except for Modisimus it is always mediocentric (Additional file 1: Table S1), which indicates a strong selection pressure to keep this feature. We suppose that this morphology could be essential to ensure the self-association and regular segregation of the $\mathrm{X}$ chromosome univalent during male meiosis. In mice, the meiotic stability of the 
sex chromosome univalents is promoted by their selfassociation [50].

In contrast to morphology, the size of the $\mathrm{X}$ chromosome varies considerably, namely from 8.89 (Belisana sabah) to $21.21 \%$ of TCL (Chisosa diluta) (Additional file 1: Table S1). A considerable diversity of X chromosome sizes occurs even on a genus level (Crossopriza, Holocnemus, Psilochorus). The increase in size of the $\mathrm{X}$ chromosome could be a consequence of an integration of material from CPs, as suggested, for example, by the large difference of $\mathrm{X}$ chromosome size between two Holocnemus species (H. caudatus and H. hispanicus).

\section{$X_{1} X_{2} X_{3} X_{4} Y$ system}

Comparison of data on the male karyotype and meiosis suggests that the $\mathrm{X}_{1} \mathrm{X}_{2} \mathrm{X}_{3} \mathrm{X}_{4} \mathrm{Y}$ system occurs in Kambiwa. This pholcid exhibits one chromosome pair less than the other studied ninetine, Pholcophora, which displays a $\mathrm{X}_{1} \mathrm{X}_{2} \mathrm{Y}$ system [this study]. This pattern indicates the origin of the $\mathrm{X}_{1} \mathrm{X}_{2} \mathrm{X}_{3} \mathrm{X}_{4} \mathrm{Y}$ system by rearrangements between chromosomes of the $\mathrm{X}_{1} \mathrm{X}_{2} \mathrm{Y}$ system and a chromosome pair. The $\mathrm{Y}$ microchromosome was not involved in the rearrangements. Sex chromosome systems arising by rearrangements between chromosome pair(s) and sex chromosomes have also been reported in some other araneomorphs $[7,9,47,51-53]$ and in some mygalomorphs $[6,46]$. These events are always apomorphies of low-level taxa (species or species groups), which indicates their relatively recent origin.

\section{Sex chromosomes and pholcid speciation}

In summary, our data suggest frequent and diverse structural changes of sex chromosomes during pholcid evolution. These events include inversions and translocations of sex chromosomes, integration of fragments or even whole chromosomes into the SCS, and loss of the Y chromosome. Since structural changes of sex chromosomes are often very potent in formation of interspecific reproduction barriers [54-57], they have probably been involved in the speciation process in pholcids. In keeping with this view, closely related pholcids often differ in sex chromosome morphology [this study].

Particular sex chromosomes of pholcid males do not recombine, which suggests a high degree of differentiation of these chromosomes. This degree of sex chromosome differentiation is associated with markedly stronger reproductive isolation [58]. Although sex chromosome rearrangements could be less detrimental in meiosis of spider males due to achiasmatic sex chromosome pairing, in female heterozygotes they can lead to a collapse of homologous sex chromosome pairing.

\section{Nucleolus organizer regions NOR bearing pairs}

The pattern of NORs has only been determined in a low number of spiders so far. Usually, these structures were detected by silver staining, a technique that visualizes NORs that were active in the preceding interphase only [59]. Therefore, it is impossible to reconstruct the evolution of NORs in particular families or even higher taxa of spiders. Karyotypes of most spiders contain, however, a low number of these structures. The common ancestral pattern of opisthothele spiders comprised probably one or two chromosome pairs bearing a terminal NOR locus [6].

In our study, we analysed the evolution of spider NORs on a family level for the first time. Nucleolus organizer regions have been visualized only in three pholcids so far, in each case by silver staining [30, 32]. To reconstruct NOR evolution in pholcids, we analysed 30 pholcid species, including one species studied earlier by silver staining. To detect NORs, we used fluorescence in situ hybridization (FISH), which visualizes inactive NORs as well. According to our results, pholcids exhibit a considerable diversity of NOR numbers (from one to nine loci); congeneric species often differ in the number of NOR loci. Almost all detected NOR loci are homozygous for the presence of NOR, which suggests a high stability in the number of NOR loci at species level. Findings of loci heterozygous for the presence of NOR are rare in pholcids (Nipisa, Pholcus pagbilao, Smeringopus sp.) [this study]. In the case of Artema atlanta, intraspecific variability of NORs is doubtful. While the studied karyotype from South Africa contained one NOR locus [this study], two NOR loci were reported in an Indian population probed by silver staining [32]. Considering the low quality of the signals detected, the information of the latter authors should be verified by FISH.

Character mapping suggests that ancestral pholcids had a single biarmed CP bearing a terminal NOR (Additional file 29: Fig. S23). Whereas the number of NORs underwent multiple changes during pholcid evolution, the pattern of their location has remained conservative. Each NOR-bearing chromosome pair of pholcids includes a single NOR, except for exceptional pairs having NORs at both ends. These pairs were only found in some pholcines [this study]. NORs of pholcids are terminal, except for a pericentric NOR of Physocyclus globosus [30], which might originate from a paracentric inversion. The terminal location of pholcid NORs suggests that these structures have mostly spread by ectopic recombination. This mechanism could be promoted by hybridization of individuals belonging to populations differing in the number of NOR loci, which is indicated by 
the finding of heterozygotes for the presence of NOR in Nipisa, P. pagbilao, and, Smeringopus sp.

Our data indicate an increase in the number of NOR bearing CPs in some pholcid clades. In the ancestor of ninetines and the common ancestor of smeringopines and pholcines (Additional file 29: Fig. S23, Additional file 30: Fig. S24) or common ancestor of ninetines, smeringopines, and pholcines (see "Phylogenetic implications", p. 29), the number of NOR-bearing pairs increased to two. During subsequent evolution, NORs spread to three chromosome pairs in Kambiwa (Ninetinae) and in the ancestor of pholcines. Karyotypes of Smeringopus sp. and some pholcines contain four NOR-bearing pairs. In Belisana, there are even five pairs bearing NORs. On the other hand, character mapping suggests that the number of NOR-bearing pairs decreased several times in smeringopines (Stygopholcus, Holocnemus hispanicus, Hoplopholcus) and pholcines (Aetana, Muruta, Quamtana, common ancestor of Cantikus and Micropholcus) (Additional file 30: Fig. S24, Additional file 31: Fig. S25). Remarkably, these clades often exhibit reduced NCPs in comparison with their relatives; NORs retain a terminal position.

Sex chromosome-linked NORs In contrast to other opisthothele spiders, haplogynes often exhibit sex chromosome-linked NORs [9]. These NORs are also common in pholcids. They have been found in all pholcid subfamilies [30, this study]. Sex chromosome-linked NORs of pholcids and other haplogynes are always restricted to the X chromosome(s) [9, 30, this study] except for Nipisa [this study]. Character mapping suggests at least five origins of these NORs in pholcids, namely in (1) Physocyclus (Arteminae, X0), (2) Kambiwa (Ninetinae, $\mathrm{X}_{1} \mathrm{X}_{2} \mathrm{X}_{3} \mathrm{X}_{4} \mathrm{Y}$ ), (3) Psilochorus (Modisiminae, X0) (Additional file 29: Fig. S23), (4) the common ancestor of Crossopriza lyoni and Holocnemus hispanicus (Smeringopinae, X0) (Additional file 30: Fig. S24), and (5) during early evolution of pholcines $\left(\mathrm{X}_{1} \mathrm{X}_{2} \mathrm{Y}\right)$ (Additional file 31: Fig. S25). In modisimines, NOR data are available for Psilochorus only. Therefore, their sex chromosome-linked NORs could have arisen earlier than during the evolution of this genus. Sex-chromosome linked NORs of pholcids always have a terminal location, which suggests an origin of these structures by ectopic recombination.

Most pholcids with the X0 system and sex-chromosome-linked NORs display two terminal NORs (e.g., Physocyclus, Psilochorus) [this study]. The second NOR was probably formed by ectopic recombination between the arms of the $\mathrm{X}$ chromosome, which was facilitated by their meiotic association. Although the Physocyclus species analysed in our paper exhibit two sex chromosome-linked NORs, a study on P. globosus did not reveal these structures [30]. Since these authors used silver staining and gonial mitoses to detect NORs, it is possible that sex-chromosome linked NORs of this species are inactivated in the germline cells. Alternatively, P. globosus may belong to a Physocyclus lineage that lacks sex chromosome-linked NORs.

We revealed the most complex evolution of sex chromosome-linked NORs in the $\mathrm{X}_{1} \mathrm{X}_{2} \mathrm{Y}$ system of pholcines. According to our data, sex chromosomelinked NORs already appeared during the early evolution of pholcines, no later than before the separation of the clade containing Aetana. Paleontological [60] and molecular data [61] indicate relative antiquity of the pholcine lineage with sex chromosome-linked NORs (further SCL-NOR clade). According to the latter dataset, this clade evolved at the latest during the Jurassic. The ancestral pattern of sex chromosomelinked NORs was probably formed by a single terminal locus on the $\mathrm{X}_{1}$ chromosome, which is retained in Aetana. In another early-diverging pholcine, Belisana, the $\mathrm{X}_{1} \mathrm{X}_{2} \mathrm{Y}$ system has been transformed to $\mathrm{X} 0$. During this transformation, the terminally positioned sex chromomosome-linked NOR originating from the $\mathrm{X}_{1}$ chromosome was probably retained. The second NOR of Belisana was probably formed by ectopic recombination between the arms of the single $\mathrm{X}$ chromosome.

The subsequent evolution of NORs in the pholcine $\mathrm{X}_{1} \mathrm{X}_{2} \mathrm{Y}$ system probably involved gradual spreading of the NORs by ectopic recombination to the other $\mathrm{X}_{1}$ end, and after that to one or both ends of the $\mathrm{X}_{2}$ chromosome. Remarkably, the $\mathrm{X}$ chromosome ends are attached in male meiosis to ensure sex chromosome pairing, which could promote spreading of NORs among sex chromosomes by ectopic recombination. We suppose that spreading of NORs by ectopic recombination could also be facilitated by the association of $\mathrm{X}$ chromosome bivalents during female meiosis of spiders [5]. Besides pholcines, multiple SCS containing NORs have been found only in the ninetine Kambiwa. In contrast to pholcines, the end of the $\mathrm{X}$ chromosome containing NOR is not involved in chromosome pairing in this spider (see "Sex chromosome behaviour", p. 26).

As demonstrated in some animals, sex chromosomelinked NORs can ensure achiasmatic pairing of sex chromosomes $[62,63]$. The specific terminal location of sex chromosome-linked NORs in pholcines suggests involvement of these structures in achiasmatic pairing as well. Pairing ensured by NORs could be more stable, which may have promoted an increase in $\mathrm{Y}$ chromosome size during the evolution of pholcines. We suppose that the terminal NOR(s) of pholcids with the X0 system have a similar function as in the $\mathrm{X}_{1} \mathrm{X}_{2} \mathrm{Y}$ system, i.e. they could 
strengthen the association of the $\mathrm{X}$ chromosome arms during male meiosis.

Remarkably, our data suggest the loss or degeneration of sex chromosome-linked NORs in some pholcids, especially in the SCL-NOR clade. The most obvious cases are Pholcus pagbilao and the clade formed by Cantikus and Micropholcus. In contrast to Pholcus phalangioides, we did not detect the target $18 \mathrm{~S}$ rDNA motif on the sex chromosomes of $P$. pagbilao. This sequence could be degenerated or lost during the evolution of NORs involved in pairing. A similar pattern was found in some Drosophila species, in which most rDNA of sex chromosome-linked NOR is lost, except for motifs involved in pairing [64]. Loss/degeneration of sex chromosomelinked NORs in the common ancestor of the pholcines Cantikus and Micropholcus was accompanied by a considerable decrease of NCPs and formation of the X0 system in this lineage.

Some authors propose that sex chromosome-linked NORs could be a synapomorphy of some haplogyne clades $[9,30]$. Our data support another scenario, namely multiple invasions of NORs on sex chromosomes of haplogynes by ectopic recombinations. Although ectopic recombination is probably also a frequent mechanism of NOR dispersion in entelegynes [65], these spiders exhibit a much lower frequency of sex chromosome-linked NORs than haplogynes [5]. It was suggested that the dispersion of NORs on spider sex chromosomes by ectopic recombination is reduced by sex chromosome inactivation in meiosis of both spider sexes [65]. Since sex chromosomes of haplogynes show a similar pattern of meiotic condensation and heterochromatinisation as entelegynes [ 9 , this study], it would be interesting to determine the mechanisms facilitating the transmission of NORs on haplogyne sex chromosomes. It could be the lower degree of $\mathrm{X}$ chromosome condensation during some periods of male prophase I [this study]. In keeping with this hypothesis, NORs have almost never been detected on the $\mathrm{Y}$ chromosome of the haplogyne $\mathrm{X}_{1} \mathrm{X}_{2} \mathrm{Y}$ system [9, this study], which could be a consequence of its considerable condensation in the germline. Another possibility of frequent spreading of NORs on sex chromosomes of haplogynes could be the presence of NOR on their CSCP. Chromosomes of the CSCP are associated with the other sex chromosomes during male meiosis [7], which could facilitate spreading of NORs on these sex chromosomes.

\section{Chromosome behaviour in the male germline Modifications of meiotic division}

The prophase of the first meiotic division is modified in pholcid males. Following pachytene, nuclei enter the so-called diffuse stage. The male diffuse stage was also found in other haplogynes, in the protoentelegyne family
Leptonetidae [9], and in some clades of early-diverging mygalomorphs [6] and entelegynes [66]. The diffuse stage evolved in other animal groups as well. In some pholcids, the diplotene is reduced: recondensed bivalents exhibit a diakinetic morphology after the diffuse stage [this study]. This pattern has also been found in some other haplogynes $[9,67]$.

Pholcid karyotypes are predominated by biarmed chromosomes, which generally form more chiasmata than monoarmed ones [68]. In spite of this, most pholcids show a very low chiasma frequency in male meiosis (Additional file 2: Table S2, Additional file 7: Table S3, Additional file 12: Table S4, Additional file 19: Table S5). This pattern is probably an ancestral pholcid feature. The frequency of chiasmata is increased particularly in some pholcids with a low diploid number, namely some artemines, modisimines, and pholcines [25, 26, this study]. Since a reduction of diploid number is accompanied by decrease of total chiasma number per karyotype, increase of chiasma frequency per bivalent could be a compensatory mechanism to avoid reduction of genetic variability in these species. Pholcids usually exhibit a predomination of intercalar and distal chiasmata. A different pattern has been found in Modisimus and Physocyclus, which show a relatively high proportion of pericentric chiasmata. Relocation of chiasmata in these spiders could be a consequence of inversions, which can change the position of chiasmata [69].

\section{Polyploid cells}

Germline cells of spider males form spermiocysts, whose cells are connected by cytoplasmic bridges and synchronized during their development [70]. As a result, plates belonging to the same mitotic or meiotic phase are frequently fused on chromosome preparations [26, 30]. Besides these artefacts resembling polyploid plates, preparations of pholcid testes contained many large endopolyploid nuclei, which could belong to a specific cell lineage. Occurrence of these nuclei in testes reflects a high metabolic activity of testicular tissues. Overcondensation of sex chromosomes in endopolyploid nuclei of some pholcids could reflect transcriptional repression of these chromosomes. Endopolyploid nuclei were also reported in the testes of some other opisthothele spiders $[5,6,71]$. In pholcids, they have only been reported in the silk and venom glands so far [72].

\section{Sex chromosome behaviour}

Spider sex chromosomes are distinguished by a specific behaviour in the male germline [5, 6, 18, 38, 45, 73], which is sometimes already initiated at spermatogonial mitosis [5]. In some pholcids, the sex chromosomes exhibit a specific condensation during this division. 
Chromosomes of the $\mathrm{X}_{1} \mathrm{X}_{2} \mathrm{Y}$ system are usually associated in spermatogonia, whereas $\mathrm{X}$ chromosomes are often arranged in parallel [this study]. Moreover, sex chromosomes of this system are preferentially located in the middle of spermatogonial metaphases [9, this study]. Concerning the other pholcid sex chromosome systems, we were not able to determine the relative position of their chromosomes in spermatogonia. Notably, multiple $\mathrm{X}$ chromosomes of some entelegynes also show specific condensation, and similar arrangement and location at spermatogonia, as found in the $\mathrm{X}_{1} \mathrm{X}_{2} \mathrm{Y}$ system [5].

Despite the considerable diversity of SCS in pholcids, pairing of their sex chromosomes during male meiosis is conservative. Similar to other spiders [5], it is already established during premeiotic interphase [ 9 , this study]. Pairing of chromosomes of the $\mathrm{X}_{1} \mathrm{X}_{2} \mathrm{Y}$ system is initiated by the parallel attachment of $\mathrm{X}$ chromosomes [this study]. The mode of chromosome pairing within the pholcid $\mathrm{X}_{1} \mathrm{X}_{2} \mathrm{Y}$ system is the same as in other haplogynes [9]. Biarmed chromosomes of this system pair without chiasmata, namely by the ends of both arms $[9$, this study]. This sex chromosome attachment is probably an ancestral mode of achiasmatic sex chromosome pairing in spiders [5]. It is retained in achiasmatic XY and multiple X chromosome systems of pholcids [this study]. Remarkably, pairing of the monoarmed $\mathrm{X}_{2}$ chromosome is modified in some pholcids. In Artema $\left(\mathrm{X}_{1} \mathrm{X}_{2} \mathrm{Y}\right)$ and smeringopines $\left(\mathrm{X}_{1} \mathrm{X}_{2} \mathrm{O}\right)$, the monoarmed $\mathrm{X}_{2}$ shows standard pairing. However, attachment of the short arm is less stable in the latter group during late prophase and metaphase I. In some pholcines with monoarmed $\mathrm{X}_{2}$, only the long arm of the $\mathrm{X}_{2}$ takes part in pairing during this period [9, this study]. In contrast, there are pholcines with monoarmed $\mathrm{X}_{2}$, both ends of which participate in pairing. This pattern indicates that restriction of pairing to the long arm of $\mathrm{X}_{2}$ does not depend only on the morphology of this chromosome. Concerning the X0 system, arms of the sex chromosome are associated together in pholcids during late prophase and metaphase I [this study]. This pattern is also common in other spiders exhibiting the X0 system and biarmed sex chromosome [5, 9].

Our data suggest a possible pattern of sex chromosome pairing in the $\mathrm{X}_{1} \mathrm{X}_{2} \mathrm{X}_{3} \mathrm{X}_{4} \mathrm{Y}$ system of Kambiwa. As indicated above, two of its $\mathrm{X}$ chromosomes are probably biarmed and two other X are monoarmed. X chromosomes of Kambiwa probably pair by the ends of their arms during male meiosis. According to our model (Fig. 17), both arms of the biarmed $\mathrm{X}$ chromosomes are involved in pairing. One monoarmed $\mathrm{X}$ chromosome bears a NOR at the end, which is not involved in pairing. Since this NOR is placed in the short arm, only the long arm of this chromosome is involved in pairing. We hypothesize the same mode of pairing for the

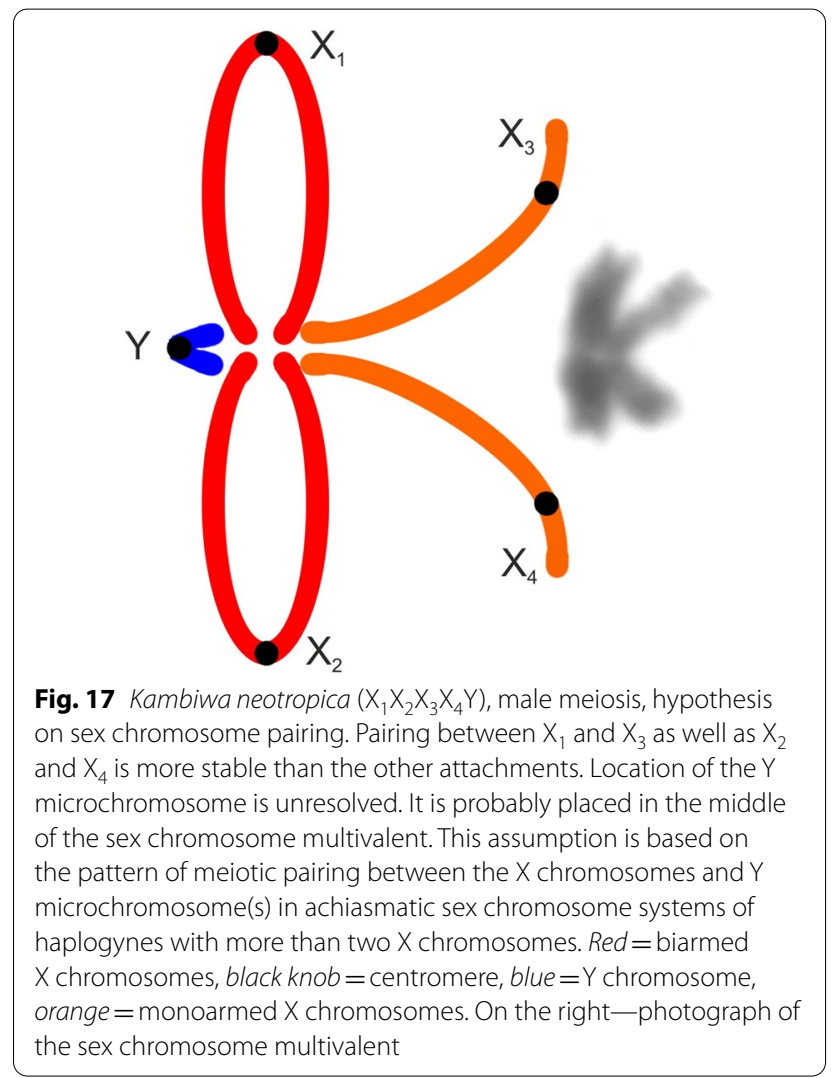

second monoarmed X chromosome. Our data indicate that the sex chromosome multivalent of Kambiwa contains two X chromosome pairs; each of them consists of one biarmed and one monoarmed chromosome. Pairing between these pairs is less stable than within them. We assume a similar location of the $\mathrm{Y}$ chromosome as in the achiasmatic sex chromosome systems of other haplogynes with more than two X [14], namely in the middle of the multivalent.

Meiotic segregation of the sex chromosomes is modified in pholcid males. Regardless of the type of SCS, it is usually delayed in anaphase I. The sex chromosome(s) of some pholcids show precocious or delayed division during metaphase II. In some pholcids, X chromosome segregation is also delayed during the second meiotic division [this study].

As in other spiders, the sex chromosomes of pholcids are usually placed at the periphery of the plate during premeiotic interphase, prophase and metaphase I. Notably, our data suggest relocation of sex chromosomes to the middle of the plate during late prophase I in pholcids with multiple X systems. The same behaviour of X chromosomes has evolved in males of avicularioid mygalomorphs [6]. Its function is unclear. After segregation during anaphase I, X chromosomes of 
pholcids retain their association until the end of meiosis, whereas the $\mathrm{Y}$ chromosome tends to be placed in the middle of the plate [this study].

As in other spiders, the course of sex chromosome condensation and pycnosis is complicated and species-specific in male meiosis of pholcids. The $\mathrm{Y}$ chromosome is often more condensed than the $\mathrm{X}$ chromosomes. In some pholcids, condensation of the $\mathrm{X}$ chromosomes is delayed during diplotene and diakinesis (e.g. Nipisa, Leptopholcus, most Pholcus species) [this study].

It should be underlined that chromosomes of the CSCP can also possess a specific behaviour in the male germline of spiders. In some mygalomorphs, they are associated and exhibit precocious chromatid separation in spermatogonial mitosis. Furthermore, they are heterochromatic in some male meiotic phases of some haplogynes and mygalomorphs $[5,6,9]$. Therefore, a large heterochromatic pair observed in male prophase I of several pholcids is probably a CSCP [this study].

\section{Phylogenetic implications}

Despite the relatively limited sample of studied species, our study emphasizes the potential of karyotype data as an independent source of information for phylogenetic reconstruction. Based on character mapping, many chromosome features were identified as apomorphies, which can be potentially used to reconstruct pholcid phylogeny. Most of these features concern the number of chromosome pairs, chromosome morphology, SCS, and NOR pattern. Character mapping also suggests, however, a high level of homoplasy and many characters that need to be mapped on terminal branches, especially those concerning chromosome morphology. In general, this suggests a limited use of certain karyotype data for the reconstruction of pholcid phylogeny. However, numerous clades established on the basis of morphological and/or molecular data are in fact supported by karyotype data:

- At the level of subfamilies: e.g. Smeringopinae (2 characters) and Pholcinae (3 characters), sister relationship of Smeringopinae and Pholcinae (2 characters) (Figs. 16, 18).

- At the level of genus-groups, e.g.:

- Sister-group relationship between Quamtana and the Pholcus group of genera (three characters) (first proposed based on morphology [76], later supported by molecular data [20]) (Fig. 18).

- Clade formed by all artemine genera except Artema (in our sample Chisosa, Wugigarra, Holocneminus, and Physocyclus) (1 character) (Fig. 15).

- Separation of Stygopholcus and Hoplopholcus (Fig. 16) (only character 15 supports a close rela- tionship between the two genera, as originally claimed e.g. by Brignoli [75-77]).

- Sister group relationship between Cantikus (previously in Pholcus) and Micropholcus (three characters) (strongly supported by molecular data, [20]) (Fig. 18).

- At the level of genera: e.g., Artema (three characters), Hoplopholcus (two characters) (Figs. 15, 16).

In some cases, the karyotype data suggest plausible alternative topologies that should be tested by molecular (ideally phylogenomic) approaches.

- Position of Ninetinae. Previous hypotheses placed ninetines either as sister to all other pholcids $[61,78]$ or together with Artema as sister to all other pholcids [20]. Ancestral ninetines probably exhibited 13 chromosome pairs, as found in Pholcophora. The ancestor of another studied ninetine, Kambiwa (12 pairs), probably had the same NCPs as Pholcophora; one pair has most probably been incorporated into the SCS. Mapping of chromosome data on the molecular tree suggests two chromosome changes in the ancestors of Ninetinae (decrease of NCPs from 15 to 13 and increase of NOR number to two loci) and the same two changes in the common ancestor of Smeringopinae + Pholcinae (Figs. 15, 16). Our chromosome data thus suggest a sister-group relationship between Ninetinae and Smeringopinae + Pholcinae. Clearly, the position of ninetines continues to be unclear.

- Phylogeny of artemines with low diploid numbers. The molecular phylogeny suggests the following topology: Chisosa (Physocyclus (Wugigarra + Holocneminus)) [21]. In this clade, the X0 system could either arise once, with subsequent reversion to the $\mathrm{XY}$ system (in Wugigarra), or it possibly evolved three times from the XY system, namely in Chisosa, Holocneminus, and Physocyclus (Fig. 15, Additional file 29: Fig. S23). The first hypothesis is improbable, since the $\mathrm{X}$ and $\mathrm{Y}$ chromosomes of Wugigarra exhibit the same mode of meiotic pairing as the chromosomes of the ancestral pholcid $\mathrm{X}_{1} \mathrm{X}_{2} \mathrm{Y}$ system. This mode of pairing would probably not be retained, if a reversion to the XY system had occurred. Although the second hypothesis (multiple convergent origin of the $\mathrm{X} 0$ system from the $\mathrm{XY}$ system) is supported by several independent origins of the $\mathrm{X} 0$ system in other pholcids, karyotype data lend some support to an alternative topology (Wugigarra (Chisosa (Holocneminus + Physocyclus))), which includes only a single origin of the $\mathrm{X} 0$ system from the $\mathrm{XY}$ system in 


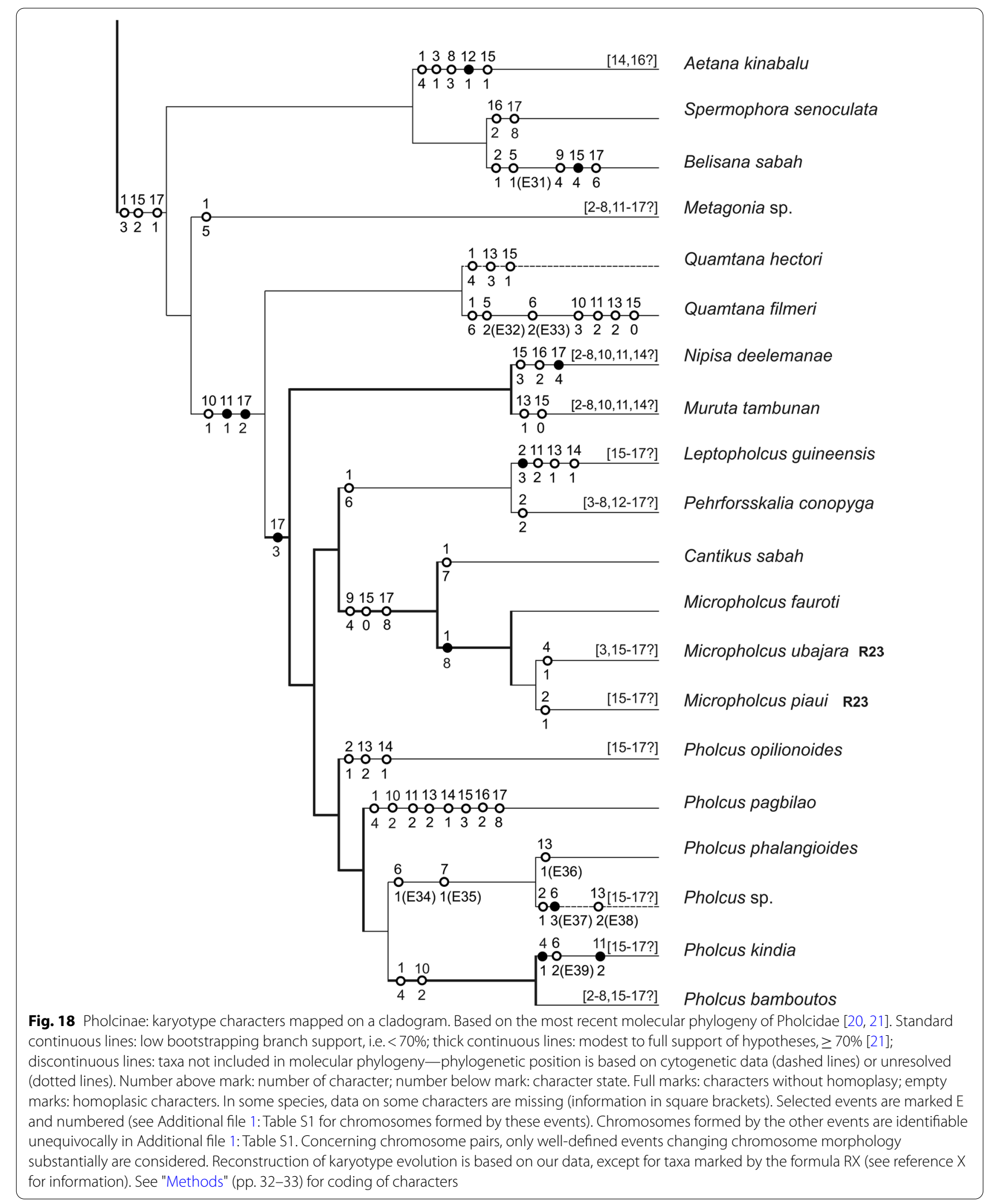


the clade, namely at the base of the clade formed by the genera Chisosa, Holocneminus, and Physocyclus. This scenario contradicts a fairly strong node linking Wugigarra and Holocneminus (bootstrapping support 81) [21], but the alternative topology supported by karyotype data should be further tested too.

- Position of Spermophora. This spider differs from most other pholcines by the absence of sex chromosome-linked NORs. According to the molecular phylogeny, Spermophora belongs to an early-diverging clade of pholcines, which also includes the genera Aetana and Belisana [21], both of which have sex chromosome-linked NORs (Fig. 18, Additional file 31: Fig. S25). In this phylogeny, the absence of sex chromosome-linked NORs in Spermophora reflects NOR loss. An alternative hypothesis, based on chromosomes, is that the absence of sex chromosomelinked NORs in Spermophora represents a symplesiomorphy of pholcids rather than a loss, suggesting a sister-group relationship between Spermophora and all other pholcines. This is a plausible scenario because the position of Spermophora (and also that of Aetana and Belisana) was not strongly resolved in the molecular phylogeny.

- Phylogeny of the CHS clade (formed by Crossopriza, Holocnemus, and Stygopholcus). The molecular phylogeny suggests the topology $H$. hispanicus (Stygopholcus ( $H$. caudatus + Crossopriza))) (Fig. 16). Karyotype data show a specific mediumsized submetacentric pair (feature 6-1) in $H$. caudatus and H. hispanicus, which evolved either once or twice. This uncertainty is related to the question of Holocnemus monophyly: H. caudatus and H. hispanicus might be sister taxa, sharing feature 6-1. Molecular data did not support such a relationship, but also did not strongly contradict it [20]. The two species are geographic neighbors and are morphologically similar, which lends further credibility to the karyotype data. The type species of Holocnemus, H. pluchei, is a morphologically isolated species and probably not closely related to $H$. caudatus and $H$. hispanicus (B.A. Huber, unpublished data). Chromosome data suggest it is probably sister taxon to the other taxa of the CHS clade (Fig. 16). Mapping of the chromosome information on a molecular cladogram suggests that eleven CPs is a synapomorphy of Stygopholcus, Holocnemus caudatus, $H$. hispanicus, and Crossopriza (Fig. 16). The molecular cladogram also suggests another synapomorphy for this clade, namely a sex chromosome-linked NOR. This structure is already present at the base of this clade in $H$. hispanicus, i.e. before the separation of Stygopholcus (Fig. 16). In contrast to $H$. hispanicus, however, Stygopholcus does not exhibit sex chrososome-linked NOR, indicating secondary loss of this marker. The chromosome data suggest a more plausible explanation of the pattern found in Stygopholcus. Similar to Spermophora, the absence of sex chromosome-linked NOR may represent a symplesiomorphy of pholcids rather than a loss, which suggests a sister-group relationship between Stygopholcus and a clade formed by $H$. caudatus, $H$. hispanicus, and Crossopriza.

- Smeringopus phylogeny. Molecular data suggested the topology S. pallidus (S. cylindrogaster (S. atomarius (S. similis + S. peregrinus))) (Fig. 16) (accepting that $S$. peregrinus, which was not included in the analysis, and S. peregrinoides are closely related). Mapping of chromosome data on this cladogram suggests two independent origins of the specific shortest chromosome pair exhibiting an acrocentric morphology, namely in S. cylindrogaster and S. atomarius. This pair might arise by pericentric inversion from a short subtelocentric pair, which is a synapomorphy of $S$. similis and S. peregrinus. Chromosome data indicate that the shortest chromosome pair exhibiting an acrocentric morphology may be a synapomorphy of $S$. cylindrogaster and $S$. atomarius. This is in agreement with a morphological cladistic analysis [79], suggesting that the sistergroup relationship between $S$. cylindrogaster and $S$. atomarius indicated by the karyotype data is a plausible alternative to the molecular hypothesis.

- Position of Pholcus phalangioides. Mapping of chromosome information on the cladogram derived from molecular data suggests an independent decrease of NCPs from eleven to ten and a considerable decrease of $\mathrm{X}_{2}$ size in the two Pholcus clades, namely P. pagbilao and African Pholcus species (Fig. 18). Chromosomes suggest an alternative topology, namely a single origin of these features at the base of a clade formed by African and South Asian members of Pholcus. A closer relationship of African Pholcus with the Southeast Asian P. pagbilao than with P. phalangioides is plausible, as the latter was identified as a rougue taxon in [20], and is thus likely misplaced in Fig. 18.

\section{Conclusions}

Our study focused on the chromosome evolution of pholcids. A high species diversity of these spiders makes them a suitable model to analyse the fundamental trends of karyotype differentiation in haplogyne araneomorphs, a large spider clade comprising 20 families and more than 6000 species. Although the karyotype evolution of 
haplogynes includes many specific traits, it is not satisfactorily understood.

As in most other haplogynes, pholcids exhibit low to modest diploid numbers $\left(2 n 0^{*}=9-33\right)$. The diploid number of Micropholcus $\left(2 \mathrm{no}^{\star}=9\right)$ is the lowest found in araneomorph spiders with monocentric chromosomes so far. Pholcid karyotypes are predominated by metacentric chromosomes, which is another typical haplogyne feature. The evolution of pholcid chromosome pairs included frequent centric fusions, pericentric inversions, and translocations. Pholcid SCS are diversified. The ancestral sex chromosome determination of haplogynes, including pholcids, is presumably the $\mathrm{X}_{1} \mathrm{X}_{2} \mathrm{Y}$ system, which exhibits a specific chromosome morphology and achiasmatic pairing in male meiosis. The chromosomes of this system are already associated during spermatogonial mitoses. Chromosomes of the $\mathrm{X}_{1} \mathrm{X}_{2} \mathrm{Y}$ system differ in the pattern of their evolution. In some pholcid lineages, the $\mathrm{X}_{1} \mathrm{X}_{2} \mathrm{Y}$ system is converted into multiple $\mathrm{X}$ or $\mathrm{XY}$ systems, which retain the original achiasmatic sex chromosome pairing, and subsequently into the X0 system. Our data also suggest frequent integration of autosome fragments into sex chromosomes, as well as inversions and translocations of these chromosomes. Evolution of some pholcine $\mathrm{X}_{1} \mathrm{X}_{2} \mathrm{Y}$ lineages has included an enormous increase in size of the $\mathrm{Y}$ chromosome. Concerning haplogynes, there are no SCS formed by rearrangements between chromosome pair(s) and achiasmatic sex chromosomes, except for Kambiwa, which indicates potent constraints preventing these events. Our study also provides a first analysis of NOR evolution within a spider family. Pholcids display a considerable diversity of NOR patterns. The ancestral pattern was probably formed by a single terminal NOR locus. The subsequent evolution was accompanied by multiple increases of NOR number, as well as multiple invasions of NORs on sex chromosomes. Almost all NORs display a terminal location, which probably reflects their preferential spreading by ectopic recombination. The $\mathrm{X}_{1} \mathrm{X}_{2} \mathrm{Y}$ system of pholcines shows a specific distribution of sex chromosome-linked NORs, which are located almost exclusively at X chromosome ends involved in pairing. This pattern indicates involvement of NORs in achiasmatic pairing. In some pholcines these NORs were lost or degenerated. As in other haplogynes, the prophase of the first meiotic division is modified in pholcid males. It includes a diffuse stage, which is distinguished by an extreme decondensation of bivalents. Another specific feature of male meiosis is a very low recombination frequency in most pholcids.

Our study suggests a very low intraspecific diversity of pholcid karyotypes. Closely related species differ, however, often by the morphology of several chromosome pairs and sex chromosomes, as well as NOR pattern.
Based on this pattern, we suppose that these changes can already appear during intraspecific karyotype differentiation. In several species, an intraspecific diversity of NOR pattern is suggested by the occurrence of heterozygotes for NOR. Rearrangements of chromosome pairs and sex chromosomes probably contribute towards the formation of interspecific reproductive barriers. Rapid sex chromosome evolution of pholcids may accelerate species diversification of these spiders. Although some karyotype features of pholcids are highly homoplasic, others are congruent with previous phylogenetic hypotheses based on morphology and/or molecules, suggesting a considerable potential for phylogenetic reconstruction. In some cases, our karyotype data suggest plausible alternative hypotheses on pholcid phylogenetic relationships, which can be tested by approaches of molecular phylogeny and phylogenomics. Our study offers novel hypotheses on karyotype evolution of haplogyne spiders, which can be tested by methods of molecular biology and cytogenetics in pholcids, as well as other haplogyne clades. Pholcids seem to be a suitable model group to analyse (1) participation of NORs in achiasmatic pairing, (2) co-evolution of sex chromosomes and NORs, (3) evolutionary differentiation of SCS composed of non-recombining chromosomes, and (4) increase and rejuvenation of the $\mathrm{Y}$ chromosome during evolution.

\section{Methods \\ Material}

In total, 197 specimens belonging to 47 pholcid species were analysed. Information on the studied species (number of analysed specimens, their sex, ontogenetic stage, and locality data) and deposition of voucher specimens are given in Additional file 32: Table S6.

\section{Preparation of Giemsa-stained slides and their evaluation} Chromosome plates were obtained from subadult or adult males, specifically from testes or the whole content of the abdomen (in small species). Slides were prepared by the spreading technique [80]. Tissues were hypotonized in $0.075 \mathrm{M} \mathrm{KCl}$ for $20-25 \mathrm{~min}$ at room temperature (RT) and fixed twice (10 and $20 \mathrm{~min})$ in ethanol: acetic acid (3:1) (RT). The cell suspension was prepared from a piece of fixed tissue in a drop of $60 \%$ acetic acid on a slide using a pair of tungsten needles. The preparation was placed on a histological plate $\left(40{ }^{\circ} \mathrm{C}\right)$. The drop was moved by a tungsten needle until almost complete evaporated. The remaining suspension was discarded. Slides were stained using 5\% Giemsa solution in Sörensen buffer (pH 6.8) for $28 \mathrm{~min}$ (RT).

Preparations were inspected under an Olympus BX 50 microscope equipped with DP 71 CCD camera. To construct the karyotype, in most cases several metaphases 
II or mitotic metaphases (preferably five), were analysed to determine the relative chromosome length ( $\mathrm{RCL}$ ) and chromosome morphology. In the case of metaphases II, plates containing both sister cells were evaluated, except for Aetana, Artema nephilit, Hoplopholcus labyrinthi, and Quamtana filmeri. In these species, only single metaphase II cells were available. In some species, determination of chromosome morphology was difficult due to chromosome coiling. In most cases, it was impossible to distinguish the CSCP from autosomes. Therefore, the CSCP and autosomes are referred to collectively as chromosome pairs. Relative chromosome length was estimated as a percentage of the total chromosome length of the haploid set (TCL). Chromosome morphology was based on the position of the centromere [81], which was calculated as the ratio of the longer and shorter chromosome arms. Based on this ratio, four chromosome morphologies were recognized, specifically metacentric (1.0-1.7), submetacentric (1.71-3.0), subtelocentric (3.01-7.0), and acrocentric ( $>7)$. Some chromosomes exhibited a transitional morphology between two of these types. In these cases, chromosome morphology was denoted by the formula $x / y$ (this formula means transition between morphology $\mathrm{x}$ and $\mathrm{y}$ ). Mediocentric chromosomes (1.0-1.3) were considered as a subset of metacentric chromosomes. Metacentric and submetacentric chromosomes were considered as biarmed, and subtelocentric and acrocentric chromosomes as monoarmed. The sex chromosome system was usually identified from meiosis of the heterogametic sex, based on segregation of the sex chromosomes and/or their behavior in prophase and metaphase I. In Psilochorus californiae, the sex chromosome system was determined on the basis of the sex chromosome-specific pattern of NORs. Some karyotypes contained several different $\mathrm{X}$ chromosomes. In this case, $\mathrm{X}$ chromosomes were numbered according to decreasing size. The preferential position of sex chromosomes was evaluated from 10 or 20 chromosome plates. Chromosomes were measured using the programme IMAGEJ 1.47 (http://imagej.nih.gov/ij/). Karyotypes were assembled using the programme Corel Photo Paint X3. In some ninetines (Kambiwa) and pholcines (Metagonia, Muruta, Nipisa, Pehrforsskalia, Pholcus bamboutos), data on chromosome morphology were not sufficient to assemble the karyotype. Karyotypes of Holocnemus caudatus, Pholcus phalangioides, and Spermophora senoculata have already been published in another paper [9]. Finally, several late prophases I (preferably five or ten) were analysed to determine the frequency of chiasmata, which was assessed as a ratio of total chiasmata number found to total bivalent number counted in the analysed plates.

\section{Detection of nucleolus organizer regions}

Nucleolus organizer regions were detected using the biotin labelled $18 \mathrm{~S}$ rDNA probe. Initially, we tested probes from several spider species. The probe from the haplogyne spider Dysdera erythrina (Dysderidae) showed the lowest level of non-specific hybridization and was used in our study. The probe was generated following [65]. The D. erythrina $18 \mathrm{~S}$ rDNA amplicon was about $1600 \mathrm{bp}$ long. It was cloned into the P-Gem T easy vector (Promega, Madison, WI, USA) and Sanger sequenced using both specific [65] and M13 universal primers. We recovered a 1549 bp long sequence, which was deposited in GenBank under the acc. no. MT886274. Blastn search revealed that the sequence partially matches the $18 \mathrm{~S}$ rDNA sequences of other D. erythrina isolates (Acc. nos. KF929034 and KY016439) with identity $>99.9 \%$. The probe was detected by streptavidin-Cy3, with amplification of the signal (biotinylated antistreptavidin, streptavidin-Cy3). Chromosomes were counterstained by DAPI (see [65] for details of method). Besides unstained slides, preparations stained by Giemsa were used for NOR detection after their observation under immersion oil objective. Immersion oil was removed from these preparations by xylene and benzine baths ( 1 min each, RT). The stain was removed from slides by their incubation in fixative (3 min, RT). In Artema, pre-treatment of slides with proteinase $\mathrm{K}$ was carried out to remove cytoplasmatic residues (see [65] for details of treatment). Selected chromosome plates were captured with an Olympus IX81 microscope equipped with an ORCA-AG CCD camera (Hammatsu), or Zeiss Axioplan 2 microscope along with F-View CCD camera (Olympus). Images were pseudocoloured (red for $\mathrm{Cy} 3$, blue for DAPI) and superimposed with $\mathrm{Cell}^{\wedge} \mathrm{R}$ software (Olympus Soft Imaging Solutions) or AnalySIS 3.2 software (Soft Imaging, System GmbH, Münster, Germany). Morphology of NOR-bearing pairs was determined using images or inferred from data on the morphology of the chromosome pairs (in case all pairs had the same morphology, e.g. metacentric). In pholcines exhibiting the $\mathrm{X}_{1} \mathrm{X}_{2} \mathrm{Y}$ system, NOR-bearing sex chromosomes were identified by a combination of two approaches. First, sex chromosomes often exhibited more intensive fluorescence than the other chromosomes during mitosis. Second, from comparison of late prophase/metaphase I and mitotic metaphase, it is possible to distinguish the NOR-bearing chromosomes of the $\mathrm{X}_{1} \mathrm{X}_{2} \mathrm{Y}$ system. In meiosis, these chromosomes pair by their NOR-bearing regions, thus forming one strong signal comprised of several NORs. 


\section{Evolution of cytogenetic characters}

We organized the obtained data into 17 characters describing the number, size, and morphology of chromosomes, SCS, and NORs. The evolution of cytogenetic characters was reconstructed by character mapping on the latest molecular phylogeny of Pholcidae [20,21] (Figs. 15, 16, 18, Additional file 29: Fig. S23, Additional file 30: Fig. S24, Additional file 31: Fig. S25). To reconstruct karyotype evolution of pholcids, we also used data obtained by other authors (see Additional file 2: Table S2, Additional file 7: Table S3, Additional file 12: Table S4, Additional file 19: Table S5). Doubtful data were excluded from the reconstruction (see "Discussion" for their analysis). The cytogenetic characters used in the reconstruction of karyotype evolution are listed below.

\section{Number, size, and morphology of chromosome pairs}

Character 1. Number of chromosome pairs: (0) 15, (1) 13, (2) 12, (3) 11, (4) 10, (5) 8, (6) 7, (7) 6, (8) 4.

Character 2. Relative size of first three chromosome pairs: (0) gradual decrease in size from the first to fourth pair, (1) a considerable increase of chromosomes of the first pair (size difference between chromosome of first and second pair $>1.5 \%$ of TCL), (2) first two pairs considerably increased (size difference between chromosome of second and third pair $>1.5 \%$ of TCL), (3) first three pairs considerably increased (size difference between chromosome of third and fourth pair $>1.5 \%$ of TCL).

Character 3. Relative size of the smallest chromosome pair: (0) small size difference between last two pairs, (1) considerable reduction of last pair (size difference between chromosomes of last two pairs $>1.5 \%$ of TCL).

Character 4. Morphology of longest chromosome pair: (0) metacentric, (1) submetacentric.

Character 5. Morphology of long marker chromosome pair: (0) metacentric, (1) submetacentric, (2) subtelocentric.

Character 6. Morphology of medium-sized marker chromosome pair: (0) metacentric, (1) submetacentric, (2) subtelocentric, (3) acrocentric.

Character 7. Morphology of short marker chromosome pair: (0) metacentric, (1) submetacentric, (3) subtelocentric, (4) acrocentric.

Character 8. Morphology of shortest chromosome pair: (0) metacentric, (1) submetacentric, (2) subtelocentric, (3) acrocentric.

In the case of characters $2-8$, we did not consider chromosome pairs of species to be homologous except for several specific cases, where we consider some chromosomes of closely related species exhibiting a very similar karyotype to probably be homologous. It concerns the events E1, E2, E3, E24, E27, E28, E29, E34, and E35 (see Additional file 1: Table S1).

\section{Sex chromosomes}

Character 9. Sex chromosome system: (0) $\mathrm{X}_{1} \mathrm{X}_{2} \mathrm{Y}$, (1) XY, (2) $\mathrm{X}_{1} \mathrm{X}_{2} \mathrm{X}_{3} \mathrm{X}_{4} \mathrm{Y}$, (3) $\mathrm{X}_{1} \mathrm{X}_{2} \mathrm{O}$, (4) $\mathrm{X} 0$, (5) $\mathrm{X}_{1} \mathrm{X}_{2} \mathrm{X}_{3} \mathrm{O}$.

Character 10. $\mathrm{X}_{2}$ chromosome. (0) large element, similar to $\mathrm{X}_{1},(1)$ reduction of $\mathrm{X}_{2}$ (size $\leq 7 \%$ of TCL for Arteminae, Pholcinae; size $\leq 7.5 \%$ of TCL for Smeringopinae), (2) considerable reduction of $X_{2}$ (size $\leq 4 \%$ of TCL), (3) considerable increase of $X_{2}$ ( $\geq 2 \%$ of TCL).

Character 11. Y chromosome. (0) tiny, (1) increase (size $\geq 4.5 \%$ of TCL), (2) considerable increase (size $\geq 7 \%$ of TCL).

Character 12. $\mathrm{X}_{1}$ morphology: (0) metacentric, (1) submetacentric.

Character 13. $\mathrm{X}_{2}$ morphology: (0) metacentric, (1) submetacentric, (2) subtelocentric, (3) acrocentric.

Character 14. Y morphology: (0) metacentric, (1) submetacentric.

\section{Nucleolus organizer regions}

Character 15. Total number of NOR loci on chromosome pairs: (0) 1, (1) 2, (2) 3, (3) 4, (4) 5.

Character 16. Location of NORs on chromosome pairs: (0) each NOR-bearing pair with one terminal NOR, (1) pericentric, (2) each NOR-bearing pair with one terminal NOR, except for one pair with NOR at both ends.

Character 17. Sex chromosome-linked NORs: (0) NORs located exclusively on chromosome pairs, (1) NOR at one end of $X_{1}$ chromosome $\left(X_{1} X_{2} Y\right.$ system $),(2)$ NOR at both ends of $X_{1}$ chromosome $\left(X_{1} X_{2} Y\right.$ system), (3) NOR at both ends of $X_{1}$ chromosome plus one end of $X_{2}$ chromosome ( $\mathrm{X}_{1} \mathrm{X}_{2} \mathrm{Y}$ system), (4) NOR at both ends of $\mathrm{X}_{1}$ chromosome and $\mathrm{X}_{2}$ chromosome, as well as at one end of $Y$ chromosome $\left(X_{1} X_{2} Y\right.$ system), (5) NOR at one $X$ chromosome end (X0 system), (6) NOR at both $\mathrm{X}$ chromosome ends (X0 system), (7) X chromosome-linked NOR $\left(\mathrm{X}_{1} \mathrm{X}_{2} \mathrm{X}_{3} \mathrm{X}_{4} \mathrm{Y}\right.$ system), (8) loss of all sex chromosome-linked NORs.

\section{Abbreviations}

AT: Adenine-thymine; CAS: Czech Academy of Science; CCD: Chargecoupled device; CHS clade: Clade formed by Crossopriza, Holocnemus, and Stygopholcus; Cl: Centromeric index; CP: Chromosome pair; CSCP: Cryptic sex chromosome pair; Cy-3: Cyanomycin 3; DAPI: 4',6-Diamidine-2'-phenylindole dihydrochloride; FISH: Fluorescence in situ hybridization; $\mathrm{KCl}$ : Kalium chloride; NCPs: Number of chromosome pairs; NOR: Nucleolus organizer region; RCL: Relative chromosome length; rDNA: Ribosomal DNA; rRNA: Ribosomal RNA; RT: Room temperature; S: Svedberg unit; SCB: Sex chromosome body; SCLNOR clade: Sex chromosome-linked NOR clade (belonging to Pholcinae); SCS: Sex chromosome system; TCL: Total chromosome length; 2n: Diploid chromosome number. 


\section{Supplementary Information}

The online version contains supplementary material available at https://doi. org/10.1186/s12862-021-01750-8.

Additional file 1: Table S1. Species under study, male karyotype data (including standard deviation). Abbreviations: $\mathrm{EX}=$ product of event $\mathrm{X}$ ( $X=$ number of event), phase $=$ phase of $\mathrm{mitotic} /$ meiotic division used to obtain data on chromosome morphology ( $\mathrm{m}$ mit $=$ mitotic metaphase, $\mathrm{m}$ I = metaphase I, $\mathrm{m} \|$ = metaphase II), plates = number of chromosome plates evaluated, parameters $=$ parameters used to describe chromosome morphology $[\mathrm{Cl}=$ centromeric index, $\mathrm{RCL}=$ relative chromosome length (\% of TCL)]. Chromosome morphology is indicated by background colour of a box (pink: metacentric, brown: submetacentric, dark blue: subtelocentric, light blue: acrocentric, red: unknown).

Additional file 2: Table S2. Arteminae, summary of male cytogenetic data, including results of other authors. Doubtful data are not included. See database [24] for full list of published data on pholcid karyotypes, including doubtful data. Abbreviations: $\mathrm{a}=$ acrocentric, $\mathrm{bi}=$ biarmed, $\mathrm{CP}=$ chromosome pair, $\mathrm{m}=$ metacentric, $\mathrm{n}=$ number of plates evaluated, $\mathrm{p}=$ short chromosome arm, $\mathrm{pc}=$ pericentric, $\mathrm{q}=$ long chromosome arm, SC = sex chromosome, SCS = sex chromosome system, $\mathrm{sm}=$ submetacentric,${ }^{\circledR}=$ revision of data of other authors, st $=$ subtelocentric, $t=$ terminal, ? = unknown, ${ }^{*} X=$ data of other authors $(X=$ reference number).

Additional file 3: Fig. S1. Arteminae, Artema atlanta, male karyotype, stained by Giemsa. Based on two sister metaphases II. Chromosomes metacentric, except for four submetacentric pairs (nos $1,6,9,12)$ and subtelocentric $X_{2}$ chromosome. Note low condensation of $X$ chromosomes. Bar $=10 \mu \mathrm{m}$.

Additional file 4: Fig. S2. Sex chromosomes of artemines with the $X_{1} X_{2} Y$ and $X Y$ systems. Stained by Giemsa. $X_{1}=X_{1}$ chromosome, $X_{2}=X_{2}$ chromosome, $Y=Y$ chromosome. (a-c) Artema atlanta $\left(X_{1} X_{2} Y\right)$. a Metaphase I, composed of 15 bivalents and sex chromosome trivalent. b Metaphase II, with chromosomes $X_{1}$ and $X_{2}$ at the periphery of the plate. c Metaphase II with $Y$ chromosome. (d-f) Wugigarra sp. d Metaphase l, consisting of seven bivalents and a XY pair. e Metaphase II containing a positively heteropycnotic $X$ chromosome $(n=8)$. $\mathbf{f}$ Metaphase II, containing a $Y$ chromosome $(\mathrm{n}=8)$. $\mathrm{Bar}=10 \mu \mathrm{m}$.

Additional file 5: Fig. S3. Sex chromosomes of artemines with the $X 0$ system. Stained by Giemsa. X = X chromosome. (a, b) Chisosa diluta. a Metaphase I, consisting of six bivalents and a peripheral $X$ chromosome. b Group of metaphases II separated by lines. It consists of one metaphase containing a positively heteropycnotic X chromosome $(n=7$, in the middle of the plate) and two metaphases without sex chromosome (left metaphase is incomplete); (c, d) Holocneminus sp. c Metaphase I, comprising seven bivalents and peripheral $X$ chromosome. $\mathbf{d}$ Two sister metaphases II separated by a line ( $n=8$ including $X$ chromosome $+n=7$ ); (e, f) Physocyclus dugesi. e Diplotene, comprising seven bivalents and peripheral X chromosome. f Anaphase II. Note slight positive heteropycnosis of $\mathrm{X}$ chromosome. Bar $=10 \mu \mathrm{m}$

Additional file 6: Fig. S4. Arteminae, males, detection of NORs (FISH). Arrowhead $=$ NOR-bearing chromosome $(\mathbf{b}, \mathbf{c})$ or bivalent $(\mathbf{a}, \mathbf{d}), X=X$ chromosome, $Y=Y$ chromosome. $(\mathbf{a}, \mathbf{b})$ Artema atlanta $\left(X_{1} X_{2} Y\right)$. a Metaphase I, note NOR-bearing bivalent. b Two fused sister metaphases II, one pair of submetacentric chromosomes bears a terminal NOR; c Wugigarra sp. (XY), two fused sister metaphases II. Note chromosomes of a subtelocentric pair bearing terminal NOR at the end of short arm; $\mathbf{d}$ Chisosa diluta (X0), diffuse stage, one bivalent includes a NOR. Bar $=10 \mu \mathrm{m}$.

Additional file 7: Table S3. Modisiminae and Ninetinae, summary of male cytogenetic data, including results of other authors. Doubtful data are not included. See database [24] for full list of published data on pholcid karyotypes, including doubtful data. Abbreviations: bi = biarmed, $\mathrm{CP}=$ chromosome pair, $\mathrm{m}=$ metacentric, $\mathrm{n}=$ number of plates evaluated, $\mathrm{p}=$ short chromosome arm, $\mathrm{q}=$ long chromosome arm, $\mathrm{SC}=$ sex chromosome, $\mathrm{SCS}=$ sex chromosome system, $\mathrm{sm}=$ submetacentric, $\mathrm{st}=$ subtelocentric, $\mathrm{t}=$ terminal, $?=$ unknown, $^{*} \mathrm{X}=$ data of other authors ( $X=$ reference number).

Additional file 8: Fig. S5. Sex chromosome systems of modisimines. Stained by Giemsa. X = X chromosome. (a-c) Anopsicus sp. (X0) a metaphase $\mathrm{I}$, consisting of eight bivalents and peripheral $X$ chromosome. $\mathbf{b}$ Metaphase II, including X chromosome $(n=9)$. c Metaphase II, without $X$ chromosome $(\mathrm{n}=8)$; (d, e) Modisimus cf. elongatus (X0). $\mathbf{d}$ Diplotene, comprising eight bivalents and a peripheral $X$ chromosome. e Two sister metaphases II separated by a line $(n=8+n=9$, including peripheral $X$ chromosome); (f, g) Psilochorus pallidulus (X0). f Diplotene, comprising eight bivalents and peripheral X chromosome. $\mathbf{g}$ Two sister metaphases $\|$ ( $n=9$, including $X$ chromosome $+n=8)$. Bar $=10 \mu \mathrm{m}$.

Additional file 9: Fig. S6. Psilochorus (Modisiminae), male karyotypes, stained by Giemsa. Sex chromosome is the longest element of the karyotype. a Psilochorus californiae. Karyotype metacentric, except for two submetacentric (nos 2,7) and one subtelocentric pairs (no. 5). Based on spermatogonial metaphase, centromeres marked by arrowheads; $\mathbf{b} P$. pallidulus. Chromosomes metacentric. Based on two sister metaphases II. Bar $=10 \mu \mathrm{m}$.

Additional file10: Fig. S7. Sex chromosome systems of ninetines. Stained by Giemsa. Figures $\mathbf{a}$ and $\mathbf{b}$ contain a scheme of the multivalent. $\mathrm{M}=$ sex chromosome multivalent, $\mathrm{O}=$ overlapping of two bivalents, $X=X$ chromosome, $X_{1}=X_{1}$ chromosome, $X_{2}=X_{2}$ chromosome, $Y=Y$ chromosome. $(\mathbf{a}, \mathbf{b})$ Kambiwa neotropica $\left(X_{1} X_{2} X_{3} X_{4} Y\right)$, plates of the first meiotic division consisting of 12 bivalents plus a sex chromosome multivalent consisting of four "arms". Two "arms" are thick (red) and two are thin (orange). a Diakinesis. b Metaphase I. Note cross-shaped morphology of multivalent; (c, d) Pholcophora americana $\left(X_{1} X_{2} Y\right)$. c Diakinesis, comprising 13 bivalents and sex chromosomes $X_{1}, X_{2}$, and $Y$. Sex chromosomes show end-to-end paring. $\mathbf{d}$ Two sister metaphases II separated by a line $(n=15$, including chromosomes $X_{1}$ and $X_{2}+n=14$ including $Y$ microchromosome). Bar $=10 \mu \mathrm{m}$.

Additional file 11: Fig. S8. Cytogenetics of ninetines $(\mathbf{a}-\mathbf{c})$ and pholcines $(\mathbf{d}-\mathbf{i})$, male germline. Figures $\mathbf{d}, \mathbf{f}, \mathbf{i}$ contain scheme of sex chromosome trivalent $\mathrm{X}_{1} \mathrm{X}_{2} \mathrm{Y} . \mathrm{B}=$ large bivalent, $\mathrm{H}=$ large bivalent exhibiting positive heteropycnosis, mon $=$ monoarmed $X$ chromosome, $T$ = sex chromosome trivalent, $X=X$ chromosome, $X_{1}=X_{1}$ chromosome, $X_{2}=X_{2}$ chromosome, $Y=Y$ chromosome. (a-c) Kambiwa neotropica $\left(X_{1} X_{2} X_{3} X_{4} Y\right)$. a Spermatogonial metaphase $(2 n=29)$. Chromosomes are biarmed, except for two monoarmed chromosomes. b Metaphase II, consisting of 12 chromosomes and cluster of four positively heteropycnotic $X$ chromosomes. $\mathbf{c}$ Metaphase II, formed by 12 chromosomes and a $Y$ microchromosome; $\mathbf{d}$ Aetana kinabalu $\left(\mathrm{X}_{1} \mathrm{X}_{2} \mathrm{Y}\right)$, incomplete metaphase I. Sex chromosomes pair by ends of their arms, $X$ chromosomes are positively heteropycnotic; (e, $\mathbf{f})$ Metagonia sp. $\left(\mathrm{X}_{1} \mathrm{X}_{2} \mathrm{Y}\right)$, late prophase I. Note low chromosome condensation. e Diplotene. Plate consists of eight bivalents and almost decondensed sex chromosomes. $f$ Diakinesis, note the $X_{1} X_{2} Y$ trivalent, $Y$ chromosome more condensed than $X$ chromosomes; $(\mathbf{g}, \mathbf{h})$ Pehrforsskalia conopyga $\left(X_{1} X_{2} Y\right)$. $g$ Early diplotene. Note sex chromosomes forming a compact positively heteropycnotic body and large positively heteropycnotic bivalent. $\mathbf{h}$ Metaphase I, formed by seven bivalents and sex chromosome trivalent. Two bivalents (B) are much longer than the remaining ones. Note tiny $Y$ chromosome; $\mathbf{i}$ Pholcus bamboutos $\left(X_{1} X_{2} Y\right)$, transition from metaphase to anaphase I. Note the delayed separation of sex chromosomes. Only one end of the $X_{2}$ chromosome takes part in pairing. The $Y$ chromosome is positively heteropycnotic. Bar $=10 \mu \mathrm{m}$.

Additional file 12: Table S4. Pholcinae, summary of male cytogenetic data, including results of other authors. Doubtful data are not included. See database [24] for full list of published data on pholcid karyotypes, including doubtful data. Abbreviations: $\mathrm{a}=$ acrocentric, $\mathrm{bi}=$ biarmed, $\mathrm{CP}=$ chromosome pair, $\mathrm{m}=$ metacentric, $\mathrm{n}=$ number of plates evaluated, $\mathrm{p}=$ short chromosome arm, $\mathrm{q}=$ long chromosome arm, SC = sex chromosome, $\mathrm{SCS}=$ sex chromosome system, $\mathrm{sm}=$ submetacentric, ${ }^{\circledR}=$ revision of data of other authors, $\mathrm{st}=$ subtelocentric, $\mathrm{t}=$ terminal, $?=$ unknown, ${ }^{*} X=$ data of other authors $(X=$ reference number $)$.

Additional file 13: Fig. S9. Sex chromosomes of pholcines with the $X_{1} X_{2} Y$ system, part I. Stained by Giemsa. Figures $\mathbf{a}, \mathbf{d}$, e contain a scheme of the 
sex chromosome trivalent. $X_{1}=X_{1}$ chromosome, $X_{2}=X_{2}$ chromosome, $Y=Y$ chromosome, $P S=$ precocious separation of chromosomes of the bivalent. (a-c) Aetana kinabalu. a Metaphase I, comprising 11 bivalents (one bivalent shows a precocious separation of chromosomes) and a sex chromosome trivalent. $\mathbf{X}$ chromosomes are positively heteropycnotic. $\mathbf{b}$ Metaphase II, containing $X$ chromosomes $(n=12)$. c Transition metaphase II/anaphase II, fusion of two sister plates. Note X chromosomes exhibiting a delayed separation of chromatids and a Y microchromosome; (d-f) Nipisa deelemanae. $\mathbf{d}$ Metaphase I, comprising 11 bivalents and sex chromosome trivalent. e Part of a plate formed by several fused metaphases I, sex chromosome trivalent encircled. f Two sister metaphases II separated by a line $(n=12$, including $Y$ microchromosome $+n=13$ including metacentric chromosomes $X_{1}$ and $X_{2}$ ). Note the reduction of the $X_{2}$ chromosome. Bar $=10 \mu \mathrm{m}$.

Additional file14: Fig. S10. Sex chromosomes of pholcines with the $X_{1} X_{2} Y$ system, part II. Stained by Giemsa. Figures $\mathbf{a}, \mathbf{b}$ contain a scheme of the sex chromosome trivalent. $T=$ trivalent, $X_{1}=X_{1}$ chromosome, $X_{2}=X_{2}$ chromosome, $Y=Y$ chromosome. (a-d) Leptopholcus guineensis. a Early diplotene, consisting of seven bivalents and a sex chromosome trivalent, $X$ chromosomes exhibit a low condensation. b Two fused diplotene. $\mathbf{c}$ Metaphase II, including $X$ chromosomes $(n=9)$. $X 1$ chromosome is positively heteropycnotic. $\mathbf{d}$ Metaphase II, including a $Y$ chromosome $(n=8)$; (e, f) Metagonia sp. e Spermatogonial metaphase, note the Y microchromosome. $\mathbf{f}$ Diplotene, note the positively heteropycnotic body formed by the sex chromosomes. Bar $=10 \mu \mathrm{m}$.

Additional file15: Fig. S11. Sex chromosomes of pholcines with the $X_{1} X_{2} Y$ system, part III. Stained by Giemsa. Figures $\mathbf{a}, \mathbf{c}, \mathbf{e}$ contain a scheme of the sex chromosome trivalent. $\mathrm{c}=$ centromere, $\mathrm{X}_{1}=\mathrm{X}_{1}$ chromosome, $X_{2}=X_{2}$ chromosome, $Y=Y$ chromosome. $(\mathbf{a}, \mathbf{b})$ Muruta tambunan. $\mathbf{a}$ Diakinesis, consisting of 11 bivalents and a sex chromosome trivalent. $\mathbf{b}$ Two sister metaphases II separated by a line $(n=12$, including $Y$ chromosome $+n=13$ including chromosomes $X_{1}$ and $X_{2}$ ). Note the metacentric $\mathrm{X}_{1}$ chromosome and submetacentric $\mathrm{X}_{2}$ chromosome on the periphery of the plate. They exhibit positive heteropycnosis; (c, d) Pholcus phalangioides. c Diakinesis, comprising 11 bivalents and a sex chromosome trivalent, which is placed in the middle of the plate and exhibits positive heteropycnosis. Concerning the $X_{2}$ chromosome, only end of the long arm is involved in pairing. $\mathbf{d}$ Anaphase II. Note the positive heteropycnosis of the sex chromosomes. The X chromosomes are associated; (e, f) Spermophora senoculata. e Metaphase I, comprising 11 bivalents and a sex chromosome trivalent. $\mathbf{f}$ Metaphase II, X chromosomes are less condensed than the other chromosomes; ( $\mathbf{g}, \mathbf{h})$ Quamtana hectori. $\mathbf{g}$ Metaphase I, composed of 10 bivalents and a sex chromosome trivalent. Concerning the $X_{2}$ chromosome, only one end is involved in pairing. $\mathbf{h}$ Two sister metaphases II separated by a line $(n=11$ including $Y$ chromosome $+n=12$ including chromosomes $X_{1}$ and $X_{2}$ ). Bar $=10 \mu \mathrm{m}$.

Additional file 16: Fig. S12. Pholcinae, male karyotypes, Giemsa staining. Based on metaphase II (a) or two sister metaphases II (b-d). a Aetana kinabalu, haploid set, karyotype metacentric except for submetacentric $X_{1}$ chromosome and acrocentric chromosome (no. 10), which is considerably reduced in comparison with preceding chromosome. The Y chromosome is from another metaphase II. Morphology of the $Y$ chromosome is unresolved; b Pholcus pagbilao. Karyotype is metacentric except for three submetacentric pairs (nos $5,7,10$ ), submetacentric $Y$ chromosome, and acrocentric $\mathrm{X}_{2} ; \mathbf{c}$ P. opilionoides, chromosomes metacentric except for five submetacentric pairs (nos 2-6) and acrocentric $X_{2}$. Chromosome $X_{1}$ is the longest chromosome of karyotype. On the contrary, $Y$ is the smallest one. Y chromosome is replaced by $Y$ from another plate; $\mathbf{d}$ Quamtana hectori. Karyotype metacentric, except for acrocentric $\mathrm{X}_{2}$. Centromeres of sex chromosomes marked by arrowheads. Bar $=10 \mu \mathrm{m}$.

Additional file 17: Fig. S13. Sex chromosomes of pholcines with the XO system. Stained by Giemsa. X = X chromosome. (a, b) Belisana sabah. a Metaphase I, consisting of 11 bivalents and a peripheral $X$ chromosome. $\mathbf{b}$ Two sister metaphases II separated by a line $(n=11+n=12$, including a positively heteropycnotic $X$ chromosome); (c, d) Cantikus sabah. c Diplotene, comprising six bivalents and a positively heteropycnotic $\mathrm{X}$ chromosome placed on the periphery of the plate. $\mathbf{d}$ Prometaphase
Il including the $X$ chromosome $(n=7) ;(\mathbf{e}, \mathbf{f})$ Micropholcus fauroti. e Diplotene composed of four bivalents and a positively heteropycnotic $X$ chromosome placed on the periphery of the plate. $\mathbf{f}$ Plate formed by fusion of two sister metaphases II. It includes a negatively heteropycnotic $\mathrm{X}$ chromosome. $\mathrm{Bar}=10 \mu \mathrm{m}$.

Additional file 18: Fig. S14. Pholcinae, males, detection of NORs (FISH). Figures $\mathbf{c}$, e contain a scheme of the sex chromosome trivalent $X_{1} X_{2} Y$ (red = signal formed by several NORs). Arrowhead $=$ NOR-bearing chromosome (a, b, d, $\mathbf{f}-\mathbf{h})$, bivalent $(\mathbf{c}, \mathbf{e})$ or trivalent $(\mathbf{c}, \mathbf{e})$, open arrowhead $=$ sex chromosome-linked NOR, T = sex chromosome trivalent, $X_{1}=X_{1}$ chromosome, $X_{2}=X_{2}$ chromosome, $Y=Y$ chromosome, $+=$ signal formed by several NORs. a Micropholcus fauroti (X0), mitotic metaphase. Note association of two homologous chromosomes containing terminal NOR (in - interphase nucleus); (b, c) Nipisa deelemanae $\left(X_{1} X_{2} Y\right)$. b Mitotic metaphase. $X$ chromosomes $\left(X_{1}, X_{2}\right)$ and another chromosome (II) bear a terminal NOR at both ends. Five other chromosomes, including $Y$ chromosome, involves one terminal NOR only. The sex chromosomes $X_{1}$ and $X_{2}$ are associated in parallel in the middle of the plate. $\mathbf{b}$ Metaphase $I_{\text {, }}$ note the three bivalents bearing a NOR and the sex chromosome trivalent with a signal in the region of chromosome pairing (see scheme); (d- $\mathbf{f}$ ) Quamtana hectori $\left(X_{1} X_{2} Y\right)$. d Mitotic metaphase (separated by a line from another plate). $X_{1}$ chromosome bears two NORs, each at opposite end of the chromosome. Chromosomes of two pairs also include a terminal NOR; e Metaphase I, two bivalents contain NOR. The sex chromosome trivalent contains a signal in region of chromosome pairing (see scheme). f Plate formed by fused sister metaphases II, chromosomes of NOR-bearing pairs exhibit biarmed morphology. The $X_{1}$ chromosome is terminated by NOR at both ends. $Y$ chromosome considerably condensed, without signal; $(\mathbf{g}, \mathbf{h})$ Q. filmeri $\left(X_{1} X_{2} Y\right)$, mitotic plates. The $X_{1}$ chromosome bears two NORs, each at opposite end of chromosome. Chromosomes of one pair also contain a terminal NOR. $\mathbf{g}$ Prophase, sex chromosomes exhibit a more intensive fluorescence than the other chromosomes. $\mathbf{h}$ Metaphase. Bar $=5 \mu \mathrm{m}$ except for c, e, $\mathbf{g}(10 \mu \mathrm{m})$.

Additional file 19: Table S5. Smeringopinae, summary of male cytogenetic data, including results of other authors. Doubtful data are not included. See database [24] for full list of published data on pholcid karyotypes including doubtful data. Abbreviations: $\mathrm{a}=$ acrocentric, $\mathrm{bi}=$ biarmed, $\mathrm{CP}=$ chromosome pair, $\mathrm{m}=$ metacentric, $\mathrm{n}=$ number of plates evaluated, $p=$ short chromosome arm, $q=$ long chromosome arm, SC = sex chromosome, SCS = sex chromosome system, sm = submetacentric, ${ }^{\circledR}=$ revision of data of other authors, st = subtelocentric, $\mathrm{t}=$ terminal, ? = unknown, ${ }^{*} X=$ data of other authors $(X=$ reference number).

Additional file 20: Fig. S15. Hoplopholcus and Smeringopus (Smeringopinae), male karyotypes, stained by Giemsa. Based on metaphase II (a) or two sister metaphases II (b-e). Autosome pairs decrease gradually in size. The $X_{1}$ is the longest element of the set (except for $\mathbf{d}$ ). Karyotypes are predominated by metacentrics. a $H$. labyrinthi, haploid set, note the two submetacentric chromosomes (nos 5, 9), subtelocentric chromosome (no. 6) and subtelocentric $X_{2}$ chromosome; $\boldsymbol{b}$ S. atomarius, note one submetacentric (no. 3) and one acrocentric pairs (no. 13), and submetacentric $X_{2} ; \mathbf{c} S$. ndumo, note two submetacentric pairs (nos 4,11$) ; \mathbf{d}$ S. peregrinus, note three submetacentric (nos 1, 4,6) and one subtelocentric pairs (no. 12), and subtelocentric $X_{2}$. Sex chromosomes positively heteropycnotic; $\mathbf{e}$ Smeringopus sp., note two submetacentric pairs (nos 1, 2), one acrocentric pair (no. 10) and acrocentric $X_{2}$. Bar $=10 \mu \mathrm{m}$.

Additional file 21: Fig. S16. Crossopriza lyoni (Smeringopinae), male karyotype, Giemsa staining. Based on two sister metaphases II. Karyotype metacentric, except for submetacentric pairs nos 2 and 11. First two pairs differ from the other ones by large size. The $\mathrm{X}$ chromosome is the longest chromosome of the set. It is slightly positively heteropycnotic. Bar $=10 \mu \mathrm{m}$.

Additional file 22: Fig. S17. Sex chromosomes of smeringopines with multiple $X$ chromosomes. Stained by Giemsa. $X=X$ chromosome, $X_{1}=X_{1}$ chromosome, $X_{2}=X_{2}$ chromosome. $(\mathbf{a}, \mathbf{b})$ Hoplopholcus forskali $\left(X_{1} X_{2} 0\right)$. a Diakinesis, composed of 13 bivalents and two $X$ chromosomes, note the end-to-end association of the X chromosomes. b Telophase I, half plate 
containing X chromosomes; (c-e) Smeringopus ndumo $\left(X_{1} X_{2} 0\right)$. c Diakinesis, comprising 13 bivalents and two $X$ chromosomes. $\mathbf{d}$ Metaphase $I I$, containing $X$ chromosomes $(n=15)$. e Metaphase Il, without the seX chromosomes $(n=13) ;(\mathbf{f}-\mathbf{h})$ S. pallidus $\left(X_{1} X_{2} X_{3} 0\right)$. $\mathbf{f}$ Diakinesis, composed of 13 bivalents and three $X$ chromosomes, sex chromosomes grouped in the middle of the plate. $\mathbf{g}$ Metaphase II with $X$ chromosomes $(n=16)$. $X$ chromosomes are associated at the periphery of the plate. They exhibit a slight positive heteropycnosis. $\mathbf{h}$ Metaphase II, without sex chromosomes ( $\mathrm{n}=13)$. Bar $=10 \mu \mathrm{m}$.

Additional file 23: Fig. S18. Sex chromosomes of smeringopines with the X0 system. Stained by Giemsa. X = X chromosome. $(\mathbf{a}, \mathbf{b})$ Crossopriza Iyoni. a Metaphase I composed of 11 bivalents and X chromosome. $\mathbf{b}$ Anaphase I; (c, d) Holocnemus pluchei. c Metaphase I, consisting of 13 bivalents and an $\mathrm{X}$ chromosome. The $\mathrm{X}$ chromosome is placed at the periphery of the plate. Note the association of terminal parts of the $\mathrm{X}$ chromosome arms. d Metaphase II, including the X chromosome. This element is slightly positively heteropycnotic; (e, f) Stygopholcus skotophilus. e Diplotene, composed of 11 bivalents and an X chromosome. $\mathrm{f}$ Plate formed by two fused sister metaphases $I I, 2 n=23$. Note the positively heteropycnotic $X$ chromosome. $\mathrm{Bar}=10 \mu \mathrm{m}$.

Additional file 24: Fig. S19. Pholcidae, male germline, behaviour of sex chromosomes prior to meiosis. Arrow $=$ sex chromosomes, $X_{1}=X_{1}$ chromosome, $X_{2}=X_{2}$ chromosome, $Y=Y$ chromosome. $(\mathbf{a}, \mathbf{b})$ Muruta tambunan $\left(X_{1} X_{2} Y\right)$, mitotic metaphase, chromosomes $X_{1}, X_{2}$, and $Y$ positively heteropycnotic. Chromosomes $\mathrm{X}_{2}$ and $\mathrm{Y}$ are approximately of the same size. $\mathbf{a}$ Sex chromosomes grouped in the middle of the plate. $\mathbf{b}$ Chromosomes $X_{1}$ and $X_{2}$ associated in parallel, $Y$ chromosome released from the association; $c$ Artema nephilit $\left(X_{1} X_{2} Y\right)$, early mitotic metaphase, $X$ chromosomes are marked by a dotted line. They are associated in parallel in the middle of the plate. Their condensation is slightly delayed in comparison with the other chromosomes; $\mathbf{d}$ Hoplopholcus cecconii $\left(\mathrm{X}_{1} \mathrm{X}_{2} \mathrm{O}\right)$ transition from mitotic metaphase to anaphase. Chromosome $X_{1}$ is placed in the middle of the plate; $(\mathbf{e}, \mathbf{f})$ Pholcus kindia $\left(X_{1} X_{2} Y\right)$. e Premeiotic interphase. $X$ chromosomes pair in parallel in the middle of the nucleus. The $Y$ chromosome does not take part in pairing. $\mathbf{f}$ Preleptotene. Sex chromosomes are associated in the middle of the nucleus, the $Y$ chromosome is more condensed than the $\mathrm{X}$ chromosomes. Bar $=10 \mu \mathrm{m}$.

Additional file 25: Fig. S20. Pholcidae, male meiosis, condensation and segregation of sex chromosomes. $X=X$ chromosome, $X s=X$ chromosomes, $X_{1}=X_{1}$ chromosome, $X_{2}=X_{2}$ chromosome, $Y=Y$ chromosome. $\mathbf{a}$ Pholcus kindia $\left(X_{1} X_{2} Y\right)$, early diplotene. $Y$ chromosome highly condensed. In contrast, $X$ chromosomes almost decondensed; $\mathbf{b}$ Hoplopholcus cecconii $\left(X_{1} X_{2} 0\right)$, late anaphase $I . X$ chromosomes are arranged in parallel and less condensed than the other chromosomes. Moreover, their segregation and separation of their chromatids are delayed. Centromeres of sex chromosomes are formed by a prominent knob; c Cantikus sabah (X0), diplotene. Sex chromosome forms a highly condensed body; d Hoplopholcus forskali $\left(\mathrm{X}_{1} \mathrm{X}_{2} \mathrm{O}\right)$, plate formed by fusion of 1) two sister late prometaphases II (left) and 2) two sister early prometaphases II (right). In contrast to autosomes, sex chromosomes differ considerably by condensation in early and late prometaphase II. They are almost decondensed during early prometaphase II (right); e Holocneminus sp. (X0), plate formed by two sister prometaphases II, sex chromosome forms a highly condensed body; $\mathbf{f}$ Micropholcus fauroti (X0), two fused sister metaphases II. Sex chromosome shows precocious division; $\mathbf{g}$ Aetana kinabalu $\left(X_{1} X_{2} Y\right)$, late metaphase II, division of $X$ chromosomes is delayed; $\mathbf{h}$ Pholcus sp. $\left(X_{1} X_{2} Y\right)$, two halfplates of anaphase II containing positively heteropycnotic $Y$ chromosome in the middle; $\mathbf{i}$ Psilochorus simoni (X0), two sister anaphases II. Segregation of $X$ chromosome delayed. This element is slightly positively heteropycnotic at right anaphase II. Bar $=10 \mu \mathrm{m}$.

Additional file 26: Fig. S21. Arteminae, Wugigarra sp., male meiosis, behaviour of sex chromosomes. $X=X$ chromosome, $Y=Y$ chromosome, $\|$ = bivalent containing two chiasmata. a Metaphase I, three bivalents include two chiasmata. Pairing of metacentric chromosomes $X$ and $Y$ is ensured by ends of their arms. b Two sister metaphases II (separated by dashed line). While $X$ chromosome is placed at the periphery of one plate, $Y$ chromosome is in middle of another plate. Note positive heteropycnosis of sex chromosomes. Bar $=10 \mu \mathrm{m}$.
Additional file 27: Fig. S22. Pholcus sp. $\left(X_{1} X_{2} Y\right)$, testes, endopolyploid nucleus. Heterochromatic body in the middle of the nucleus is formed by sex chromosomes (arrow). Inset: a standard diploid nucleus. Bar $=5 \mu \mathrm{m}$.

Additional file 28: Appendix S1. Evolution of particular chromosomes of the pholcid $X_{1} X_{2} Y$ system.

Additional file 29: Fig. S23. Arteminae, Modisiminae, and Ninetinae: characters of NORs and sex chromosomes mapped on a cladogram. Based on the most recent molecular phylogeny of Pholcidae $[20,21]$. Standard continuous lines: low bootstrapping branch support, i.e. $<70 \%$; thick continuous lines: modest to full support of hypotheses, $\geq 70 \%$ [21]; discontinuous lines: taxa not included in molecular phylogeny - phylogenetic position is based on cytogenetic data (dashed lines) or unresolved (dotted lines). Number above mark: number of character; number below mark: character state. Full marks: characters without homoplasy; empty marks: homoplasic characters. In some species, data on some characters are missing (information in square brackets). The reconstruction of karyotype evolution is based on our data, except for taxa marked by the formula RX (see reference $X$ for information). See Methods (pp. 32-33) for the coding of characters.

Additional file 30: Fig. S24. Smeringopinae: karyotype characters mapped on a cladogram. Based on the most recent molecular phylogeny of Pholcidae [20, 21]. Standard continuous lines: low bootstrapping branch support, i.e. $<70 \%$; thick continuous lines: modest to full support of hypotheses, $\geq 70 \%$ [21]; discontinuous lines: taxa not included in molecular phylogeny - phylogenetic position is based on other sources [79] and/or on cytogenetic data (dashed lines) or unresolved (dotted lines). Number above mark: number of character; number below mark: character state. Full marks: characters without homoplasy; empty marks: homoplasic characters. In some species, data on some characters are missing (information in square brackets). The reconstruction of karyotype evolution is based on our data, except for taxa marked by the formula (RX) (some information taken from reference X). See Methods (pp. 32-33) for coding of the characters.

Additional file 31: Fig. S25. Pholcinae: characters of NORs and sex chromosomes mapped on a cladogram. Based on the most recent molecular phylogeny of Pholcidae $[20,21]$. Standard continuous lines: low bootstrapping branch support, i.e. < 70\%; thick continuous lines: modest to full support of hypotheses, $\geq 70 \%$ [21]; dashed lines: taxa not included in molecular phylogeny - phylogenetic position is based on cytogenetic data. Number above mark: number of character; number below mark: character state. Full marks: characters without homoplasy; empty marks: homoplasic characters. In some species, data on some characters are missing (information in square brackets). The reconstruction of karyotype evolution is based on our data, except for taxa marked by the formula RX (see reference X for information). See Methods (pp. 32-33) for coding of the characters.

Additional file 32: Table S6. Species studied, their instar, sex, collecting data and depositories. Abbreviations: $\mathrm{AM}=$ Australian Museum, Sydney, Australia (specimen KS 128687), Co. = county, Hwy = highway, $|\mathrm{s}|$. = island, $\mathrm{m}=$ male, Mts. = mountains, $\mathrm{N}=$ north, N.P. = national park, NW = northwest, $\mathrm{S}=$ south, $\mathrm{SL}=$ specimens lost or discarded, $\mathrm{sm}=$ subadult male, $\mathrm{W}=$ west, $\mathrm{ZFMK}=$ Zoological Research Museum Alexander Koenig.

\section{Acknowledgements}

Our paper is dedicated to J. Hajer (Purkyně University, Ústí nad Labem, Czech Republic), an outstanding specialist in functional morphology, ethology, life cycles, and spinning of spiders, who drew the attention of the principal authors to pholcids. We are very thankful to the relevant authorities for granting collection permits. We are also much obliged to the following colleagues that collected spiders, organized collections, prepared chromosome slides during expeditions, or determined specimens: N.M. Abdul Aziz, I. Agnarsson, S. Aharon, T. Andersen, R. Bennett, G. Binford, M. Chatzaki, C. Copley, D. Copley, P. Dolejš, M.A. Dias, R. Duncan, E. Gavish-Regev, A.R. Ghazali, A. Giupponi, A.V. Gromov, T.L. Heller, S. Henriquez, S. Huber, M. Johnston Pokorná, M. Komnenov, O. Košulič, C. Kristensen, L. Krkavcová, C. Leh Moi Ung, Y. Lubin, P. Michalik, J.A. Neethling, J.G. Palacios Vargas, S. Pekár, A. Pérez-González, R. Raven, M. Řezáč, J. 
Ricetti, C. Rheims, K. Rosová, D.M. Rowell, N. Šejgunovová, M. Sidibe, H. Smith, J. Starrett, L.Sousa Carvalho, A. Valdez-Mondragón, and R. Whyte. Finally, we are grateful to the anonymous reviewers for comments and suggestions on the manuscript.

\section{Authors' contributions}

Authors collected specimens (BAH, CRH, FS, IMAH, JK, MF, MH, MK, MP, PJ, PN), determined specimens (BAH, CRH), prepared chromosome slides (FS, IMAH, JK, $J M, M F, M H, M K, M P, P J)$, performed detection of nucleolus organizer regions by fluorescent in situ hybridization ( $F S, I M A H, M F, M P, M Z, P N$ ), analysed cytogenetic data (DS, FS, IMAH, JK, JM, MF, MH, MK, MP), made evolutionary analysis of cytogenetic data (BAH, DS, JK), and prepared manuscript (IMAH, $J K, M F, T K)$. All authors contributed to the final manuscript and approved its content. IMAH and JK are principal authors; they contributed equally to the paper. All authors read and approved the final manuscript.

\section{Funding}

Authors were supported by Czech Science Foundation (project no. 16-10298S: IMAH, JK, MF, PJ, PN, MK, TK), Grant Agency of the Charles University (project no. 92218: MF), Ministry of Education, Youth, and Sports of the Czech Republic (projects LTAUSA 19142: IMAH, JK, MF, TK; SWV 260314: IMAH, JK, MF; SW 260571/2021: MH, PJ), German Research Foundation (DFG projects HU 980/9-1, HU 980/11-1: BAH), National Research Foundation of South Africa (projects 97495 and 105318), and Chilean National Commission for Scientific and Technological Research (CONICYT) (IMAH). Fluorescence microscopy was performed in the Laboratory of Confocal and Fluorescence Microscopy, Faculty of Science, Charles University (Prague). This laboratory is co-financed by the European Regional Development Fund and the state budget of the Czech Republic, projects nos CZ.1.05/4.1.00/16.0347 and CZ.2.16/3.1.00/21515, and supported by the Czech-Biolmaging large RI project LM2015062. The authors are responsible for the design of the study, the collection, analysis and interpretation of data as well as writing the manuscript. The funding bodies were not involved in the research process.

\section{Availability of data and materials}

All supporting data are provided in the additional files. The partial 185 rDNA sequence of Dysdera erythrina was deposited in GenBank under the Acc. no. MT886274.

\section{Ethics approval and consent to participate}

Not applicable.

\section{Consent for publication}

Not applicable.

\section{Competing interests}

There are no competing interests.

\section{Author details}

'Laboratory of Arachnid Cytogenetics, Department of Genetics and Microbiology, Faculty of Science, Charles University, Viničná 5, 12844 Prague 2, Czech Republic. ${ }^{2}$ Research Team of Plant Stress Biology and Biotechnology, Division of Crop Genetics and Breeding, Crop Research Institute, Drnovská 507/73, 161 00 Prague 6, Czech Republic. ${ }^{3}$ Prague 1, Czech Republic. Invertebrate Zoology Unit, Department of Zoology, Faculty of Science, Charles University, Viničná 7, 12844 Prague 2, Czech Republic. ${ }^{5}$ Laboratory of Molecular Cytogenetics, Department of Molecular Biology and Genetics, Faculty of Science, University of South Bohemia, Branišovská 31, 37005 České Budějovice, Czech Republic. ${ }^{6}$ Laboratory of Molecular Cytogenetics, Department of Molecular Biology and Genetics, Institute of Entomology, Biology Centre CAS, Branišovská 31, 370 05 České Budějovice, Czech Republic. ${ }^{7}$ Research Group of Arachnid Systematics and Ecology, Department of Zoology and Entomology, Faculty of Natural and Agricultural Sciences, University of the Free State, P.O. Box 339, Bloemfontein 9300, Republic of South Africa. ${ }^{8}$ Arachnida Section, Alexander Koenig Zoological Research Museum, Adenauerallee 160,53113 Bonn, Germany.

Received: 16 May 2020 Accepted: 25 January 2021

Published online: 03 May 2021

\section{References}

1. Traut W, Ahola V, Smith DAS, Gordon IJ, ffrench-Constant RH. Karyotypes versus genomes: the nymphalid butterflies Melitaea cinxia, Danaus plexippus, and D. chrysippus. Cytogenet Genome Res. 2017;153:46-53.

2. Tomaszkiewicz M, Medvedev P, Makova KD. Y and W chromosome assemblies: approaches and discoveries. Trends Genet. 2017;33:266-82.

3. Deakin JE, Ezaz T. Understanding the evolution of reptile chromosomes through applications of combined cytogenetics and genomics approaches. Cytogenet Genome Res. 2019;157:7-20.

4. Schwager E, Sharma PP, Thomas C, Leite DJ, Wierschin T, Pechmann M, et al. The house spider genome reveals an ancient whole-genome duplication during arachnid evolution. BMC Biol. 2017;15:62.

5. Král J, Kořínková T, Forman M, Krkavcová L. Insights into the meiotic behavior and evolution of multiple sex chromosome systems in spiders. Cytogenet Genome Res. 2011;133:43-66.

6. Král J, Kořínková T, Krkavcová L, Musilová J, Forman M, Ávila Herrera IM, et al. Evolution of karyotype, sex chromosomes, and meiosis in mygalomorph spiders (Araneae: Mygalomorphae). Biol J Linnean Soc. 2013;2:377-408.

7. Král J. Evolution of multiple sex chromosomes in the spider genus Malthonica (Araneae: Agelenidae) indicates unique structure of the spider sex chromosome systems. Chromosome Res. 2007;15:863-79.

8. Garb JE, Sharma PP, Ayoub NA. Recent progress and prospects for advancing arachnid genomics. Curr Opin Insect Sci. 2018;25:51-7.

9. Král J, Musilová J, Štáhlavský F, Řezáč M, Akan Z, Edwards RL, et al. Evolution of the karyotype and sex chromosome systems in basal clades of araneomorph spiders (Araneae: Araneomorphae). Chromosome Res. 2006;14:859-80.

10. Wheeler WC, Coddington JA, Crowley LM, Dimitrov D, Goloboff PA, Griswold CE, et al. The spider tree of life: phylogeny of Araneae based on target-gene analyses from an extensive taxon sampling. Cladistics. 2017;33:574-616.

11. Shao L, Li S. Early Cretaceous greenhouse pumped higher taxa diversification in spiders. Mol Phylogenet Evol. 2018;127:146-55.

12. Michalik P, Kallal R, Dederichs TM, Labarque FM, Hormiga G, Giribet G, et al. Phylogenomics and genital morphology of cave raptor spiders (Araneae, Trogloraptoridae) reveal an independent origin of a flow-through female genital system. J Zool Syst Evol Res. 2019;57:737-47.

13. World Spider Catalog. Version 20.5. Natural History Museum, Bern. 2020. http://wsc.nmbe.ch. Accessed 4 Jan 2020.

14. Král J, Forman M, Kořínková T, Reyes Lerma AC, Haddad CR, Musilová J, et al. Insights into the karyotype and genome evolution of haplogyne spiders indicate a polyploid origin of lineage with holokinetic chromosomes. Sci Rep. 2019;9:3001.

15. Ávila Herrera IM, Carabajal Paladino LZ, Musilová J, Palacios Vargas JG, Forman M, Král J. Evolution of karyotype and sex chromosomes in two families of haplogyne spiders, Filistatidae and Plectreuridae. In: Martins C, Pedrosa-Harand A, Houben A, Sullivan B, Martelli L, O’Neil R. editors. 21st international chromosome conference, Foz do Iguaçu, Brazil. Cytogenet Genome Res. 2016;148:104.

16. Paula-Neto E, Cella DM, Araujo D, Brescovit AD, Schneider MC. Comparative cytogenetic analysis among filistatid spiders (Araneomorphae: Haplogynae). J Arachnol. 2017;45:123-8.

17. Araujo D, Schneider MC, Zacaro AA, de Oliveira EG, Martins R, Brescovit AD. Venomous Loxosceles species (Araneae, Haplogynae, Sicariidae) from Brazil: $2 n \sigma^{\star}=23$ and $X_{1} X_{2} Y$ sex chromosome system as shared characteristics. Zool Sci. 2020;37:128-39.

18. Kořínková T, Král J. Karyotypes, sex chromosomes, and meiotic division in spiders. In: Nentwig W, editor. Spider ecophysiology. Berlin: Springer; 2013. p. 159-72.

19. McStay B. Nucleolar organizer regions: genomic "dark matter" requiring illumination. Genes Dev. 2016;30:1598-610.

20. Eberle J, Dimitrov D, Valdez-Mondragón A, Huber BA. Microhabitat change drives diversification in pholcid spiders. BMC Evol Biol. 2018;18:141.

21. Huber BA, Eberle J, Dimitrov D. The phylogeny of pholcid spiders: a critical evaluation of relationships suggested by molecular data (Araneae, Pholcidae). ZooKeys. 2018;789:51-101. 
22. Huber BA. Phylogeny and classification of Pholcidae (Araneae): an update. J Arachnol. 2011;39:211-22.

23. Huber BA. Pholcidae. In: Roig-Juñent S, Claps LE, Morrone JJ, editors. Biodiversidad de Artrópodos Argentinos, vol. 3. Buenos Aires: Sociedad Entomológica Argentina; 2014. p. 131-40.

24. Araujo D, Schneider MC, Paula-Neto E, Cella DM. The spider cytogenetic database. 2020. http://www.arthropodacytogenetics.bio.br/spiderdata base. Accessed 30 Jan 2020.

25. Lomazi RL, Araujo D, Carvalho LS, Schneider MC. Small pholcids (Araneae: Synspermiata) with big surprises: the lowest diploid number in spiders with monocentric chromosomes. J Arachnol. 2018;46:45-9.

26. Araujo D, Brescovit A, Rheims C, Cella D. Chromosomal data of two pholcids (Araneae, Haplogynae): a new diploid number and the first cytogenetical record for the New World clade. J Arachnol. 2005;2:591-6.

27. Ramalho M, Araujo D, Schneider M, Brescovit A, Cella D. Mesabolivar brasiliensis (Moenkhaus 1898) and Mesabolivar cyaneotaeniatus (Keyserling 1891) (Araneomorphae, Pholcidae): close relationship reinforced by cytogenetic analyses. J Arachnol. 2008;36:453-6.

28. Bole-Gowda B. A study of the chromosomes during meiosis in twentytwo species of Indian spiders. Proc Zool Soc Bengal. 1958;2:69-108.

29. Srivastava M, Shukla S. Chromosome number and sex-determining mechanism in forty-seven species of Indian spiders. Chromos Inf Serv. 1986;41:23-6.

30. Oliveira RM, Jesus AC, Brescovit AD, Cella DM. Chromosomes of Crossopriza lyoni (Blackwall 1867), intraindividual numerical chromosome variation in Physocyclus globosus (Taczanowski 1874), and the distribution pattern of NORs (Araneomorphae, Haplogynae, Pholcidae). J Arachnol. 2007;35:293-306.

31. Golding AE, Paliulis LV. Karyotype, sex determination, and meiotic chromosome behavior of two pholcid (Araneomorphae, Pholcidae) spiders: implications for karyotype evolution. PLoS ONE. 2011;9:e24748.

32. Arunkumar $S$, Jayaprakash. Chromosomal studies of two spider species of Pholcidae (Aranae: Haplogynae). Int J Curr Res. 2015;2:2650-3.

33. Parida B, Sharma N. Chromosome number, sex mechanism and genome size in 27 species of Indian spiders. Chromos Inf Serv. 1987;43:11-3.

34. Sharma N, Parida BB. Study of chromosomes in spiders from Orissa. Pranikee. 1987;8:71-6.

35. Wang X, Cui S, Yang Z, Wang J, Wang Y. On karyotype of the Pholcus affinis (Araneida: Pholcidae). Acta Arachnol Sin. 1997;1:19-22.

36. Garrison NL, Rodriguez J, Agnarsson I, Coddington JA, Griswold CE, Hamilton CA, et al. Spider phylogenomics: untangling the spider tree of life. PeerJ. 2016:4:e1719.

37. Fernández R, Kalla RJ, Dimitrov D, Ballesteros JA, Arnedo M, Giribet G, et al. Phylogenomics, diversification dynamics, and comparative transcriptomics across the spider tree of life. Curr Biol. 2018;13:2190-3.

38. Suzuki S. Cytological studies in spiders. III. Studies on the chromosomes of fifty-seven species of spiders belonging to seventeen families, with general considerations on chromosomal evolution. J Sci Hiroshima Univ Ser B. 1954;2:23-136.

39. Sharma S, Ramakrishna S. Cytological studies on three species of Indian spiders. Int J Adv Sci Res Manag. 2019;4:1-6.

40. Rieseberg LH. Chromosomal rearrangements and speciation. Trends Ecol Evol. 2001;7:351-8.

41. Ayala F, Coluzzi M. Chromosome speciation: humans, Drosophila, and mosquitoes. Proc Natl Acad Sci USA. 2005;1:6535-42.

42. Silva D. Estudio cariotípico de Loxosceles laeta (Araneae: Loxoscelidae) Rev Perú Ent. 1988;31:9-12.

43. Silva RW, Klisiowicz DR, Cella DM, Mangili OC, Sbalqueiro IJ. Differential distribution of constitutive heterochromatin in two species of brown spider: Loxosceles intermedia and L. laeta (Araneae, Sicariidae), from the metropolitan region of Curitiba, PR (Brazil). Acta Biol Par Curitiba. 2002;31:123-36.

44. Selden PA, Penney D. Fossil spiders. Biol Rev Camb Philos Soc. 2009:85:171-206.

45. Araujo D, Schneider MC, Paula-Neto E, Cella DM. Sex chromosomes and meiosis in spiders: a review. In: Swan A, editor. Meiosis: molecular mechanisms and cytogenetic diversity. Rijeka: InTech; 2012. p. 87-108.

46. Řezáč M, Král J, Musilová J, Pekár S. Unusual karyotype diversity in the European spiders of the genus Atypus (Araneae: Atypidae). Hereditas. 2006;143:123-9.
47. Souza LB, Brescovit AD, Araujo DS. A new species of Synotaxus and the first chromosomal study on Synotaxidae, presenting a rare $X Y$ sex chromosome system in spiders (Araneae, Araneoidea). Zootaxa. 2017;4303:140-50.

48. Araujo D, Oliveira EG, Giroti AM, Mattos VF, Paula-Neto E, Brescovit AD, et al. Comparative cytogenetics of seven Ctenidae species (Araneae). Zool Sci. 2014;31:83-8.

49. Datta SN, Chatterjee K. Chromosomes and sex determination in 13 araneid spiders of North-Eastern India. Genetica. 1988;76:91-9.

50. Mahadevaiah SK, Lovell-Badge R, Burgoyne PS. Tdy-negative XY, XXY and $X Y Y$ female mice: breeding data and synaptonemal complex analysis. J Reprod Fertil. 1993;97:151-60.

51. Maddison WP. XXXY sex chromosomes in males of the jumping spider genus Pellenes (Araneae: Salticidae). Chromosoma. 1982;5:23-37.

52. Rowell DM. Complex sex-linked fusion heterozygosity in the Australian huntsman spider Delena cancerides (Araneae: Sparassidae). Chromosoma. 1985;93:169-76.

53. Maddison WP, Leduc RG. Multiple origins of sex chromosome fusions correlated with chiasma localization in Habronattus jumping spiders (Araneae: Salticidae). Evolution. 2013;67:2258-72.

54. Martin LT. Sex chromosome translocations in the evolution of reproductive isolation. Genetics. 1972;72:317-33.

55. Presgraves DC. Sex chromosomes and speciation in Drosophila. Trends Genet. 2008;24:336-43.

56. Kitano J, Ross JA, Mori S, Kume M, Jones FC, Chan YF, et al. A role for a neo-sex chromosome in stickleback speciation. Nature. 2009:461:1079-83.

57. Hooper DM, Griffith SC, Price TD. Sex chromosome inversions enforce reproductive isolation across an avian hybrid zone. Mol Ecol. 2019;28:1246-62

58. Lima TG. Higher levels of sex chromosome heteromorphism are associated with markedly stronger reproductive isolation. Nat Commun. 2014;5:4743.

59. Miller DA, Dev VG, Tantravahi R, Miller OJ. Suppression of human nucleolus organizer activity in mouse-human somatic hybrid cells. Exp Cell Res. 1976;101:235-43.

60. Dunlop JA, Penney D, Jekel D. A summary list of fossil spiders and their relatives. In: World Spider Catalog. Natural History Museum Bern. version 20.0. 2019. http://wsc.nmbe.ch. Accessed 4 Jan 2020.

61. Dimitrov D, Astrin JJ, Huber BA. Pholcid spider molecular systematics revisited, with new insights into the biogeography and the evolution of the group. Cladistics. 2013;29:132-46.

62. McKee BD, Karpen GH. Drosophila ribosomal RNA genes function as an X-Y pairing site during male meiosis. Cell. 1990;61:61-72.

63. Mandrioli M, Bizzaro D, Giusti M, Manicardi GC, Bianchi U. The role of rDNA genes in X chromosome association in the aphid Acyrthosiphon pisum. Genome. 1999;42:381-6.

64. Roy V, Monti-Dedieu L, Chaminade N, Siljak-Yakovlev S, Aulard S, Lemeunier $F$, et al. Evolution of the chromosomal location of rDNA genes in two Drosophila species subgroups: ananassae and melanogaster. Heredity. 2005;94:388-95.

65. Forman M, Nguyen P, Hula P, Král J. Sex chromosome pairing and extensive NOR polymorphism in Wadicosa fidelis (Araneae: Lycosidae). Cytogenet Genome Res. 2013;1:43-9.

66. Mittal OP. Karyological studies on the Indian spiders VI. Chromosome number and sex-determining mechanism in the family Araneidae. Res Bull Panjab Univ Sci. 1966;17:335-51.

67. Benavente R, Wettstein R. Ultrastructural characterization of the sex chromosomes during spermatogenesis of spiders having holocentric chromosomes and a long diffuse stage. Chromosoma. 1980;77:69-81.

68. John B. Meiosis. 3rd ed. Cambridge: Cambridge University Press; 1990.

69. Lukaszewski AJD, Kopecky G. Inversions of chromosome arms 4AL and $2 \mathrm{BS}$ in wheat invert the patterns of chiasma distribution. Chromosoma. 2012;121:201-8.

70. Alberti G, Weinmann C. Fine structure of spermatozoa of some labidognath spiders (Filistatidae, Segestriidae, Dysderidae, Oonopidae, Scytodidae, Pholcidae; Araneae; Arachnida) with remarks on spermiogenesis. J Morphol. 1985;185:1-35.

71. Sokolow II. Endomitotic polyploidy in testicular epithelial cells of spiders (Araneina). II. Cytology. 1967;9:257-64. (in Russian). 
72. Gregory TR, Shorthouse DP. Genome sizes of spiders. J Hered. 2003;94:285-90.

73. Hackman W. Chromosomenstudien an Araneen mit besonderer Berücksichtigung der Geschlechtschromosomen. Acta Zool Fennica. 1948:54:1-101.

74. Huber BA. Southern African pholcid spiders: revision and cladistic analysis of Quamtana gen. nov. and Spermophora Hentz (Araneae: Pholcidae), with notes on male-female covariation. Zool J Linn Soc. 2003;139:477-527.

75. Brignoli PM. Beitrag zur Kenntnis der mediterranen Pholcidae (Arachnida, Araneae). Mitt Zool Mus Berlin. 1971;47:255-67.

76. Brignoli PM. Ragni di Grecia IX. Specie nuove o interessanti delle famiglie Leptonetidae, Dysderidae, Pholcidae ed Agelenidae (Araneae). Rev Suisse Zool. 1976;83:539-78.

77. Brignoli PM. Spiders from Lebanon, V. On Hoplopholcus cecconii Kulczynski, 1908 (Pholcidae). Bull Br Arachnol Soc. 1979:4:350-2.

78. Huber BA. New World pholcid spiders (Araneae: Pholcidae): a revision at generic level. Bull Am Mus Nat Hist. 2000;254:1-348.

79. Huber BA. Revision and cladistic analysis of the Afrotropical endemic genus Smeringopus Simon, 1890 (Araneae: Pholcidae). Zootaxa. 2012;3461:1-138.

80. Dolejš P, Kořínková T, Musilová J, Opatová V, Kubcová L, Buchar J, et al. Karyotypes of central European spiders of the genera Arctosa, Tricca and Xerolycosa (Araneae: Lycosidae). Eur J Entomol. 2011;108:1-16.

81. Levan AK, Fredga K, Sandberg AA. Nomenclature for centromeric position on chromosomes. Hereditas. 1964;52:201-20.
82. Cokendolpher JC. Karyotypes of three spider species (Araneae: Pholcidae: Physocyclus). J NY Entomol Soc. 1989;97:475-8.

83. Cokendolpher JC, Brown JD. Air-dry method for studying chromosomes of insects and arachnids. Entomol News. 1985;3:114-8.

84. Galián J, Proenca SJR, Vogler AP. Evolutionary dynamics of autosomalheterosomal rearrangements in a multiple-X chromosome system of tiger beetles (Cicindelidae). BMC Evol Biol. 2007;7:158.

85. Graves JAM, Wakefield MJ, Toder R. The origin and evolution of pseudoautosomal regions of human sex chromosomes. Hum Mol Genet. 1998;7:1991-6.

86. Bačovský V, Čegan R, Šimoníková D, Hřibová E, Hobza R. The formation of sex chromosomes in Silene latifolia and S. dioica was accompanied by multiple chromosomal rearrangements. Front Plant Sci. 2020;11:205.

87. Kejnovský E, Hobza R, Čermák T, Kubát Z, Vyskot B. The role of repetitive DNA in structure and evolution of sex chromosomes in plants. Heredity. 2009;102:533-41.

88. Schartl M, Schmid M, Nanda I. Dynamics of vertebrate sex chromosome evolution: from equal size to giants and dwarfs. Chromosoma. 2016;125:553-71.

\section{Publisher's Note}

Springer Nature remains neutral with regard to jurisdictional claims in published maps and institutional affiliations.
Ready to submit your research? Choose BMC and benefit from:

- fast, convenient online submission

- thorough peer review by experienced researchers in your field

- rapid publication on acceptance

- support for research data, including large and complex data types

- gold Open Access which fosters wider collaboration and increased citations

- maximum visibility for your research: over $100 \mathrm{M}$ website views per year

At BMC, research is always in progress.

Learn more biomedcentral.com/submissions 